\title{
Energy Harvesting by means of Flow-Induced Vibrations on Aerospace Vehicles
}

\author{
Daochun $\mathrm{Li}^{1}$, Yining $\mathrm{Wu}^{1}$, Andrea Da Ronch ${ }^{2 *}$, Jinwu Xiang ${ }^{1 \dagger}$ \\ ${ }^{1}$ School of Aeronautic Science and Engineering, Beihang University, Beijing, China \\ ${ }^{2}$ Engineering and the Environment, University of Southampton, Southampton, UK
}

\begin{abstract}
This paper reviews the design, implementation, and demonstration of energy harvesting devices that exploit flow-induced vibrations as the main source of energy. Starting with a presentation of various concepts of energy harvesters that are designed to benefit from a general class of flow-induced vibrations, specific attention is then given at those technologies that may offer, today or in the near future, a potential benefit to extend the operational capabilities and to monitor critical parameters of unmanned aerial vehicles. Various phenomena characterized by flow-induced vibrations are discussed, including limit cycle oscillations of plates and wing sections, vortex-induced and galloping oscillations of bluff bodies, vortex-induced vibrations of downstream structures, and atmospheric turbulence and gusts. It was found that linear
\end{abstract}

\footnotetext{
* First Corresponding Author. Email: A.Da-Ronch@soton.ac.uk; Tel: +44(0)23-8059-4787; Postal Address: Building 13 (Tizard), room 5043, University of Southampton, Southampton, SO17 1BJ, UK.

$\dagger$ Second Corresponding Author. Email: xiangjw@buaa.edu.cn; Tel: +86(0)10-8233-8786; Postal Address: New Main Building, room D503, Beihang University, Beijing, 100083, China
} 
or linearized modelling approaches are commonly employed to support the design phase of energy harvesters. As a result, highly nonlinear and coupled phenomena that characterize flow-induced vibrations are neglected in the design process. The Authors encourage a shift in the current design paradigm: considering coupled nonlinear phenomena, and adequate modelling tools to support their analysis, from a design limitation to a design opportunity. Special emphasis is placed on identifying designs and implementations applicable to aircraft configurations. Application fields of flowinduced vibrations-based energy harvesters are discussed including power supply for wireless sensor networks and simultaneous energy harvest and control. A large body of work on energy harvesters is included in this review journal. Whereas most of the references claim direct applications to unmanned aerial vehicles, it is apparent that, in most of the cases presented, the working principles and characteristics of the energy harvesters are incompatible with any aerospace applications. Finally, the challenges that hold back the integration of energy harvesting technologies in the aerospace field are discussed.

Keywords: flow-induced vibration; energy harvesting; piezoelectric; limit cycle oscillation; vortex-induced vibration; galloping 


\section{Table of contents}

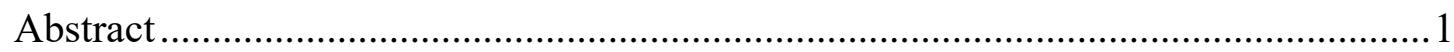

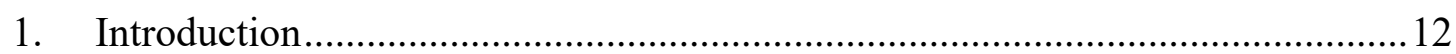

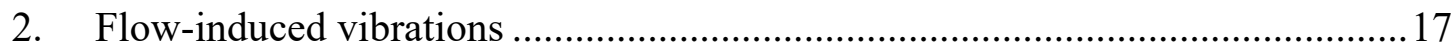

2.1 Limit cycle oscillations of plates ......................................................... 18

2.2 Galloping and vortex-induced vibrations of bluff bodies ........................... 19

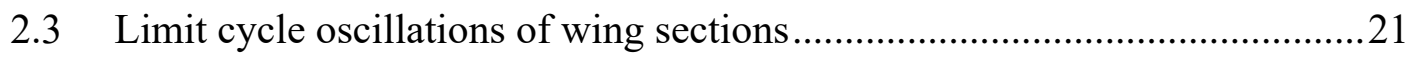

2.4 Vortex-induced vibrations generated in wake vortices .............................23

2.5 Turbulence and other variable flow-based vibrations...............................24

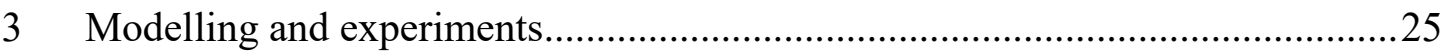

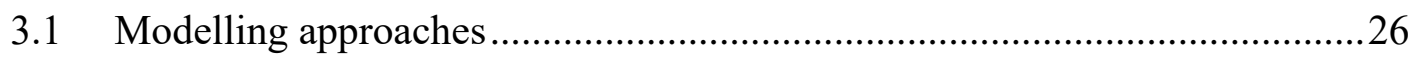

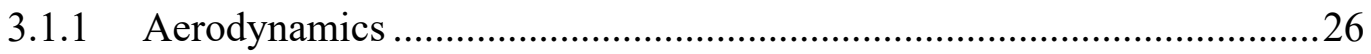

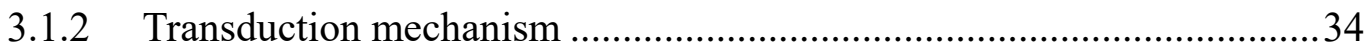

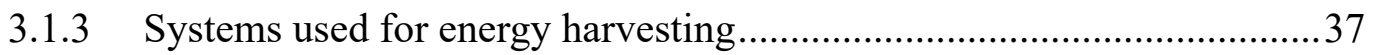

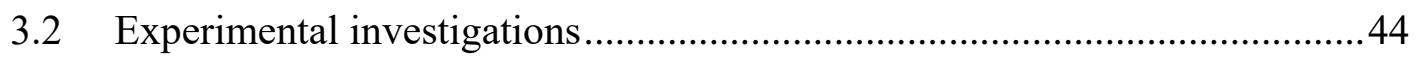

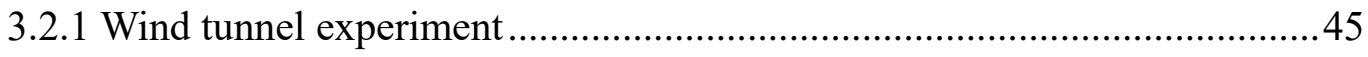

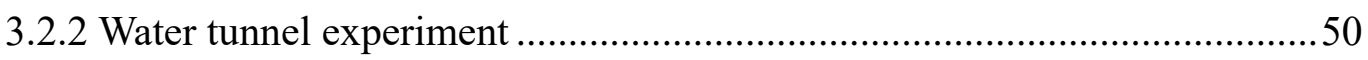

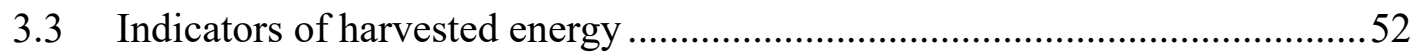

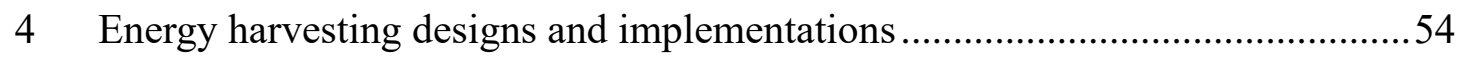


4.1 Limit cycle oscillations of plates

4.2 Galloping and vortex-induced vibrations of bluff bodies 56

4.3 Limit cycle oscillations of wing sections 61

4.4 Vortex-induced vibrations generated in wake vortices .64

4.5 Turbulence and other variable flow-based vibrations 67

4.6 In-aircraft energy harvesting implementations .68

5 Applications of energy harvesting 72

5.1 Power supply for wireless sensor networks .72

5.2 Simultaneous energy harvest and control 76

6 Summary and Challenges 80

6.1 Modelling of nonlinear coupled aero-electro-elastic model.... 81

6.2 Experimental testing.... 82

6.3 Enhancing harvested energy and performance

6.4 Scaling effects on the energy harvesting performance .83

6.5 Design and power management of host systems 84

6.6 Balance between advantages and disadvantages of energy harvesting. .84

Acknowledgement 86

References .86

Figure Captions. 
Tables 


\begin{tabular}{|c|c|}
\hline \multicolumn{2}{|r|}{ Nomenclature } \\
\hline \multicolumn{2}{|c|}{ Symbols } \\
\hline$a_{1}, a_{3}$ & coefficients to calculate the fluid force on bluff bodies \\
\hline$a$ & dimensionless location of the elastic axis \\
\hline$A_{1}, A_{2}$ & coefficients of Wagner function \\
\hline$A_{f}$ & frontal area of the harvester in operation \\
\hline$b$ & characteristic length \\
\hline$B$ & flux density at each coil segment \\
\hline$B l$ & electromagnetic coupling coefficient \\
\hline $\mathbf{B}_{\mathbf{l}}$ & electromagnetic coupling matrix \\
\hline$b_{1}, b_{2}$ & coefficients of Wagner function \\
\hline$c$ & damping coefficient \\
\hline$c l$ & sectional lift coefficient \\
\hline$c_{d}$ & sectional drag coefficient \\
\hline$c_{m}$ & sectional moment coefficient \\
\hline$c_{S}$ & nonlinear parameter due to stall \\
\hline c & elastic stiffness matrix of piezoelectric materials \\
\hline$C_{P}$ & capacitance of the piezoelectric materials \\
\hline $\mathbf{C}_{\mathbf{p}}$ & equivalent piezoelectric capacitance matrix \\
\hline $\mathbf{C}$ & damping matrix \\
\hline$d l_{\text {coil }}$ & vector of each segment of the coil \\
\hline$D$ & aerodynamic drag \\
\hline
\end{tabular}




\begin{tabular}{|c|c|}
\hline $\mathbf{D}$ & electric displacement vector \\
\hline$e$ & total flow energy \\
\hline $\mathbf{e}$ & piezoelectric stress matrix \\
\hline$E$ & harvested energy \\
\hline$E_{0}$ & total energy \\
\hline $\mathbf{E}$ & electric field vector \\
\hline $\mathbf{f}$ & external force vector \\
\hline$F_{\text {ema }}$ & electromagnetic force \\
\hline$F_{\text {emo }}$ & electromotive force \\
\hline $\mathbf{F}$ & vector of generalized aerodynamics \\
\hline$G I_{t}$ & torsional stiffness per length \\
\hline$h$ & plunge displacement \\
\hline$i$ & induced current in the coil \\
\hline$I$ & induced electric current flowing \\
\hline$I_{\alpha}$ & moment of inertia per length \\
\hline $\mathbf{I}$ & vector of induced electric current flowing \\
\hline$k$ & stiffness \\
\hline $\mathbf{K}$ & stiffness matrix \\
\hline$l$ & span length \\
\hline$L$ & aerodynamic lift \\
\hline$L_{c}$ & internal coil inductance \\
\hline $\mathbf{L}_{\mathbf{c}}$ & inherent coil inductance matrix \\
\hline
\end{tabular}




\begin{tabular}{|c|c|}
\hline$m$ & mass per length \\
\hline$m_{T}$ & total mass \\
\hline$M$ & aerodynamic moment \\
\hline$M_{b}$ & bending moment along the length \\
\hline$M_{p}$ & bending moment generated due to the transducers \\
\hline $\mathbf{M}$ & mass matrix \\
\hline $\mathbf{O}$ & zero vector \\
\hline$p$ & flow pressure \\
\hline$P$ & power output \\
\hline$P_{0}$ & power that the structure received form the flow \\
\hline$P_{f}$ & bulk mechanical power available in flow \\
\hline $\mathbf{q}$ & thermal flux vector \\
\hline$r_{1 z}, r_{2 z}, r_{3 z}$ & coefficients of ONERA model \\
\hline$R$ & applied load resistance \\
\hline$R_{c}$ & internal resistance of the inductor coil \\
\hline$S_{z 1}, S_{z 2}, S_{z 3}$ & coefficients of ONERA model \\
\hline$S$ & characteristic area \\
\hline $\mathbf{S}$ & strain vector of piezoelectric materials \\
\hline $\mathbf{T}$ & stress vector of piezoelectric materials \\
\hline$u$ & modal time coordinate \\
\hline$U$ & flow velocity \\
\hline $\mathbf{U}$ & global vector of coordinate \\
\hline
\end{tabular}




\begin{tabular}{|c|c|}
\hline $\mathbf{v}$ & flow velocity vector \\
\hline$V$ & generated voltage \\
\hline $\mathbf{V}$ & piezoelectric voltage output vector \\
\hline$x_{\alpha}$ & dimensionless eccentricity \\
\hline$Y I$ & bending stiffness per length \\
\hline$\alpha$ & pitch displacement \\
\hline$\alpha_{e f f}$ & effective angle of attack \\
\hline$\delta$ & semi-free-play \\
\hline$\varepsilon$ & ratio of the stiffness coefficients \\
\hline$\varepsilon$ & matrix of permittivity components \\
\hline$\phi$ & modal function \\
\hline$\lambda_{1}, \lambda_{2}$ & coefficients of ONERA model \\
\hline$\mu$ & energy conversion efficiency \\
\hline$\rho$ & air density \\
\hline$\Theta$ & electromechanical coupling coefficient \\
\hline $\boldsymbol{\Theta}$ & piezoelectric coefficient matrix \\
\hline$\tau$ & stress tensor \\
\hline$\tau_{\mathbf{v}}$ & viscous stress tensor \\
\hline \multicolumn{2}{|c|}{ Superscripts } \\
\hline$b$ & bending motions \\
\hline $\mathrm{E}$ & measured at a constant electric field \\
\hline$i$ & in the inductive EH circuit \\
\hline
\end{tabular}




\begin{tabular}{|c|c|}
\hline $\mathrm{S}$ & measured at a constant strain \\
\hline$t$ & torsional motions \\
\hline $\mathrm{T}$ & transposition \\
\hline . & differential with respect to the time \\
\hline ‘ & differential with respect to the length \\
\hline \multicolumn{2}{|c|}{ Subscripts } \\
\hline$a v$ & average value \\
\hline$h$ & plunge displacement \\
\hline$i$ & orders of bending modes \\
\hline$j$ & orders of torsional modes \\
\hline$\alpha$ & pitch displacement \\
\hline \multicolumn{2}{|c|}{ Acronyms } \\
\hline CFD & Computational fluid dynamics \\
\hline DAQ & data acquisition \\
\hline DOF & degree of freedom \\
\hline DNS & Direct Numerical Simulation \\
\hline $\mathrm{EH}$ & energy harvesting \\
\hline FEM & finite element method \\
\hline FIV & flow-induced vibration \\
\hline HALE & high-altitude long-endurance \\
\hline HAWT & horizontal axis wind turbine \\
\hline HB & harmonic balance \\
\hline
\end{tabular}




\begin{tabular}{|c|c|}
\hline IB & immersed boundary \\
\hline IPMC & ionic polymer metal composites \\
\hline LB & Lattice Boltzmann \\
\hline LFD & linear frequency domain \\
\hline $\mathrm{LCO}$ & limit cycle oscillation \\
\hline LDV & laser Doppler velocimetry \\
\hline MAV & micro aerial vehicle \\
\hline MPPT & maximum power point tracker \\
\hline NI & National Instruments \\
\hline ODE & ordinary differential equation \\
\hline PIV & particle image velocimetry \\
\hline RANS & Reynolds-averaged Navier-Stokes \\
\hline RMS & root mean square \\
\hline ROM & reduced order model \\
\hline UAV & unmanned aerial vehicle \\
\hline UVLM & unsteady vortex-lattice methods \\
\hline VAACT & vertical axis autorotation current turbine \\
\hline VIV & vortex-induced vibration \\
\hline VIVACE & vortex-induced vibration aquatic clean energy \\
\hline WSN & wireless sensor network \\
\hline
\end{tabular}




\section{Introduction}

One of the greatest challenges in aerospace engineering is the limited energy available during flight. This problem affects aircraft endurance and operational flight missions. Of particular interest are unmanned aerial vehicles (UAVs) and micro aerial vehicles (MAVs) which are designed to conduct intelligence, surveillance, and reconnaissance missions [1]. The availability of more energy could contribute to higher performance and extended mission profiles.

Generally, batteries are used as storage of electricity, offer a high energy density at low cost, have a low self-discharge, and provide potentially long lifetime cycles. However, there are critical aspects related with the use of batteries which hold back their use [2]. The first practical and economical penalty is the need to replace the batteries when they achieve the end of the operating life. Thereby, they are likely to be deployed in large numbers in remote areas. A second problem is linked to safety, because modern batteries may suffer from thermal runaway, ignite, and explode due to short-circuit, extreme temperatures, or inappropriate charge or discharge, not to forget poor design.

A potential technology that may replace batteries in the near future is energy harvesting (EH). EH takes advantage of ambient energy sources to generate usable electric energy based on various transduction approaches. The harvested energy, through 
appropriate transducers specific to each application, can be stored and used to recharge on-board batteries and to operate low power consumption devices, making these selfsufficient in energy supply. Various potential energy sources for EH are shown in Fig. 1 [3]. Benefits of $\mathrm{EH}$ are that: $a$ ) there is no need to replace batteries; $b$ ) there is no need of cabling; $c$ ) they are easy to retrofit infrastructures; and $d$ ) they represent a "truly fitand-forget" approach that allows reducing physical installation, replacement and maintenance costs, and time [4]. For these properties, EH are commonly used for systems that are designed to operate in remote areas with limited power supply and maintenance, and the requirement of long service time, such as electronic sensors used for structural health monitoring. Of specific interest herein is the application of $\mathrm{EH}$ in aerospace problems, generally targeting UAVs. With EH technology, future UAVs may achieve better performance: enhanced cruise range and duration time, better maintainability and viability, and larger mission payload.

Potential energy sources for aerospace applications include wind, solar radiation, and mechanical vibration, see Fig. 1. Among these EH approaches, the study on aircraft solar EH is quite mature. Table 1 summarises the power density of different EH methods [5], and it is found that solar EH through photo-voltaic conversion provides high power output density. Solar cells have been practically implemented in highaltitude long-endurance (HALE) aircraft, and being currently demonstrated on the Solar 
Impulse $^{3}$ set to travel around the world using solar energy. Developing a solar EH system to harvest sufficient energy for a sustained flight throughout the day and night is still a challenging task. This becomes more critical for smaller scale aircraft because of the lower aerodynamic performance compared to HALE configurations. Furthermore, the design of a solar EH module involves complicated trade-offs, and the expense of solar EH systems is still relatively large. Therefore, it is worthwhile to explore EH from other applicable energy sources such as piezoelectric or vibrational $\mathrm{EH}$.

Turbines using one or more flapping foils can be used as an alternative to rotary wind turbines and river, oceanic and tidal current water turbines, although industrial development is at very early stages. Such flapping foil turbines have some key potential advantages, including lower foil velocities (and hence lower noise and wildlife impact), and more effective small-scale and shallow water operation. Reference [6] presented an extensive review on the progress in flapping foil power generation in the last few decades. The effects of a number of parameters were investigated, including foil kinematics (modes, frequencies, amplitudes and time histories of motion), foil and system geometry (shape, configuration and structural flexibility), and flow physics effects (Reynolds number and turbulence, shear flows and ground effect).

Flow-induced vibrations (FIVs) are generally considered as negative phenomena since in many cases these unwanted vibrations may cause a reduction of the life span

\footnotetext{
3 The Solar Impulse project: http://www.solarimpulse.com/
} 
and structural damage. For example, in the aerospace field, aeroelastic vibrations can jeopardize the aircraft structural integrity (e.g. flutter, limit cycle oscillations, and dynamic response to atmospheric turbulence) and their performance (e.g. decreased control effectiveness and manoeuvrability, increased drag) [7-12]. A large body of work on flutter control of aeroelastic systems is available [13-19]. With structural and aerodynamic nonlinearities, aeroelastic systems may exhibit a variety of stable and unstable vibrations (e.g. chaotic motions), and efforts have been made to reduce and suppress aeroelastic vibrations. Limit cycle oscillations (LCOs) limit the amplitude of the structure into a certain range and hence avoid the structure from being destroyed by aeroelastic instability, but LCOs affect the performance of the structure and contribute to structural fatigue damage.

Flow-induced vibrations are now considered as one of the most promising potential sources of energy. The main reason is that these vibrations can be obtained from the fluid-structure interaction by utilizing energy harvesters in various natural flow conditions. In addition, fluid-induced vibrations present advantages compared to normal vibrations. For example, vibrations due to the instability phenomenon of the structures in the flow are self-excited, which makes the corresponding energy harvesters more feasible and scalable. So far, a large body of work on EH based on flow-induced vibrations is available in the open literature. Reference [20] reviewed piezoelectric wind EH designs based on galloping and vortex-induced vibrations (VIVs) of bluff bodies 
and LCOs of wing sections. The potential of active EH was emphasised, adapting the $\mathrm{EH}$ system to changes in the surrounding environment to maintain optimal performance and the highest outputs. Since 2013, a growing number of studies on EH from bluff bodies and wing sections has been reported. Apart from these EH designs based on the galloping, VIVs or aerofoil-based vibrations, there are still a large number of potential flow-induced vibration-based designs such as using the LCOs of cantilevered plates or wake galloping phenomena, which were not included in Ref. [20]. Despite the initial intentions, most studies fail to investigate $\mathrm{EH}$ devices for realistic aerospace applications. From the open literature, it is apparent that a feasibility study of EH technology, whereby the performance of a flying prototype equipped with an EH device is compared with the baseline configuration (without $\mathrm{EH}$ ), has never been reported to date. This is surprising given the ever increasing popularity of EH in the aerospace and related disciplines.

The current review paper aims at filling that gap in documenting the methods, applications, and challenges of $\mathrm{EH}$ from flow-induced vibrations based on the latest open literatures. The objectives are to: (1) review flow-induced vibrations that have been exploited for specific EH designs, and determine key aspects which benefit EH applications; (2) overview EH designs based on different types of flow-induced vibrations including LCOs of plates and wing sections, galloping and VIVs of bluff bodies, VIVs of downstream structures and variable flow or turbulence-based 
vibrations, and illustrate the key factors of parameters of each EH design to improve EH performance; and (3) review current EH applications and address the potential applications of flow-induced vibration-based EH in the aerospace field, particularly EH for UAVs.

Different types of flow-induced vibrations being exploited for EH are summarized in Section 2. Section 3 provides the fundamental modelling and experimental approaches of flow-induced vibration-based EH systems. In Section 4, various EH concepts and implementations are reviewed. The EH applications are introduced in Section 5, and the potential methods for aerospace applications are also identified. Finally, the challenges and future applications of flow-induced vibration-based EH are discussed in Section 6.

\section{Flow-induced vibrations}

This Section introduces several flow-induced vibrations which have been exploited for specific EH designs and applications. Based on different structures and vibrational mechanisms, flow-induced vibrations are overviewed including self-excited oscillations of plates, bluff bodies, and wing sections in steady flow, vibrations due to wake vortices or turbulence. The corresponding features of each flow-induced vibration are discussed from a quantitative point of view. Starting from a general treatment of the problem as experienced in several engineering fields, the most relevant phenomena in the aerospace field are discussed to provide background information for the remainder of the paper. 


\subsection{Limit cycle oscillations of plates}

Generally, for vibrations in steady flow, the mutual interaction between fluid and structure is the most commonly observed scenario where structures become unstable as the flow velocity exceeds a critical value and undergo self-excited oscillations. A typical example of flow-induced instability is the flutter of a flexible plate that is fixed at the leading edge and free at the trailing edge in an axial flow, see Fig. 2. This configuration has been extensively investigated for its rich and complex dynamics [21-25]. Plates of this kind are also known as "flapping flags" because the motion of the waving motion of flags in the wind has the same mechanism. The origin of this instability lies in a competition between the destabilizing fluid forces and the stabilizing elastic forces (related to structural stiffness). The instability of the equilibrium position occurs as the flow velocity exceeds a critical value, resulting in self-sustained LCOs. It has been demonstrated that even with only a single stream-wise edge of the plate restrained, bending tension or geometrical nonlinearities could produce LCO amplitudes of the order of the plate thickness.

For a plate that is clamped at its leading edge and free at its trailing edge, the numerical results of root mean square (RMS) dimensionless transverse amplitude and corresponding frequency of the plate LCO for increasing flow velocity are shown in Fig. 3 [25]. For increasing flow velocity, the LCO amplitude increases and the frequency decreases. This is caused by a change of the equivalent aerodynamic stiffness 
with freestream speed. As the flow velocity increases, so does the equivalent aerodynamic stiffness. Being on the right hand side of the governing equations, an increase of the equivalent aerodynamic stiffness reduces the total stiffness of the aeroelastic system. The predicted increase in the LCO amplitude and the decrease in the frequency are therefore expected.

Apart from axial flow-based designs [26], plates subject to a cross flow may also undergo LCOs. Flexible cantilevered beams or wing-like structures are sometimes referred to as "flapping leaves", see Fig. 4. The instability and LCOs of flapping leaves are due to the interaction of the flow forces with the structure's inertia and rigidity, which is the same as plates in axial flow or the LCOs of aircraft wings [27-28]. The experimental results in [29] showed that the variations of the LCO amplitude and frequency of a flapping leaf with the flow velocity have the same tendencies as those of a flapping flag.

\subsection{Galloping and vortex-induced vibrations of bluff bodies}

Generally, bluff bodies in steady flow can undergo two different types of flowinduced vibrations: VIVs and galloping oscillations. In steady flow, a bluff body generates von Kármán vortices in the wake which, in turn, create unbalanced forces on the bluff body. In one or more narrowly limited ranges of wind speed, the exciting frequency may be close enough to one of the natural frequencies of the body causing resonance. Consequently, cross-wind oscillations may occur if the body is supported by 
bending springs allowing for plunge motions. In [30], a new type of Vertical Axis Autorotation Current Turbine (VAACT) which exploits the autorotation phenomenon being the principle of operation was investigated. The turbine, which is a hinged flat plate with one degree of freedom, rotates about its vertical axis in uniform water current. The phenomenon of autorotation is a continuous rotation of a freely rotatable body in uniform flow without external sources of supplied power, produced by the vortices forming behind the blade. In a rotating plate, a large vortex is shed from the retreating (downstream) face of the rotor, whereas no similar vortex is visible from the advancing face. Consequently, such a vortex creates a hydrodynamic moment in direction of rotation which tends to continue autorotation. Therefore, an object with autorotation motion has a good potential to be considered as an energy harvester. It was found experimentally that utilizing the extra mass moment of inertia on the VAACT causes to extract the energy as an efficient turbine in very low head current.

Numerical results of transverse amplitude of the VIV of cylinder with flow velocity under different load resistances in the EH circuit are shown in Fig. 5 [31]. For each test case, resonance is obtained at a specific flow velocity, e.g., as the load resistance is $10^{3} \Omega$, the resonance speed is about $1.5 \mathrm{~m} \mathrm{~s}^{-1}$.

Galloping oscillations refer to another dynamic instability of a bluff body in a uniform flow. Unlike VIVs, galloping oscillations are somewhat similar to flutter instead of resonance. The mechanism leading to galloping oscillations is shown in Fig. 
6. This dynamic instability is activated by the formation of inner circulation flow under the two shear layers that form as the fluid moves past an oscillator's bluff body [32]. The circulation produces a negative surface pressure which causes a net lift on the body. The net lift breaks the symmetry between the shear layers on the top and bottom surfaces of the body which produces more lift. The process continues until the energy fed to the structure by the fluid balances the energy dissipated by the structure as it displaces the adjacent fluid. This results in a steady-state fixed amplitude periodic motion known as LCO. For such steady-state self-sustained oscillations to occur, the velocity of the fluid past the oscillator must exceed a certain threshold known as the cutin flow speed which represents a Hopf bifurcation in the amplitude versus flow velocity parameter's space.

The results of analytical and numerical analysis on the transverse displacement of galloping oscillations of an isosceles triangle section bluff body with flow velocity are shown in Fig. 7 [33]. The curves show a nearly quadratic increase of the amplitude with increasing flow velocity.

\subsection{Limit cycle oscillations of wing sections}

Aeroelastic flutter of aircraft wings has been observed since the early days of flight. Flutter is a self-excited destructive oscillation due to the interaction of inertial, elastic, and aerodynamic forces. Approaching the onset of flutter in an elastically supported wing, energy from a fluid flow is transferred to the aeroelastic structure, leading to 
coupled bending and torsional vibrations of the structure [34]. Below the critical flutter wind speed, a disturbance will produce structural vibrations that decay to the initial equilibrium position. Above the flutter speed, however, these oscillations will grow, leading to large deformations of the structure and flow separation until a catastrophic structural failure will occur. For the appearance of nonlinearities in the fluid or structure, stable nonlinear LCOs will emerge [14, 35-38]. Thus, the flutter speed represents a stability boundary problem where the air flow transitions from providing a positive to negative damping effect on the structure as flow speed increases, shifting the system eigenvalues into the right side of the complex plane (instability). A typical section model with plunge and pitch degrees of freedom (DOFs) is shown in Fig. 8. This model, which provides useful insights for the wing design despite its simplicity, has been used extensively in the past when the computing power was very limited [39].

The numerical results of the variations of dimensionless plunge and pitch amplitudes of the LCO of a typical wing section with flow velocity are shown in Fig. 9. The aerofoil was supported by plunge and torsional springs with hardening cubic stiffness (the coefficient of the nonlinear term was 3 ). As the flow velocity exceeds the flutter speed where the dimensionless flow velocity reaches 1.00 , bifurcation is observed and the amplitudes of pitch and plunge increase with the velocity, as shown in Fig. 9. It is worth noting that in Fig. 9 (b) the pitch rotation exceeds 10 degrees, and that the analytical and numerical results are well beyond their range of validity limited to 
small rotations. Without a physical mechanism to simulate dynamic stall, results presented may provide erroneous information. The LCO frequency for increasing flow velocity is shown in Fig. 10, and it is found to decrease linearly with the flow velocity $[37,38]$.

\subsection{Vortex-induced vibrations generated in wake vortices}

Other than vibrations induced in steady flow, forces due to variable flow conditions are the most dominant source of structural vibrations. Those variable flow conditions may appear as vortices in the wake of a bluff body.

The wake behind a cylinder in a uniform flow, in the form of von Kármán vortices shown in Fig. 11, can induce structural vibrations of a structure located downstream of the cylinder. A schematic of this is shown in Fig. 12. The mechanisms that contribute to the driving forcing were illustrated in [40]. The first mechanism is the impingement of induced flow by the passing vortices on one side of the beam, and the second is the low pressure core region of the vortices which is present at the opposite side of the beam.

Note that the VIV discussed here is different from that in Section 2.2 where a bluff body undergoes VIV due to its own shedding vortices. The current Section aims at the VIV of the downstream structures behind the bluff body generating vortices. An experimental measurement of the relationship between the amplitude and frequency of a downstream beam VIV is shown in Fig. 13 for $\mathrm{Re}=14,800$ and for a distance between the cylinder and the downstream structure equal to two times the diameter of the cylinder. It was 
found that the amplitude exhibited a dominant frequency of $48.8 \mathrm{~Hz}$, with higher frequencies multiple of the dominant frequency [40].

Wake galloping is caused by the flow interference between bluff bodies in a flow, as shown in Fig. 14. The windward cylinder is fixed in its position and sheds vortices which are then transported downstream. The vibratory motion of the downstream cylinder depends upon the particular arrangement of the two cylinders. The appearance of vibration is limited to a particular spatial range between the cylinders. It was demonstrated that if the distance between the two cylinders is larger than approximately six times the diameter of the cylinder, then the vibration does not appear [41]. The variations of the amplitude and flow velocity for different flow-induced vibrations including wake galloping are compared in Fig. 15. It can be seen that the wake galloping phenomenon appears above a critical flow speed. By comparison, this phenomenon appears for a relative wide range of flow velocity and its amplitude undergoes slow and stable change with flow velocity [41]. Unlike flutter, the amplitude is limited to a certain upper bound.

\subsection{Turbulence and other variable flow-based vibrations}

In flight, aircraft regularly encounter atmospheric turbulence. The disturbance is regarded for linear analysis as a set of component velocities superimposed on the background steady flow. The aircraft experiences rapid changes in lift and moment forces, which cause rigid and flexible dynamic responses of the entire aircraft. The 
models used for the prediction of the aircraft response have to accommodate those events that are perceived as discrete, and usually described as gusts, as well as the phenomena described as continuous turbulence. A comprehensive treatment of this problem may be found in Ref. [42].

The atmospheric turbulence signal generated numerically and filtered by von Kármán and Dryden's spectrum in the frequency domain is shown in Fig. 16 (a) [43]. The turbulence scale is $350 \mathrm{~m}$ while the RMS turbulence velocity is $0.5 \mathrm{~m} \mathrm{~s}^{-1}$. The magnitude of turbulence is relatively large at a low frequency. As the frequency increases, the magnitude decreases rapidly. Particularly, it becomes only a hundredth of the highest velocity as the frequency exceeds $10^{2} \mathrm{~Hz}$. The numerical response of a platelike wing induced by the atmospheric turbulence above is shown in Fig. 16 (b) for a low flow velocity of $0.1 \mathrm{~m} \mathrm{~s}^{-1}$ [43]. Several test cases with respect to the piezoelectric actuators installed onto the plate-like wing are considered, including the open-circuit, short-circuit, and several controlling conditions. The tip displacement decreases with increasing frequency. Three peak values are observed at 2, 10, and $16 \mathrm{~Hz}$, corresponding to the first three order modes of the plate-like wing.

\section{Modelling and experiments}

Having first reviewed the fundamental mechanisms of flow-induced vibrations, this Section summarises the methods and tests that have been performed. The first part of the Section presents the most common numerical models used, while the remainder is 
on experimental testing. The indicators used to evaluate energy output are also introduced.

\subsection{Modelling approaches}

Modelling and simulation of EH systems involve a number of physical phenomena from a variety of disciplines. Whereas these disciplines are generally not interconnected, avoiding the complications that would otherwise arise from the (nonlinear) couplings, it is apparent that this approach is inadequate to design and optimize the performance of EH systems. To build a coupled aero-electro-elastic energy harvester model, there are two main couplings needed in the modelling: the aero-elastic and the electro-elastic coupling. Several aerodynamic models are given in Section 3.1.1, including unsteady and quasi-steady methods which are used to form the aero-elastic equations. The electro-elastic coupling including the piezoelectric effect and the electromagnetic induction is introduced in Section 3.1.2. The coupled models involving the structural models are then summarised in Section 3.1.3.

\subsubsection{Aerodynamics}

Various aerodynamic models are available and these are briefly reviewed here.

\section{a. Computational fluid dynamics}

The governing equations of the fluid flow are derived from the laws of conservation of the mass, momentum, and energy. These equations are referred to as the NavierStokes equations. The resulting equations can be written as [44]: 


$$
\left\{\begin{array}{l}
\frac{\partial \rho}{\partial t}+\nabla \cdot(\rho \mathbf{v})=0 \\
\frac{\partial(\rho \mathbf{v})}{\partial t}+\nabla \cdot(\rho \mathbf{v v})=\nabla \cdot \boldsymbol{\tau}+\rho \mathbf{f} \\
\frac{\partial(\rho e)}{\partial t}+\nabla \cdot(\rho e \mathbf{v})=\rho \mathbf{f v}-\nabla \cdot \mathbf{q}+\nabla \cdot(\boldsymbol{\tau} \mathbf{v})
\end{array}\right.
$$

where $\rho$ is the air density; $\mathbf{v}$ is the flow velocity vector; $\boldsymbol{\tau}$ is the stress tensor and equal to $-p \mathbf{I}+\boldsymbol{\tau}_{\mathbf{v}}$, where $\boldsymbol{\tau}_{\mathbf{v}}$ is the viscous stress tensor and $p$ is the flow pressure; $\mathbf{f}$ is the external force vector; $e$ is the total energy of the flow; $\mathbf{q}$ is the thermal flux vector. Direct Numerical Simulation (DNS) solves directly the governing equations, but this approach has a prohibitive cost when applied to realistic geometries. On the other hand, the Reynolds-averaged Navier-Stokes (RANS) equations find their application in several industrial problems. Various turbulence models of increasing complexity (and cost) exist to close the RANS equations. The discretisation of the governing equations often is based on a finite difference, finite element, finite volume, or Lattice Boltzmann (LB) approach. To analyse flow-induced vibrations where the boundary conditions change as the structure moves, the immersed boundary (IB) method [45], body fitted grids with mesh deformation, source terms and overset grids can be employed. Computational fluid dynamics (CFD) is capable to realistically model the flow features for complex problems, but this comes at the price of high computational cost and analysis setup. To overcome these limitations, reduced order models (ROMs) have been generated to provide a compromise between accuracy of the solution and low computational costs [46-50]. 
The use of CFD has been reported in several EH designs, such as the analyses performed in $[51,52]$. These examples remain isolate, and a routine use of CFD for design and optimization of EH devices has not been reported to date. According to the literature, a "trial-and-error" approach has been a common methodology in this area.

\section{b. Unsteady potential aerodynamics}

Using an engineering approach, it is desirable for an aerodynamic model being as simple as possible yet accurate enough. Linear aerodynamics is often used in the context of aeroelastic stability analysis because small perturbations around an equilibrium state are considered. Because of the low speed characteristics, the flow can be assumed incompressible. Further, at a reasonably high Reynolds number, the viscous effects are confined to the boundary layer and wake. Finally, the primary interest in aeroelasticity is on the lifting forces. As the motion of the structure is small enough and the angle of attack remains small without the occurrence of any flow separation, it is reasonable to safely neglect viscous effects and assume an incompressible, inviscid and irrotational flow throughout the entire flow field. For these reasons, a linear unsteady potential flow model is generally sufficient in the context on flow-induced vibration-based EH systems.

\section{Strip aerodynamics}

Based on the assumptions made, the aerodynamics can be obtained in analytic forms based on Theodorsen's theory. An extension of this approach, called strip theory, adapts 
the same two-dimensional unsteady flow model for a three-dimensional system by combining section aerodynamics with a lifting line model. Limitations of strip aerodynamics include the inability to: (a) accurately describe 3D effects that strongly affect the flow features, these being included in the analysis through simple corrections; (b) account for the influence of non-slender bodies, such as the fuselage and the nacelles, although some empirical relations have been developed over the years within an industrial environment; and (c) the lack to model flow quantities in the chord-wise direction, the lifting surface being reduced to a representative 1D line running along the wing span. For an incompressible flow, the unsteady lift and moment about the elastic axis are [53]

$$
L(t)=-\pi \rho b^{2}(U \dot{\alpha}(t)+\ddot{h}(t)-b a \ddot{\alpha}(t))-2 \pi \rho U b\left(Q_{3 / 4}(0) \varphi(t)+\int_{0}^{t} \frac{\mathrm{d} Q_{3 / 4}(\tau)}{\mathrm{d} \tau} \varphi(t-\tau) \mathrm{d} \tau\right)
$$

and

$$
\begin{aligned}
M(t)= & \pi \rho b^{2}\left[b a \ddot{h}(t)-U b\left(\frac{1}{2}-a\right) \dot{\alpha}(t)-\left(\frac{1}{8}+a^{2}\right) b^{2} \ddot{\alpha}(t)\right] \\
& +2 \pi \rho U b^{2}\left(\frac{1}{2}+a\right)\left(Q_{3 / 4}(0) \varphi(t)+\int_{0}^{t} \frac{\mathrm{d} Q_{3 / 4}(\tau)}{\mathrm{d} \tau} \varphi(t-\tau) \mathrm{d} \tau\right)
\end{aligned}
$$

where $\alpha$ is the pitch displacement, positive nose up; $h$ refers to the plunge displacement, positive downward; $b$ is a characteristic length, generally taken to be the semi-chord; $U$ is the flow velocity; $a$ is the dimensionless offset from the half-chord axis of an aerofoil to its elastic axis, positive backward; $Q_{3 / 4}(\tau)=U \alpha(t)+\dot{h}(t)+b(x)(1 / 2-a) \dot{\alpha}(t)$; and $\varphi(t)$ is the Wagner function. Jones' approximation of the Wagner function is given as 


$$
\varphi(t)=1-A_{1} \mathrm{e}^{-b_{1} \frac{U}{b} \cdot t}-A_{2} \mathrm{e}^{-b_{2} \frac{U}{b} \cdot t}
$$

where the coefficients are $A_{1}=0.165, A_{2}=0.335, b_{1}=0.0445$, and $b_{2}=0.3$, respectively.

Since the assumption is of linear aerodynamics, the effects of the various influences on the aerodynamic forces and moments are added together to find the variation of the forces and moments in time for a given motion. Considering Eq. (2), it can be observed that the lift coefficient is composed of three contributions [42]. The first contribution, which includes three acceleration terms, represents the non-circulatory part of the lift coefficient. This is generally referred to as the apparent mass effect, and relates to the mass of air being accelerated by the motion of the aerofoil, perpendicular to its chord. It is worth noting that the non-circulatory part of the lift coefficient exists even when the freestream speed is zero, a fundamental mechanism behind the ability of insects to hover. The second contribution includes the effects of initial conditions on the time evolution of the lift coefficient modulated through the Wagner function. Finally, the last contribution conveys that the lift build-up for a continuous motion is synthetized as a Fourier series of simple harmonic motions at different frequencies. This integral contribution includes unsteady effects of the flow and is referred to as the circulatory part of the lift coefficient. Similar considerations are valid for the pitch moment coefficient.

\section{Unsteady vortex lattice}

The application of the unsteady vortex-lattice methods (UVLM) has also been 
reported in the design of flow-induced vibration-based EH [54-57]. The VLM solves a set of linear equations for the unknown circulation of each aerodynamic panel discretising the lifting surfaces. For cases with geometry deformations (aeroelasticity), the matrix of influence coefficients changes and must be reformed (and resolved) at every time step. Methods based on the fast multipole are generally used to speed up calculating the velocity at every point of the lattice, which includes the influence of every other wake ring plus the influence of the wing rings. The VLM captures 3D flow features attributed to geometric parameters, such as wing sweep and dihedral, but it is limited to modelling lifting surfaces. Although attempts to model the fuselage and nacelle with aerodynamic panels exist, these are widely unorthodox and require finetuning. The VLM is often corrected with the Prandtl-Glauert compressibility correction to extend the range of predictions for compressible flows.

\section{Dynamic stall model}

For wings undergoing large amplitude motions, the aerodynamic forces and moments must be calculated using a model that accounts for the nonlinear behaviour of the aerodynamics at high angles of attack. One such model, which has been extensively applied to aeroelastic flutter analysis in the literature, is the ONERA dynamic stall model [27]. This semi-empirical model describes the aerodynamic loads in pre- and post-stall conditions as [58] 


$$
\left\{\begin{array}{l}
c_{z}(t)=c_{z a}(t)+c_{z b}(t) \\
c_{z a}(t)=t_{\tau} s_{z 1} \dot{\alpha}_{e f f}(t)+t_{\tau}^{2} s_{z 2} \ddot{\alpha}(t)+t_{\tau} s_{z 3} \dot{\alpha}(t)+c_{z \gamma} \\
t_{\tau} \dot{c}_{z \gamma}(t)+\lambda_{1} c_{z \gamma}(t)=\lambda_{1} a_{o z}\left(\alpha_{e f f}(t)+t_{\tau} \dot{\alpha}(t)\right)+\lambda_{2} a_{o z}\left(t_{\tau} \dot{\alpha}_{e f f}(t)+t_{\tau}^{2} \ddot{\alpha}(t)\right) \\
t_{\tau}^{2} \ddot{c}_{z b}(t)+t_{\tau} r_{1 z} \dot{c}_{z b}(t)+r_{2 z} c_{z b}(t)=-r_{2 z} \Delta c_{z}-t_{\tau} r_{3 z} \frac{\partial \Delta c_{z}}{\partial \alpha_{e f f}} \dot{\alpha}_{e f f}(t)
\end{array}\right.
$$

where the subscripts $z$ can be $l, m$ and $d$ (refer to lift, moment, and drag, respectively); the coefficients $s_{z 1}, s_{z 2}, s_{z 3}, r_{1 z}, r_{2 z}, r_{3 z}, \lambda_{1}$, and $\lambda_{2}$ are empirically determined constants; $t_{\tau}=b / U$, and the over dot indicates differentiation with respect to time.

The nonlinearities of unsteady aerodynamics can also be represented in the form of nonlinear functions for structures different from wing sections. For example, during the calculation of EH based on an H-shape structure, the self-excited lift and moment were nonlinear functions of the vertical and torsional displacements and their derivatives [59].

Unsteady flow problems may involve periodic oscillations, allowing the use of Fourier-based methods. Typical Fourier-based methods such as linear frequency domain (LFD) and (nonlinear) harmonic balance (HB) can reduce the computational time significantly due to direct calculation of the periodic state rather than computing the transient response $[60,61]$.

\section{c. Quasi-steady aerodynamics}

For particular flow-induced vibration problems, e.g. galloping, the oscillations of the body are characterised by a timescale much larger than the characteristic timescale of the flow, and a quasi-steady hypothesis for the aerodynamics is often justified. With 
this assumption, the aerodynamics can be further simplified neglecting unsteady terms. The linear quasi-steady lift force $L$ and drag force $D$ per unit length on a wing section are given by [62]

$$
\left\{\begin{array}{l}
L=\frac{1}{2} \rho U^{2} b c_{l}(\alpha(t)) \\
D=\frac{1}{2} \rho U^{2} b c_{d}(\alpha(t))
\end{array}\right.
$$

where $c_{l}, c_{d}$ are the sectional lift and drag coefficients, respectively. The quasi-steady aerodynamic loads with a stall model are written as [63]

$$
\left\{\begin{array}{l}
L=\frac{1}{2} \rho U^{2} b c_{l}\left(\alpha_{e f f}(t)-c_{s} \alpha_{e f f}^{3}(t)\right) \\
D=\frac{1}{2} \rho U^{2} b c_{d}\left(\alpha_{e f f}(t)-c_{s} \alpha_{e f f}^{3}(t)\right)
\end{array}\right.
$$

where $c_{s}$ is a nonlinear parameter associated with stall. The effective angle of attack $\alpha_{\text {eff }}$ due to the instantaneous motion of the aerofoil is expressed as

$$
\alpha_{e f f}(t)=\frac{Q_{3 / 4}(t)}{U}
$$

For transverse galloping oscillations, a cubic polynomial form was employed to approximate the vertical fluid force coefficient $[32,64]$

$$
L=\frac{1}{2} \rho U^{2} S\left(a_{1} \frac{\dot{h}(t)}{U}-a_{3}\left(\frac{\dot{h}(t)}{U}\right)^{3}\right)
$$

where $S$ is characteristic area, e.g. the cross-section area of the bluff body, and $a_{1}, a_{3}$ are coefficients. 


\subsubsection{Transduction mechanism}

\section{a. Piezoelectricity}

The direct piezoelectric effect makes piezoelectric materials capable of being used as harvesters [65-68]. This effect is an electromechanical phenomenon that occurs in piezoelectric materials when a coupling of electrical and mechanical states occurs due to an applied mechanical stress. On the other hand, if an electrical differential is supplied to the piezoelectric, then the system will respond with a mechanical stress in the form of a material deflection. Two practical coupling modes exist in piezoelectric materials: $31-$ mode, and 33-mode. In the 33-mode, the electronic voltage and mechanical stress act in the same direction as shown in Fig. 17 (a), while in the 31-mode the voltage acts in direction 3 and the mechanical stress acts in direction 1 as shown in Fig. 17 (b). In the 31-mode, a force is applied in the direction perpendicular to the poling direction, an example of which is a bending beam that is poled on its top and bottom surfaces. In the 33-mode, a force is applied in the same direction as the poling direction, such as the compression of a piezoelectric block that is poled on its top and bottom surfaces.

Conventionally, the 31-mode has been the most commonly used coupling mode though the 31-mode yields a lower coupling coefficient than the 33-mode [68]. For a very low pressure source and limited device size, the 31-mode conversion may be more suitable for EH, since larger strains can be produced with smaller input forces [66].

The advantages of piezoelectric EH are the high energy output density, low cost, 
long service lifetime, and compact electromechanical structure. Common piezoelectric transducers are shown in Fig. 18.

The constitutive relations of piezoelectric materials can be represented by [53]

$$
\left\{\begin{array}{l}
\mathbf{T}=\mathbf{c}^{\mathrm{E}} \mathbf{S}-\mathbf{e}^{\mathrm{T}} \mathbf{E} \\
\mathbf{D}=\mathbf{e S}+\boldsymbol{\varepsilon}^{\mathrm{S}} \mathbf{E}
\end{array}\right.
$$

where $\mathbf{T}, \mathbf{D}, \mathbf{E}$, and $\mathbf{S}$ are, respectively, the stress vector, electric displacement vector, electric field vector, and strain vector; $\mathbf{c}, \mathbf{e}$, and $\boldsymbol{\varepsilon}$ are, respectively, the elastic stiffness matrix, piezoelectric stress matrix, and matrix of permittivity components. The superscripts " $\mathrm{E}$ " and "S" are used to indicate that the corresponding parameter is measured at a constant electric field or at a constant strain, respectively; the superscript "T" indicates transposition. Note that this paper focuses on EH analysis based on linear piezoelectric constitutive relations, although piezoelectric nonlinearities need to be considered in specific cases [69].

The general principle for conversion of mechanical low frequency AC stress into electrical energy using a piezoelectric transducer is shown schematically in Fig. 19. There are three primary steps in power generation: (a) trapping the mechanical AC stress from available source; (b) converting the mechanical energy into electrical energy with the piezoelectric transducer; and (c) processing and storing the generated electrical energy using a rectifier and DC-DC converter circuit [67].

\section{b. Electromagnetic induction}

While piezoelectric transduction is a convenient method to extract energy from 
structural deformations, electromagnetic induction has particular advantages in $\mathrm{EH}$ from flow-induced vibrations, and has the ability to extract kinetic energy from relative motions via coil-magnet arrangements based on Faraday's law. As an example, the schematic of EH based on a bluff body which is linked to an electromagnetic generator in cross-flow [70] is shown in Fig. 20 (a). The magnet is attached onto the plunge DOF while the coil and external circuit are fixed on the base. An experimental setup of an electromagnetic inductor energy harvester device is shown in Fig. 20 (b) [71].

More efficient designs of the electromagnetic EH may employ multiple magnets and coils. A representative 2 DOF magneto-mechanical EH is shown in Fig. 21 (a). As the harvester is attached to a host structure which is undergoing vibrations in the direction of the longitudinal axis of the harvester, the repulsive forces generated by the two magnets act as excitation forces in combination with the elastic forces of the two springs. Compared to single DOF system that has only one resonance frequency, this arrangement allows extending the frequency bandwidth of the system when the excitation is not deterministic such as turbulence and hence improves the applicability [72]. The prototypes for this concept are shown in Fig. 21 (b).

The electromotive force $F_{\text {emo }}$ which is produced on the terminals of the coil with time constant electromagnetic field equals to

$$
F_{\text {emo }}=\oint(\dot{h} \times B) \mathrm{d} l_{\text {coil }}
$$

where $\dot{h}$ refers to the relative velocity of the coil and the magnet. The vector of each 
segment of the coil is denoted by $d l_{\text {coil }}$ and $B$ is the flux density at each coil segment. It is also assumed that the coil does not experience large changes in the magnetic field during the motion due to the small displacement of the magnet. Therefore, the relationship between the amplitude of the velocity and the electromotive force can be considered linear

$$
F_{\text {emo }}=B_{a v} l_{c o i l} \dot{h}=B_{l} \dot{h}
$$

and the electromagnetic force $F_{\text {ema }}$ acting on the bluff body can be given by:

$$
F_{\text {ema }}=-B_{a v} l_{c o i l} i=-B_{l} i
$$

where $i$ is the induced current in the coil, the proportionality constant $B_{l}$ is called the electromagnetic coupling coefficient, and the subscript " $a v$ " indicates average value. Consequently, the equations of motion that couple the aeroelastic system and the electromagnetic transducer with consideration of an electrical load resistance in the electrical circuit can be obtained [70]. The electromagnetic generator can be modelled by an equivalent electrical circuit where power is dissipated at an electrical load resistance [73]. The modelling can be generalized when there are multiple magnets and coils, such as the energy harvester in [74] that consisted of three magnets in which one magnet floated between two fixed magnets.

\subsubsection{Systems used for energy harvesting}

This Section reviews common structural components that have been used for EH. It will be shown that the structural models range from a single DOF system to a continuous representation for beams and plates. The Galerkin method and the finite 
element method (FEM) are widely used to create a mathematical model of a continuous structural system. The former uses the modal functions of the structure as base functions to obtain the equations in terms of generalized coordinates. The latter involves: (1) dividing the structure into a number of discrete elements, with each element represented by a set of equations of motion with respect to the displacements of each node; and (2) systematically assembling the element equations into a global system of equations for the final analysis. The FEM has virtually no limitations about the complexity of the geometry and the corresponding boundary conditions.

A generalized form of the coupled aero-electro-elastic flow-induced vibration-based EH model (incorporating both piezoelectricity and electromagnetic induction) is

$$
\left\{\begin{array}{l}
\mathbf{M} \ddot{\mathbf{U}}+\mathbf{C} \dot{\mathbf{U}}+\mathbf{K U}+\mathbf{F}_{\mathbf{n}}(\mathbf{U}, \dot{\mathbf{U}})-\mathbf{\Theta V}-\mathbf{B}_{\mathbf{l}} \mathbf{I}=\mathbf{F} \\
\mathbf{C}_{\mathbf{p}} \dot{\mathbf{V}}+\frac{\mathbf{V}}{R}+\boldsymbol{\Theta}^{\mathrm{T}} \dot{\mathbf{U}}=\mathbf{O} \\
\mathbf{L}_{\mathbf{c}} \dot{\mathbf{I}}+\left(R_{c}+R^{i}\right) \mathbf{I}+\mathbf{B}_{\mathbf{l}}^{\mathrm{T}} \dot{\mathbf{U}}=\mathbf{O}
\end{array}\right.
$$

where $\mathbf{M}, \mathbf{K}$ and $\mathbf{C}$ are the mass matrix, stiffness matrix, and damping matrix, respectively; $\mathbf{F}_{\mathbf{n}}$ refers to the nonlinear terms; $\mathbf{F}$ is the vector of generalized aerodynamics, which is obtained using the methods summarised in Section 3.1.1; $\mathbf{U}$ is the vector describing the structural states; $\boldsymbol{\Theta}$ is the piezoelectric coefficient matrix; $\mathbf{V}$ is the piezoelectric voltage output vector; $\mathbf{C}_{\mathbf{p}}$ is the equivalent piezoelectric capacitance matrix; $R$ is the applied load resistance; $\mathbf{B}_{\mathbf{I}}$ is the electromagnetic coupling matrix, $\mathbf{I}$ is the vector of induced electric current flowing to $R^{i}$, where $R^{i}$ is the load resistance in the inductive circuit; $\mathbf{L}_{\mathbf{c}}$ is the inherent coil inductance matrix; $R_{c}$ is the internal resistance 
of the inductor coil; and $\mathbf{O}$ represents the zero vector. The first equation in Eq. (14) describes the dynamics of the system. The second and third equations are for the piezoelectric and magnetic transducers, respectively. The three sets of equations are coupled through the electromechanical terms.

\section{a. Beams and plates}

Beams and plates are widely used in flow-induced vibration-based EH because they are scalable in size and can easily accommodate various types of transducers. Typically, linear models are used. Under some conditions of high aspect ratio and similar width-toheight ratio, plates can be modelled as beams. For example, for the flexible plate shown in Fig. 22, the span of the flexible plate is assumed to be much larger than its typical chord-wise length scale, and the thickness smaller than the length and width.

Deformations are expected to be similar to a beam-like model [75]. As the structure is inextensible and clamped at its leading edge, based on the Euler-Bernoulli beam theory, the equation of bending motion can be represented as [53]

$$
m \ddot{w}-M_{b} "=L
$$

where $m$ is the mass per unit length; the prime symbol ( $\left.{ }^{\prime}\right)$ is used to denote a spatial differentiation; and $M_{b}$ is the bending moment along the span including the contribution of the piezoelectric materials, and can be further expressed as

$$
M_{b}=-Y I w^{\prime \prime}-M_{p}
$$

where $Y I$ is the bending stiffness per unit length; $M_{p}$ is the bending moment generated from the transducers. Once the structure is exposed to cross flow, the torsional motion 
must be taken into account. Considering the existence of an eccentricity, the equations of motion become

$$
\left\{\begin{array}{l}
m \ddot{w}+m x_{\alpha} b \ddot{\theta}+Y I w^{\prime \prime \prime}+M_{p}^{\prime \prime}=-L \\
m x_{\alpha} b \ddot{w}+I_{\alpha} \ddot{\theta}-G I_{t} \theta^{\prime \prime}=M
\end{array}\right.
$$

where $I_{\alpha}$ is the moment of inertia per unit length; $G I_{t}$ is the torsional stiffness per unit length; $x_{\alpha}$ is the dimensionless eccentricity, i.e. the offset from the elastic axis to the centre of gravity axis; and $M$ refers to the aerodynamic moment along the elastic axis. Using Galerkin procedure to discretise the system, the deflection and torsional deformation are assumed to be

$$
\left\{\begin{array}{l}
w=\sum_{i} \phi_{i}^{b} u_{i}^{b} \\
\theta=\sum_{j} \phi_{j}^{t} u_{j}^{t}
\end{array}\right.
$$

where $\phi$ denotes the modal function of the beam, and the superscripts $b$ and $t$ indicate, respectively, bending and torsional motions; subscripts $i$ and $j$ indicate the orders of bending and torsional modes, respectively; and $u$ refers to the modal time coordinate corresponding to $\phi$. Substituting Eq. (18) into Eq. (17) gives the equations in discretized form

$$
\left\{\begin{array}{l}
M_{i}^{b} \ddot{u}_{i}^{b}+\sum_{j} M_{i j}^{b} \ddot{u}_{j}^{t}+K_{i}^{b} u_{i}^{b}-\sum_{k} \Theta_{i k} V_{i}=F_{i}^{b} \\
\sum_{i} M_{j i}^{t} \ddot{u}_{i}^{b}+M_{j}^{t} \ddot{u}_{j}^{t}+K_{j}^{t} u_{j}^{t}=F_{j}^{t}
\end{array}\right.
$$

where the subscript $k$ refers to the sequence of the transducers; $V$ is the generated voltage; and $\Theta$ is the electromechanical coupling coefficient. The coefficients, which constitute the matrices of Eq. (14), are obtained from $M_{i}^{b}=\int m\left(\phi_{i}^{b}\right)^{2} d x$, 


$$
\begin{aligned}
& M_{i j}^{b}=\int m x_{\alpha} b \phi_{i}^{b} \phi_{j}^{t} d x, K_{i}^{b}=\int Y I\left(\phi_{i}^{b}\right)^{\prime} d x, F_{i}^{b}=\int L \phi_{i}^{b} d x, \quad M_{j i}^{t}=\int m x_{\alpha} b \phi_{i}^{b} \phi_{j}^{t} d x, \\
& M_{j}^{t}=\int I_{\alpha}\left(x_{\alpha} b\right)^{2}\left(\phi_{j}^{t}\right)^{2} d x, K_{j}^{t}=\int G I_{t}\left(\phi_{j}^{t \prime}\right)^{2} d x, \quad F_{a j}^{t}(t)=\int M \phi_{j}^{t} d x .
\end{aligned}
$$

For large deformations, the nonlinear Euler-Bernoulli beam model is needed.

Although the beam can undergo large deformations, the strains are assumed small, so that the linear Hooke's law is used to relate the strains to the stresses [52].

The classic plate theory is generally employed to form the linear plate-based EH system. Reference [76] presented an electromechanical FEM plate model for predicting the electrical power output of piezoelectric EH plates. The FEM model was derived based on the Kirchhoff plate assumptions because typical piezoelectric energy harvesters are thin components.

\section{b. Bluff bodies}

For the VIV or galloping oscillation-based energy harvesters shown in Fig. 23, the single DOF coupled model can be developed to simulate the electro-fluid-structural coupling behaviour. The governing equations are written as [77, 78]

$$
\left\{\begin{array}{c}
m_{T} \ddot{h}+c \dot{h}+k h-\Theta V=-L \\
\frac{V}{R}+C_{p} \dot{V}+\Theta \dot{h}=0
\end{array}\right.
$$

where $m_{T}$ accounts for both the structure and the fixture mass in the experiments connecting the plunge springs, $c$ and $k$ are the damping coefficient and the stiffness of the harvester, respectively; $C_{P}$ is the total capacitance of the piezoelectric sheets in parallel connection. The aerodynamic force $L$ can be calculated based on quasi-steady 
assumption shown in Eq. (9).

When the deformation of the beams that support the bluff bodies are taken into account, the galloping-based harvesters could be modelled as one or two EulerBernoulli beams attached to a tip mass and discretized using the Galerkin procedure [79-81]. The discretization of the piezo-aero-elastic EH model carried out in Ref. [82] used the exact mode shapes of the structure. In [59], the governing equations of motion for an H-shape beam were obtained for a rigid body motion with only two modes of vertical and torsional vibrations.

Zhao et al $[83,84]$ proposed a modelling approach to evaluate the performance of galloping-based energy harvester based on equivalent circuit representation, which could tackle various interface circuits and nonlinear aerodynamic forces. The mechanical parameters and piezoelectric coupling in the system were represented by standard linear electronic components and an ideal transformer, respectively. The aerodynamic force was represented by a user-defined electronic component with a nonlinear transfer function. Based on the equivalent circuit representation, the entire energy harvester system was modelled in a circuit simulator for system-level simulation [83].

\section{c. Wings and wing sections}

A typical schematic of a 2 DOF aerofoil is shown in Fig. 8. The piezo-aero-elastic equations including a piezoelectric patch operating on the plunge DOF and with a 
resistive load in the electrical domain are [85]

$$
\left\{\begin{array}{l}
m_{T} \ddot{h}+m x_{\alpha} b \ddot{\alpha}+c_{h} \dot{h}+k_{h} h-\frac{\Theta V}{l}=-L \\
m x_{\alpha} b \ddot{h}+I_{\alpha} \ddot{\alpha}+c_{\alpha} \dot{\alpha}+k_{\alpha} \alpha=M \\
C_{p} \dot{V}+\frac{V}{R}+\Theta \dot{h}=0
\end{array}\right.
$$

where $l$ is the span of the wing section. When both piezoelectric transduction and electromagnetic induction are considered in the plunge DOF of an aerofoil $[86,87]$, the aero-electro-elastic coupled equations governing the dynamics of the hybrid piezoelectric-inductive flow energy harvester (shown in Fig. 24) become

$$
\left\{\begin{array}{l}
m_{T} \ddot{h}+m x_{\alpha} b \ddot{\alpha}+c_{h} \dot{h}+k_{h} h-\frac{\Theta V}{l}-\frac{B_{l} I}{l}=-L \\
m x_{\alpha} b \ddot{h}+I_{\alpha} \ddot{\alpha}+c_{\alpha} \dot{\alpha}+k_{\alpha} \alpha=M \\
C_{p} \dot{V}+\frac{V}{R}+\Theta \dot{h}=0 \\
L_{c} \dot{I}+\left(R_{c}+R^{i}\right) I+B_{l} \dot{h}=0
\end{array}\right.
$$

where $B_{l}$ is the electromagnetic coupling, $I$ is the induced electric current flowing to $R^{i}$, $R^{i}$ is the load resistance in the inductive EH circuit, $L_{c}$ is the internal (or inherent) coil inductance, $R_{c}$ is the internal resistance of the inductor coil. In [87], the dynamics of the control surface was taken into account so that an additional equation was introduced to Eq. (22).

When structural nonlinearity exists in the pitch or plunge DOF, a specific expression for the elastic terms will be used. If a free-play nonlinearity exists in the pitch, the nonlinear restoring term can be written as [88] 


$$
k(\alpha) \alpha=\left\{\begin{array}{cc}
k_{\alpha}(\alpha-\delta) & (\alpha>\delta) \\
0 & (-\delta<\alpha<\delta) \\
k_{\alpha}(\alpha+\delta) & (\alpha<-\delta)
\end{array}\right.
$$

where $\delta$ is semi-free-play. If the nonlinearity is of a cubic hardening type, then [88]

$$
k(\alpha) \alpha=k_{\alpha}\left(\alpha+\varepsilon \alpha^{3}\right)
$$

where $\varepsilon$ is the ratio of the stiffness coefficients. A number of time integration schemes exist to solve efficiently nonlinear equations that are formulated as a set of first order ordinary differential equations (ODEs). For this reason, it is common practice to recast the governing equations as first order ODEs and employ off-the-shelf numerical algorithms for the time integration.

A conclusive remark is on the limitative prediction capabilities of the numerical models employed for the EH design. The choice of the numerical models is driven by two contrasting requirements: being fast enough for design purposes, and being accurate enough for realistic predictions. The former requirement is generally dominant, with the latter accounted for using various semi-empirical corrections. The types of flow features exploited for EH are extremely challenging from a numerical point of view, and aerodynamic modelling is one of the largest sources of uncertainties.

\subsection{Experimental investigations}

Whereas the generality of numerical models is restricted from the underlying assumptions, experimental testing can provide physical insights on the system being tested. Experimental testing, however, is not suited for the preliminary design phases, as 
the testing requires the experimental model to be designed and manufactured. The type of testing, carried out either in a wind or water tunnel, is affected by kinematical constraints, and interference effects which may be difficult or impossible to remove or separate from the measurements. Rather than keeping numerical modelling and experimental testing separate, it is the authors' experience that better designs with improved performances may be achieved by an adequate combination of the two approaches.

\subsubsection{Wind tunnel experiment}

For a T-shaped piezoelectric EH device shown in Fig. 25 (a), it is relatively difficult to build a representative aeroelastic model. In order to investigate the aeroelastic response and power output of the device, an experimental setup in a wind tunnel was used in [89], see Fig. 25 (b). The dimensions of the cantilever beam are $100 \mathrm{~mm} \times$ $60 \mathrm{~mm} \times 30 \mathrm{~mm}$ (length, width, height). The thickness of the aluminium substrate was $0.2 \mathrm{~mm}$. A mass of $15.5 \mathrm{~g}$ was positioned at the beam tip. A total of six flexible PZT-5A piezoceramics M-2814-P2 were attached at the roots of both sides of the cantilever beam. The length and width of each piezoceramic were $28 \mathrm{~mm}$ and $14 \mathrm{~mm}$, respectively.

During wind tunnel tests, the wind speed was measured using a Pitot tube and a pressure transducer. The images acquired from the CCD camera were used to measure the tip displacement from the image processing technique. The output voltage was monitored on a digital multimeter, and was converted to power from the measured 
voltage and the applied external electrical resistance. The device was found to provide power for a wind speed larger than $4 \mathrm{~m} \mathrm{~s}^{-1}$ and a continuous peak electrical power output of $4.0 \mathrm{~mW}$.

A galloping-based energy harvester is shown in Fig. 26 (a). The device consists of a metal beam, two surface bonded piezoelectric layers, and a tip bluff body with a square cross section [90]. Specifically, macro-fibre composite piezoelectric sheets M8528-P2 were bonded to a steel cantilever beam using the M-bond 200 adhesive kit2. The tip bluff body made from polyurethane foam was firmly held at the tip section of the piezoelectric beam. Shown in Fig. 26 (b), the experiment was performed in a low-speed wind tunnel with the lowest wind speed of $1.9 \mathrm{~m} \mathrm{~s}^{-1}$. The test section was $0.3048 \mathrm{~m} \times$ $0.3048 \mathrm{~m} \times 0.61 \mathrm{~m}$ (height, width, length). The piezoelectric sheets were connected in parallel to form a bimorph configuration across electrical load resistances. Wind velocity was measured using a Pitot tube and an anemometer. Turbulence screens smoothen out any swirls in the incoming flow. A Keyence laser sensor was used to measure the displacement response at the location of $170 \mathrm{~mm}$ away from the clamped end. This location was selected for the best measurement without causing interference with the tip bluff body motion. To record the displacement and harvested voltage under different wind velocities and different resistive loads, a National Instruments (NI) data acquisition (DAQ) system was used. An in-house resistive voltage divider circuit was built to collect harvested voltage signals in order to meet the DAQ input voltage range 
$\pm 10 \mathrm{~V}$. The NI DAQ input impedance was larger than $16 \mathrm{M} \Omega(>100 \mathrm{G} \Omega$ in parallel with $10 \mathrm{pF}$ ) to avoid altering the effective impedance of the signal circuit. Signal postprocessing was conducted in order to extract the frequency components from time domain signals. The NI DIADEM software was used to determine the LCO amplitudes and harvested peak voltage values under different wind velocities and resistive loads. The harvester was tested under different electrical loads ranging from $10 \mathrm{k} \Omega$ to $1 \mathrm{M} \Omega$ and different wind velocities ranging from $2 \mathrm{~m} \mathrm{~s}^{-1}$ to $8 \mathrm{~m} \mathrm{~s}^{-1}$. The self-excited nature of the galloping phenomenon requires initial perturbations to grow until a steady-state response is obtained. The result showed that the voltage output and vibrational amplitude increased with increasing wind velocity if the electrical load was fixed. The maximum in the power output and in the amplitude of the dynamic response were obtained with increasing load resistance.

The interaction between an upstream aerofoil-based energy harvester and a downstream harvester is another complex problem. The difficulty arises from determining the flow features impacting on the harvester located downstream. Wind tunnel experiments were conducted in an open circuit, suction-type wind tunnel. The tunnel had a test section with $1.2 \mathrm{~m} \times 1.35 \mathrm{~m} \times 3.2 \mathrm{~m}$ (width, height, length) and turbulence intensity up to $2 \%$ [91]. Six axial fans at the tunnel outlet control the airflow in the test section while a Pitot static tube and an Omega HHF42 probe hotwire anemometer were used to monitor the wind tunnel flow velocity. A camera was 
mounted above the wind tunnel test section and was remotely operated by a tethered laptop computer. The two energy harvesters were positioned at the mid-height of the wind tunnel by a streamlined aluminium stings. The photograph of the harvesters in the wind tunnel test section is shown in Fig. 27 (a). An overhead schematic view of the smoke wire flow visualization setup was used to image the wake of the energy harvesters, as shown in Fig. 27 (b). The results showed that the wake of the windward harvester had significant effects on the vibration amplitude, frequency, and power output of the trailing devices. The wake interaction effects varied with the stream-wise and cross-stream separation distance between the harvesters. Over a defined range of separations, the trailing harvesters could extract additional energy from the wake of the upstream harvesters, causing larger oscillation amplitudes and higher power output in the trailing devices.

A rectangular wing-based $\mathrm{EH}$ system with a piezoelectric generator attached were designed and experimentally tested in [92]. The system was placed in a wind tunnel, shown in Fig. 28 (a), and consisted of a rectangular wing anchored on a shaft, which was attached in a test section of $2000 \mathrm{~mm} \times 780 \mathrm{~mm} \times 720 \mathrm{~mm}$ (length, width, height). The oncoming flow speed was varied from 0 to $35 \mathrm{~m} \mathrm{~s}^{-1}$. To reduce the mechanical friction, an air bearing was used. This was achieved by injecting compressed air with a uniform pressure of $689 \mathrm{kPa}$ to form a thin film for lubrication. In order to harvest the kinetic energy from the oncoming air flow via self-sustained rolling LCOs, a 
rectangular-shaped piezeoelectric generator was attached to the wings. Two different configurations for the placement of the generator were studied, Fig. 28 (b). One configuration was perpendicular to the oncoming flow streamline, while the second was parallel. To maximize the electrical power output from the piezoelectric generator, a tip mass was added. It was shown that the aeroelastic rolling frequency increased with the generator attached, whereas the rolling amplitude decreased. In addition, the wing with the smallest aspect ratio was found to be easiest to trigger the LCOs. The EH performance was maximized as the generator is attached in parallel with the oncoming flow streamline.

To study the effects of the free-play nonlinearity on the performance of an aerofoilbased energy harvester, an experimental setup was used [93], see Fig. 29 (a). The experiments were conducted in an open-circuit wind tunnel having a $520 \mathrm{~mm} \times 515 \mathrm{~mm}$ test section and with a minimum wind speed of $8.5 \mathrm{~m} \mathrm{~s}^{-1}$. The rigid aerofoil consisted of an aluminium rigid wing mounted vertically at the $1 / 4$ chord point from the leading edge. The shaft was connected with bearings to the support of the plunge mechanism, which was a bi-cantilever beam made of two steel leaf springs. A steel leaf torsional spring was inserted into a slot in the main shaft at the bottom of the wing section. The free end of the leaf spring was placed into a support, as in Fig. 29 (b), that allowed for free-play variations. An encoder and an accelerometer were used to measure the rotational and plunge motions, respectively. It was demonstrated that by increasing the 
free-play nonlinearity gap, the cut-in speed was reduced through a subcritical instability and energy could be harvested at low wind speeds.

\subsubsection{Water tunnel experiment}

Based on the literature, a number of EH systems have been designed for underwater operations. Water tunnels can be used in place of wind tunnels to perform measurements because techniques such as particle image velocimetry (PIV) are easier to implement in water. Also, as the flow characteristic parameters such as Reynolds number are set the same, experiments in a wind tunnel also can be conducted in water tunnel.

A water tunnel was used in [94] to study EH from an underwater flapping flag. The water tunnel had nominal dimensions $250 \mathrm{~cm} \times 15 \mathrm{~cm} \times 15 \mathrm{~cm}$, allowing the flow speed to be regulated up to about $1.2 \mathrm{~m} \mathrm{~s}^{-1}$. The water speed was measured with a laser Doppler velocimetry (LDV) system located $25 \mathrm{~cm}$ upstream of the flag. A schematic of the experimental setup is shown in Fig. 30 (a). The performance of the energy harvester was investigated for a number of fluid velocities and resistive loads. The device was immersed in the test section of a water tunnel filled with tap water and the onset and development of the flutter instability was studied for increasing velocities. The postflutter vibrations of the structure were tracked by means of image analysis, while the time trace of the voltage across the load was simultaneously acquired. A schematic of this is given in Fig. 30 (b). The results showed that matching the load resistance with the ionic polymer metal composites (IPMC) electrical characteristics maximised the 
scavenged power. The harvested power with optimal load was close to $10^{-10} \mathrm{~W}$ and was reasonably steady for mean flow speeds between 0.6 and $1.1 \mathrm{~m} \mathrm{~s}^{-1}$.

The experiments presented in [95], studying the flow over a cylinder with a hingedsplitter plate shown in Fig. 31 , were conducted in a $1 \mathrm{~m} \times 1 \mathrm{~m}$ cross-section water tunnel which had a maximum speed of $1 \mathrm{~m} \mathrm{~s}^{-1}$. The cylinder diameter used was $1.78 \mathrm{~cm}$ and the flow velocity was varied between 0.04 and $0.60 \mathrm{~m} \mathrm{~s}^{-1}$, resulting in a Reynolds number range $\mathrm{Re} \approx 800-10,000$. A downstream plate, with a stream-wise length six times the diameter of the cylinder, was used to force the development of 2D vortex shedding. The hinged-rigid splitter plates were made using two plastic sheets of thickness $100 \mathrm{~mm}$ with a highly flexible plastic sheet of thickness $30 \mathrm{~mm}$ between them. A small part of the flexible sheet was left exposed between the rigid sheets on one side and the cylinder model on the other, and acted as the hinge. The effective stiffness of these composite plates was found to be sufficient to withstand the bending of the plates, and the observed motions were purely due to the bending at the hinge. The cylinder was mounted vertically so that gravity played no role in the motion. The splitter plate motions were visualized at rates up to $40 \mathrm{~Hz}$ using a CCD camera in conjunction with a halogen lamp or a PIV laser. Time traces of the displacement of the trailing edge of the splitter plate were obtained from image processing of the acquired images. The experiments showed that the splitter plate oscillations increased with Reynolds numbers at low values of Re, and were found to reach a saturation amplitude 
level at higher $\operatorname{Re}, \operatorname{Re}>4000$. The saturation tip amplitude level could be up to 0.45 times of the diameter of the cylinder.

\subsection{Indicators of harvested energy}

To enhance and optimize the energy output of a harvester, it is crucial to evaluate the harvested energy. If the harvesters are based on the same mechanism and in similar size, they can be evaluated by the magnitude of maximum or average power output. As there are different dimensions and mechanisms among various harvesters, it is unwise to assess the harvester simply based on those values. Instead, specific indicators shall be used. Two indicators are introduced herein to provide a unified evaluation of the performance of EH devices, regardless of the physical mechanisms of the transducer: energy output density and energy conversion efficiency.

The energy output density is defined as energy output per volume of a transducer, which indicates the efficiency in the use of the functional material. In a specific case, the output density can also be defined as the ratio of the energy output to the area/length of the transducer. The energy conversion efficiency is more complicated and several methods can be applied. The main idea is to calculate the ratio of energy output to the total available energy. Thus, the conversion efficiency refers to the degree that a harvester exploits the ambient flow. In [53], the mechanical energy that the flow transfers to the host structure is regarded as the total energy input $E_{0}$, which is calculated by 


$$
E_{0}=\int_{t} \sum_{i} F_{i} \dot{q}_{i} \mathrm{~d} t
$$

where $q_{i}$ and $F_{i}$ indicate, respectively, the generalized coordinate of the EH system and the corresponding generalized force. The total energy conversion efficiency $\mu$ is

$$
\mu=\frac{E}{E_{0}} .
$$

where $E$ is the harvested energy. The parameter $\mu$ can be used to design the configuration of the transducers to enhance EH performance. Based on [96], the total mechanical power available in uniform and steady fluid flow $P_{f}$ was proportional to the cube of the flow speed

$$
P_{f}=\frac{A_{f}}{2} \rho U^{3}
$$

where $A_{f}$ is the frontal area of the harvester in operation. Let $P_{0}$ and $P$ be the power that the structure received form the flow (corresponding to the energy in Eq. (25)) and the electric power output, respectively. The total efficiency is

$$
\mu=\frac{P}{P_{f}}=\frac{P}{P_{0}} \frac{P_{0}}{P_{f}}
$$

where the second ratio, on the right, is called "aeroelastic efficiency" and the first one is the "electromechanical efficiency" [96]. As expected, $\mu$ receives values $0 \leqslant \mu<1$.

A good practice, encouraged for future studies, is to use the energy output density and/or the conversion efficiency in the analysis of EH systems. In this work, due to the lack of information in the literature, it was found impossible to employ any of the two suggested indicators to classify the flow-induced vibration-based EH designs. Instead, 
the average power output is used as a complementary indication in this paper.

\section{Energy harvesting designs and implementations}

In this Section, the designs and implementations to generate electric energy from different flow-induced vibrations are reviewed. This Section is structured in a similar fashion to Section 2.

\subsection{Limit cycle oscillations of plates}

The idea to generate power from the flapping flags is shown in Fig. 32, where transducers are attached on the surface of the plates. Tang et al. [55] studied the energy transfer between the fluid flow and a plate in terms of various locations along the length of the plate and various vibration modes of the system, based on which they introduced the concept of "flutter mill" to represent EH from the plate, and compared it to a real horizontal axis wind turbine (HAWT). It was demonstrated that the flutter-mill can be designed to achieve higher performance with a more compact size. Dunnmon et al. [57] attached piezoelectric materials to the root of a cantilever flag to achieve $\mathrm{EH}$.

Simulations and experimental results of their study indicated that the system tested in Fig. 32 was able to obtain over $17 \%$ of the flow energy to which it was exposed. Doare et al. [97] studied the impact of the piezoelectric materials on the motion of the plate. It was observed that piezoelectric EH added rigidity and damping on the motion of the flexible plate. It was finally demonstrated that the modes of the plate, destabilized by the piezoelectric coupling, maximize the energy conversion efficiency. The response of 
the plate was also investigated by Akcabay et al. [52]. The effects of large beam deformations, membrane tension, coupled electromechanical responses, and base vibrations were considered. For a plate undergoing LCOs, high fluid loading, which is classically associated with destabilisation by damping, leads to a greater EH but with a weaker robustness to flow velocity fluctuations due to the sensitivity of the flapping mode selection [75]. Piñeirua et al. [98] and Xia et al. [26] investigated the influence of the arrangement of the piezoelectric electrodes along the plate surface and the inductive circuits on the dynamics of this fluid-solid-electric system and the EH efficiency, respectively. It was demonstrated that by optimizing the configuration of the electrodes along the length of the plate and carefully designing the circuits, the performance could be further enhanced. Apart from implementing piezoelectric effect and electromagnetic induction, Giacomello et al. [94] used IPMC to realize EH from the flapping flag. A schematic of the configuration of the flexible heavy flag and IPMC strip is shown in Fig. 33.

Li et al. [99] explored energy harvesting from a piezo-leaf consisting of a flexible beam subjected to cross flow. The piezo-leaf architecture amplified the vibration by an order of magnitude, making it appropriate for low-cost piezoelectric materials. Zhao et al. [92] investigated the LCOs of a wing-like structure subject to the cross flow and connected to a piezoelectric beam. Different aspect ratios of the structure were taken into account. Li et al. [29] compared the three configurations of the cantilevered plates 
shown in Fig. 34. The largest output power was still found in cross flow-induced configuration (Mode 1). This was caused by large bending and torsion. Hobeck et al. [100] further explored the interaction of two EH cantilevers to further amplify the power output. Specifically, two identical cantilever beams positioned side-by-side and oriented perpendicular to air flow were used as shown in Fig. 35. It was found that vibrations began when the two beams were placed in low velocity air flow at a particular orientation relative to each other and relative to the flow direction. Such configuration provided a robust type of flow-induced excitation over a large range of flow velocities.

\subsection{Galloping and vortex-induced vibrations of bluff bodies}

VIVs and galloping oscillations were investigated to generate energy due to their large in-plane amplitudes normal to the flow. The lock-in phenomenon, in which the vortex shedding frequency is entrained to the fundamental structural frequency, can be exploited to achieve resonance over a range of flow velocities. Such energy harvesters consist of bluff bodies with different cross sections, and transducers are coupled to the transverse DOF. EH could be achieved as the flow exceeds the threshold of the instability.

This EH design was introduced through the concept VIVACE (vortex-induced vibration aquatic clean energy), shown in Fig. 36, by Bernitsas and Raghavan [101]. It consists of a rigid circular cylinder mounted on linear springs. The harvester has been 
proved to be unobtrusive and robust, having high energy density, low maintenance, low life cycle cost and an expected 10 to 20 -year life.

One of the most important factors of VIVs and galloping oscillations is the crosssection geometry of the bluff body $[33,102]$. Several cross sections including square, pentagonal, cylindrical, triangular, and D-section exist, as summarized in Fig. 37. Kwon et al. [89] experimentally implemented a T-shape cantilevered structure to reduce the cut-in speed. An H-shape structure was numerically analyzed by Zhu et al. [59] for the same purpose. The effects of triangular and D-section were numerically studied in [64]. Compared to a cylindrical and pentagonal bluff body at $\mathrm{Re}=121-125$ [103], it was found that a D-shaped bluff body experienced a wider lock-in and a higher efficiency of about $15.9 \%$. The reason for this is that the D-shaped cross section resulted in an increase of the drag force with resonant vibration amplitude. In turn, this caused a stronger vorticity, a larger beam deflection, and ultimately a higher power output. Similar results were numerically and experimentally obtained for galloping vibrations using D-shaped, square and triangular cross sections at a higher Reynolds number of 10800 (at flow velocity $3.25 \mathrm{~m} \mathrm{~s}^{-1}$ ) in [104]. Based on the findings of an experimental and numerical activity carried out in [77], a recommendation was also given to use a square crosssection for wind galloping energy harvesters in laminar flow (at flow velocity $2.5 \mathrm{~m}$ $\mathrm{s}^{-1}$ ). The superiority of square cross section was attributed to the large lift curve slope. As opposed to a square cross section, in laminar flow the D-shape cross section had 
negative lift curve slope which is unable to generate the galloping phenomenon. The analysis carried by Meseguer et al. [105] showed that the more promising bodies were those with isosceles or approximate isosceles cross sections. Based on a rhombic body, Ibarra et al. [106] numerically explored the dependency of the instability on a geometrical parameter such as the relative thickness, and the reasons that this crosssection shape, that is generally unstable, shows a small range of relative thickness values where it is stable.

Sirohi et al. [80] numerically studied a triangular bluff body-based energy harvester with quasi-steady aerodynamics and implemented experiments to verify the results. It was concluded that a refined aerodynamic model was needed to include apparent mass effects for more accurate predictions. A similar analysis was done on a D-shape bluff body-based EH system [62]. It was observed that the geometry and the mass parameters, which determine the natural frequency of the system, play a significant role in the maximum power generated. Also, quasi-steady aerodynamics was sufficient to model the forcing during galloping. Abdelkefi et al. [31, 51, 70, 79, 82] studied the effects of the freestream velocity and electrical load resistance. It was found that the load resistance shifted the onset of synchronization to higher freestream velocities and the nonlinearities resulted in a hardening behaviour for some values of the load resistance. Bibo et al. [32] investigated the nonlinear force of the springs and showed advantages of soft and bi-stable restoring forces under specific cases compared to 
hardening ones.

Zhao et al. [107] compared the modelling methods including single DOF model, the Euler-Bernoulli distributed parameter model using single mode and the EulerBernoulli distributed parameter model using multi-modes and investigated the effects of wind exposure area of the bluff body, mass of the bluff body and length of the piezoelectric sheets. Comparison of the modelling methods was also conducted by Abdelkefi et al. [78]. Tang et al. [84] aimed at the modelling of the circuit and proposed an equivalent circuit representation approach capable of incorporating various interface circuits to predict the EH performance. Bibo et al. [108] established a universal relationship between a dimensionless version of the output power and the flow speed for galloping energy harvesters.

The effect of non-rigid bonding of the piezoelectric transducer to the host structure on strain transfer was investigated by Akaydin et al. [96]. The schematic of the EH system and the electrode configuration are shown in Fig. 38. While the rigid bonding assumption, whereby the adhesive layer has zero thickness and infinite shear strength, caused a significant overestimation of the measured power, a non-rigid bonding model gave a better agreement. Abdelkefi et al. [109] studied the influence of ambient temperature on the onset speed of galloping and the level of the harvested power. The temperature was found to influence the properties of piezoelectric materials including the piezoelectric and dielectric constants and the Young's modulus. It was shown that at 
lower wind speeds, more energy was harvested at higher temperature values, while at relatively higher wind speeds, more energy was generated at lower temperatures. Yan et al. [110] took base vibration into account and studied its effects. The numerical results showed that the existence of the galloping phenomenon decreased the tip displacement and power output at the resonance region while increasing the displacement and output off-resonance region. The former was unfavourable for decreasing the maximum power output, while the latter was beneficial for enlarging the applicable flow velocity range of the harvester.

Zhao et al. [111] proposed a two DOF piezo-aero-elastic energy harvester with a cut-out cantilever and two magnets to reduce the cut-in wind speed and enhance power output, as shown in Fig. 39 (a). Ewere et al. [90] introduced an impact stop to reduce bending stresses and improve the fatigue life of traditional EH systems based on galloping oscillations. Vicente-Ludlam et al. [112] introduced a dual mass to a galloping-based EH system (Fig. 39 (b)). The results showed an improvement in the efficiency of energy extraction and a broadening of the values of the incident flow velocities with respect to that of the single mass configuration.

Rostami et al. [113] modified the concept of the VAACT introduced in [30] with a flapped profile shape. The profile is a flat plate with two end flaps, which are tilted in the opposite direction. This shape not only causes an increase of the lift and drag forces, but also changes the centre of pressure which moves outward. Consequently, more 
yawing moment is generated. The effects of the harvester moment of inertia and the flap were experimentally studied to improve the autorotation characteristics. It was found that the VAACT has an efficiency of $33 \%$ in very low head current, and that the flap increases the power coefficient significantly, by a factor of up to 5, compared to flat plate type of [30].

\subsection{Limit cycle oscillations of wing sections}

EH from aeroelastic LCOs of a wing typical section is illustrated in Fig. 40. The aerofoil with two DOFs in pitch and plunge is supported by elastic springs with nonlinear stiffnesses. The transducers, either piezoelectric or electromagnetic, are attached to the plunge DOF. Depending on whether there are motors to drive the wing section, the aerofoil-based EH system may be classified into fully prescribed, semipassive and fully passive [6]. The focus in this work is on the passive designs where the oscillations of the wing section are determined by the fluid-structure interaction. In addition, those $\mathrm{EH}$ designs were developed exploiting either the flapping motion of the aerofoil based on various mechanisms [114] or the deformation of the supporting devices due to the aerofoils vibrations [115].

Bryant et al. $[34,116]$ implemented an aerofoil-based EH which consists of a wing section supported by a piezoelectric beam and a pin joint via ball bearings. The mechanism ensures that the aerofoil undergoes pitch and plunge motions, as shown in

Fig. 41. The comparison between aerofoil-based and plate-based EH systems from wind 
tunnel tests was assessed in [116]. The equations of motion of the EH system were used to calculate the linear flutter speed and the effects of flow velocity on flutter frequency and power output [58]. Methods for expanding the operating envelope of the power harvester and reducing the linear flutter speed were proposed [34, 117, 118].

Nonlinearities are significant factors for the design of an aerofoil-based EH system. Erturk et al. [85, 119] stressed the importance of the nonlinearities of such EH system based on a linear model. Hardening cubic stiffness is usually considered or assumed in the aerofoil-based energy harvesters [54, 56, 63, 120-122], the influence of which was studied in $[56,121]$. The effects of free-play on the pitch DOF were investigated by De Marqui Jr et al. [123] and Abdelkefi et al. [93]. Particularly, Sousa et al. [124] and Anicézio et al. [125] studied the EH system with the combination of the two nonlinearities via piezoelectric and magnetic transduction, respectively. This combination of two nonlinearities was also studied by Bae et al. [88] and Wu et al. [115]. It was demonstrated that the free-play nonlinearity reduced the cut-in speed while the hardening stiffness helped in obtaining persistent oscillations of acceptable amplitude over a wider range of airflow speeds. The aerodynamic nonlinearities were also considered in the study of aerofoil-based energy harvesters [74]. Apart from the structural and aerodynamic nonlinearities, the nonlinearity of transducers was studied in [72]. Results showed that although the limited stroke of the magnets and the undesired mechanical friction reduced the harvested energy, the nonlinearities of the magnetic 
forces and fluxes could represent an effective advantage, in particular, in a multi DOF system subject to a large frequency bandwidth input or random excitations.

The aerofoil-based energy harvesters can be arranged into arrays to further improve the power output. The configuration with two harvesters was proposed by Mahtani et al. [126] and Bryant et al. [91]. It was proved that the power output of a second, trailing harvester can be increased by up to $27 \%$ of its original output due to the presence of a first, leading harvester oscillating at a particular location upstream.

The base of the EH system influences the performance. Bryant et al. [127] studied the effects of the base flexibility on the linear flutter speed, frequency and power output. It was found that the harvester mounted on a compliant host structure had a cut-in speed that was approximately $12 \%$ less than that for the stiff structure, and a cut-in frequency that was about $5 \%$ lower. Also, the stiff structure case showed $24 \%$ more power output than that of the compliant case. Bibo et al. considered the excitation of the base and corresponding effects on the performance [128]. Below the flutter speed, the response of the harvester was periodic and the flow contributed to amplifying the influence of the base excitation. Beyond the flutter speed, as the base excitation was small and/or as the excitation frequency was not close to the flutter frequency, the response was quasiperiodic and the output power was hence reduced. As the excitation amplitude is large enough, the response became periodic and the output power increased exhibiting little dependence on the amplitude of the base excitation. 
Most of the designs were based on the piezoelectric effect. The use of electromagnetic induction followed [71, 72]. De Marqui Jr et al. compared aerofoilbased EH using those two transduction mechanisms [129]. The piezoelectric effect

offers a convenient way to harvest energy from structural deformations. The mechanism depends solely on the magnitude of deformation and is independent from the vibration frequency. On the other hand, the electromagnetic induction is strongly related to the kinetic energy from relative motions, and is strongly dependent upon the excitation frequency. An EH design with both approaches was proposed [86]. Specifically, the piezoelectric patches were attached at the root of the cantilevered beam while the coilmagnet arrangements were placed at the tip of the beam.

To further improve the energy output, some studies looked into modifying the EH device. Dias et al. [87] studied EH of an aerofoil with a free control surface (Fig. 42 (a)). Zhao et al. [130] enhanced the power output via using a beam stiffener (Fig. 42 (b)). The principle was to amplify the electromechanical coupling by magnifying the slope of the mode shape of the harvester at the edge of the transducer, which was achieved by stiffening the substrate to the right of the transducer.

\subsection{Vortex-induced vibrations generated in wake vortices}

EH from VIVs can be traced back to the concept of the EH eel [131], which consists of a piezoelectric membrane behind a bluff body, Fig. 43 . The following designs are concerned with connecting the beam or plate structures to the bluff body to generate 
vibrations due to the von Kármán's vortex street [95, 132-134]. Sivadas et al. [135] investigated different characteristic dimensions and shapes for the bluff body to seek a higher synchronized region of frequencies for the oscillation amplitudes. Numerical results showed a wider lock-in bandwidth and higher average power for the cylindrical bluff body compared to the triangular and pentagonal ones.

Akaydin et al. [40] placed a piezoelectric beam as the downstream structure to harvest energy from VIVs. The periodic force generated by the vortices shed off the cylinder was tuned at the resonance of the vibration modes of the piezoelectric beam. Therefore, the energy harvester underwent large amplitude forced vibrations and generated periodic energy output. Goushcha et al. [136] experimentally studied the interaction of individual and multiple vortices with a similar structure except that an audio speaker was used to generated controlled vortices.

The idea to exploit wake galloping phenomena was proposed by Jung et al. [41]. The wake galloping phenomenon has advantages for occurring at relatively low wind speeds and having a wide range of wind speeds with large but finite amplitudes, as already observed in the typical velocity-amplitude curve of different aerodynamic instability phenomena [41] (Fig. 15). The schematic of Fig. 44 shows the installation of transducers along the transverse DOF of the second cylinder to harvest energy from wake galloping. The applicability was demonstrated by Jung et al. [41]. Abdelkefi et al. $[137,138]$ used a square section downstream bluff body to study EH from wake effects. 
It was shown that the range of speeds over which energy was harvested could be significantly widened. The level of enhancement was dependent on the wake effects as determined by the size of the upstream cylinder and the spacing distance between the two cylinders. Depending on the placement of the harvester, an enhanced piezo-aeroelastic system could be further designed with an appropriate selection of the diameter of the cylinder and the electrical load resistance.

Table 2 compares several EH designs and implementations introduced in Section 4.1 to 4.4, in which several aspects of the EH systems are compared, including the sizes of the harvesters, power outputs and corresponding flow velocity. It can be seen that the design of all energy harvesting systems vary in dimensions from several millimetres to hundreds of centimetres. Generally, the power outputs, which increase with the flow velocity and dimensions, are in the order of mill-watt or microwatt. Harvesters may therefore find potential applications in micro-electronics. In most cases, the harvesters based on the galloping or flutter phenomena work at a wider flow velocity range and generate higher power outputs compared with VIV-based designs. However, the design of VIV-based harvesters is more flexible in its applications than that based on the mechanism of flutter or galloping. Moreover, the EH systems with PZTs or PDVFs generate more power compared to those based on electromagnetic induction-based harvesters. This may be the reason that the piezoelectric effect is more widely used for flow-induced vibration-based EH than the electromagnetic induction. 


\subsection{Turbulence and other variable flow-based vibrations}

Harvesting energy from atmospheric turbulence has the advantage that turbulence is a natural phenomenon commonly encountered in flight. Unlike other mechanisms overviewed in the previous paragraphs, turbulent-induced vibration-based harvesters do not require any upstream bluff body to generate wake vortices. The authors' opinion is that atmospheric turbulence offers a virtually unlimited source for structural vibrations, similarly to the availability of solar power.

Litak et al. [139] numerically studied EH from a turbulent wind source. The turbulent airflow was considered as a bounded Gaussian white noise. It was found that increasing the noise level led to the stochastic resonance, i.e. frequent hopping between the potential barrier (corresponding to the stable states of the system). It was observed that the response amplitude and power output were enhanced. Goushcha et al. [140] investigated $\mathrm{EH}$ of a piezoelectric beam from turbulence. The orientation of the beam relative to the incoming flow and the wall was found to be a critical parameter affecting the energy output. Abdelkefi et al. [138] used an upstream mesh to generate turbulence exciting a uni-morph piezoelectric cantilever beam with a square cross-section tip mass.

Sohn et al. [141] and Wang et al. [142, 143] studied EH of piezofilms under a fluctuating pressure source. In Fig. 45 a pressure chamber is shown with a flexible diaphragm connected to a flow source by a glass tube. By connecting the energy harvester to an ambient flow source, which was capable of providing the pressure 
variation in the pressure chamber, the oscillating movement of the diaphragm in upward direction with an attached transducer made EH possible. Vatansever et al. [144] focused on the various wind speeds and water droplets. It was proved that piezoelectric polymer materials could generate higher voltage/power than ceramic based piezoelectric materials when aiming at variable flow conditions. Fei et al. [145] implemented a flexible belt instead of membranes to harvest energy from random flow. An EH device inspired by music-playing harmonicas is shown in Fig. 46, where a piezoelectric beam is activated by the blown airflow within a cavity [146, 147]. The intermittent airflow creates a periodic pressure variation in the chamber which in turn generates LCOs of the cantilevered beam.

\subsection{In-aircraft energy harvesting implementations}

As to aircraft, aeroelastic vibrations are commonly generated during flight. Apart from flutter and LCOs discussed in Section 4.3, aeroelastic responses to various dynamic loadings are generally experienced and may be potential energy sources during flight. Primary attention is devoted to external aerodynamic loadings such as atmospheric turbulence or gusts. These loadings are essentially random in nature and must be treated accordingly. Erturk et al. [148] exploited EH of a uni-morph cantilever with macro-fibre composites and presented a distributed-parameter electromechanical model. Wind tunnel experiments were also performed for EH from a flow-excited morphing aerofoil. De Marqui Jr et al. [149] built an EH model for a wing-like plate 
with embedded piezoceramics to study the unsteady aeroelastic responses and electric energy output. While harvesting energy from wing flutter via piezoelectric transducers, it was observed that the time history of the tip displacement of the wing showed a decaying behaviour. This was attributed to the shunt damping effect, i.e. the structural vibration was damped through dissipating Joule heat over the resistances in the EH circuit. Then, the authors investigated the use of segmented electrodes to avoid cancellation of the electrical outputs of the torsional modes $[150,151]$. An optimization problem was solved in [76] where the aluminium wing spar of a UAV, shown in Fig. 47, was modified to obtain a generator spar by embedding piezoceramics for the maximum electrical power without exceeding a prescribed mass addition limit. The frequency domain model was developed by De Marqui Jr et al. [152]. Vieira et al. [153] performed nonlinear modelling and analysis of an electromechanically coupled cantilevered helicopter blade. Abdelkefil et al. [154] generated a model to study EH from wing morphing. It was demonstrated that there was enough power to operate a camera. Xiang et al. $[53,155]$ built a model which considered a wing spar with piezoelectric patches and discrete gust loads, as shown in Fig. 48. Based on the model, the effects of the geometric parameters of the transducers and the wing were studied. Results showed that high energy could be harvested as the piezoelectric materials were placed at the wing root. For a specific gust disturbance, an optimal thickness and length of the transducers was found. 
Pozzi et al. [156] studied EH from gust loads by implementing piezoelectric patches onto the skin instead of the wing spar. A strain map was elaborated, which details the distribution of strain on the wing skin in response to gust loading, indicating the optimal locations for the harvesters. The effects of harvester thickness, size and adhesive thickness were investigated. Experiments were also performed in a tensile testing machine [157]. It was found that macro-fibre composites had a lower power density and were more resilient than monolithic transducers.

Magoteaux et al. [158] used the entropy generation analysis to study a small UAV with and without EH from solar and vibrational energy. This method was used to evaluate the amount of dissipation in the heat and mass transfer processes within the thermodynamic cycle. The UAV subsystems including the battery, motor, voltage regulator, speed controller, receiver and servo systems were considered. The entropy of these subsystems was related to the heat generated by the subsystem, ambient sink temperature, the masses of the subsystem components, the flight condition, aerodynamic lift, aspect ratio of the wing and the efficiency of the motor and propeller. The analysis showed that with a predefined reconnaissance mission (including a dash to the target, loitering, perching and recharging, and a dash back to the starting location), the UAV with EH generated less entropy, i.e. the UAV consumed less energy, whereas the UAV without EH outperformed the former in total flight time at the target. It was demonstrated by several tests that in the future the addition of the solar and piezoelectric 
devices would be advantageous for a small UAV. Anton et al. [159] investigated the possibility of harvesting vibration and solar energy in an UAV. Experimentation was carried out on a remote controlled glider aircraft with a $1.8 \mathrm{~m}$ wing span. The aircraft was modified to include two piezoelectric patches placed at the roots of the wings and a cantilevered piezoelectric beam installed in the fuselage to harvest energy from wing vibrations and rigid body motions of the aircraft, as well as two thin film photovoltaic panels attached at the top of the wings to harvest energy from sunlight, as shown in Fig. 49. Then, the concept of multifunctional wing spar shown in Fig. 50 was introduced $[160,161]$. The proposed self-charging structures contained piezoelectric layers for power generation, thin-film battery layers for energy storage, and a central metallic substrate layer, arranged in a bimorph configuration. The modelling and analysis was performed together with experiments $[162,163]$.

Implementing EH into an UAV leads to additional mass and increased drag which may adversely affect the flight performance of the aircraft. To study the influence of EH, Anton et al. [164] applied a formulation to describe the changes to the flight endurance of a UAV based on the power available from added harvesters and the mass of the harvesters. Theoretical and experimental results showed that using thin-film solar panels in a multifunctional manner, where some structure mass is removed to compensate for the addition of the harvesting system, could lead to an increase in flight endurance. As the mass of both solar panels and piezoelectric transducers was removed 
in the structural mass, a loss in flight endurance occurred unless a fully multifunctional design was performed. These results demonstrated the necessity of fully multifunctional designs for the integration of piezoelectric vibration harvesting into UAVs.

Other loadings of interest in flight for EH may be impulsive or discrete loadings such as the sudden loading due to manoeuvring of a flight vehicle as a result of control surface rotation, or the impact onto the landing gears. Erturk et al. [165] studied EH from the vibrations of an L-shape beam aiming at the application to landing gear. Note that $\mathrm{EH}$ discussed here is beyond the scope of that exploiting flow-induced vibrations, but having potential for EH on UAVs.

\section{Applications of energy harvesting}

\subsection{Power supply for wireless sensor networks}

In the last few decades, there has been an increasing interest on micro wireless electronic devices. Wireless sensor networks (WSNs) are formed from a collection of sensing nodes which communicate with one another, typically through wireless channels, in order to collect spatially distributed data about the environment. Such networks have the potential to provide better quality data than single or small numbers of individual sensors in applications such as natural monitoring, process monitoring, security and surveillance [166]. There are widespread needs for the use of WSNs for structural health monitoring, especially in areas like oil and gas pipeline monitoring, bridge maintenance companies, as well as railway, automotive, aircraft, marine and 
military industry [4]. An example of photograph and schematic of wireless sensor module is shown in Fig. 51 [167].

In order to be cost effective in many applications, WSNs must be low cost and low maintenance. This presents challenges in terms of sensor calibration, packaging for survival in harsh environments and, particularly, the efficient supply and use of power. WSNs research has predominantly assumed the use of a portable and limited energy source, i.e. batteries, to power sensors. Without energy, a sensor has essentially no use and cannot contribute to the utility of the network as a whole. Consequently, substantial research efforts have been spent on designing energy-efficient networking protocols to maximize the lifetime of WSNs. However, there are emerging WSN applications where sensors are required to operate for much longer durations like years or even decades after they are deployed. Examples include in-situ environmental/habitat monitoring and structural health monitoring of critical infrastructures and buildings, where batteries are difficult or impossible to replace/recharge [168].

Up-to-date technologies allow WSNs to reduce power requirements to the range of tens to hundreds of microwatts [170]. Such low power consumption leads to the possibility of powering WSNs by converting ambient energy from the environment and hence eliminates the need for batteries and extend the lifetime indefinitely $[116,145$, $168-169,171,172]$.

A generic WSN node with EH technology is illustrated in Fig. 52 [166]. The power 
requirement of each element depends on the particular application. So it is difficult to generalize about which parts of the node consume the most. For instance, it is commonly the case that actuators, if present, consume a large proportion of the total power. In addition, consumption depends on the operating mode of a device such as whether it uses sleep mode to reduce consumption and how frequently it transmits and receives data [166].

A strain EH system for powering an embedded wireless sensor network without batteries was demonstrated in [173]. A composite material specimen laminated with unidirectional aligned piezoelectric fibres is shown in Fig. 53. The specimen was loaded in three-point cyclic bending using an electrodynamic actuator operating at different frequencies. Strain energy was stored by rectifying piezoelectric fibre output into a capacitor bank. When the capacitor voltage reached a pre-set threshold, charge was transferred to an integrated, embeddable WSN. The times required to accumulate sufficient charge to accomplish data transmission was evaluated. For peak strains of $150 \mu \varepsilon$, the time to transmit was 30 to 160 seconds (for 180 to $60 \mathrm{~Hz}$ tests).

WSNs need to be designed to be compatible with EH and achieve better performance. A distributed framework was presented in [174] for the sensor network to adaptively learn its energy environment and give localized algorithms to use this information for task sharing among nodes. The framework allowed the system to exploit its energy resources more efficiently, thus increasing its lifetime. These gains were in 
addition to those from utilizing sleep modes and residual energy based scheduling mechanisms. Similarly, reference [175] focused on making WSNs adaptive to the changing environment. Results showed that better performance could be achieved on a broader class of energy source data sets by implementing adaptive control theory. A low-power maximum power point tracker (MPPT) circuit specifically designed for WSNs was proposed in [176] which was specifically a power transferring circuit for optimally conveying solar energy into rechargeable batteries even in not optimal weather conditions. MPPT was also used in the circuit of [177] together with a super capacitor based energy storage system for WSNs. This design was modular and enabled composition of multiple energy harvesting sources including solar, wind, thermal, and vibration, each with a different optimal size. Reference [178] presented an optimal energy management policy for the solar-powered sensor node that used a sleep and wakeup strategy for energy conservation. The problem of determining the sleep and wakeup probabilities was formulated as a bargaining game. The Nash equilibrium was used as the solution of this game.

Nowadays many energy harvesters that are small enough for use in wireless sensors are most likely able to provide only enough energy to power sensors sporadically and not continuously [168]. Thus, the challenge of sensor nodes is the need to exploit the sporadic availability of energy to quickly sense and transmit the data. Also, another challenge is the optimization for power consumption minimization [179] and power 
output maximization which ensures the system's energy autonomous capability by implementing EH technology. Flow-induced vibration-based EH systems have the potential to tackle the aforementioned challenges.

\subsection{Simultaneous energy harvest and control}

Some energy harvesters themselves, e.g. piezoelectric ceramics due to the piezoelectric effects, can be implemented onto the structure and serve as both energy harvester and actuator. Therefore, attention has been given to the control aspects via EH system. Di Monaco et al. [180] implemented semi-active control to optimize the power harvested by an electro-mechanical energy harvester. The results showed that a timevarying damper was more effective than a passive one and an on-off semi-active model in absorbing power at any non-resonant frequency. It was also demonstrated that the optimized periodic time-varying damper could harvest more power at off-resonance compared to an arbitrary periodic time-varying damper, which was chosen to have the same transmissibility at resonance. Afsharfard et al. [181] used piezoelectric elements as single unit impact dampers, which was capable of vibration control while EH. An analytical approach was presented to formulate the voltage generation and the vibrations suppression.

Furthermore, the harvested energy can be stored and used to provide necessary power for discrete control to the host structure. As the power generated by EH approaches is relatively low, the control methods should be properly selected. Wang et 
al. [182] presented a summary of passive, semi-passive, semi-active, and active control methods for schemes using harvested energy as the main source of energy to suppress vibrations via piezoelectric materials. The key point of such research lies in developing minimum energy control laws while maximizing the amount of energy harvested.

Based on the concept of multifunctional composite wing spar, Wang et al. [183] proposed simultaneous $\mathrm{EH}$ and vibration control. The intention was to design a composite wing spar for a small UAV which was able to harvest energy itself from ambient vibrations during normal flight along with available sunlight. If the wing experienced any strong wind gust, it would sense the increased vibration levels and provide vibration control to maintain its stability. The proposed multifunctional composite wing spar integrates a flexible solar cell array, piezoelectric wafers, a thin film battery, and an electronic module into a composite sandwich structure, as shown in Fig. 54. The piezoelectric wafers act as sensors, actuators, and harvesters. Special attention was given to the self-contained gust alleviation with the goal of using available energy harvested from ambient vibrations. A reduced energy control law was implemented to reduce the actuation energy and the dissipated heat. This law integrated saturation control with a positive strain feedback controller and was represented by a positive feedback operation amplifier and a voltage buffer operation amplifier for each mode. Theoretical analysis and numerical modelling also showed that the tip displacement due to a wind gust disturbance could be reduced by 28 and $37 \mathrm{~dB}$ for the 
first and the second modes, respectively, using energy harvested from ambient wing vibrations. In order to control a 1 second time span wind gust, 40 seconds of harvesting were needed.

The multifunctional wing spar is a promising concept on wing-based EH and its corresponding application, but it is still not capable of EH from normal atmospheric disturbance effectively. The dimensionless tip displacement of the multifunctional composite wing spar with frequency and harvested power spectrum at one optimal load resistance are shown in Fig. 55. As it can be seen, within the frequency range from $5 \mathrm{~Hz}$ to $400 \mathrm{~Hz}$, two maximum power outputs close to $1 \mathrm{~mW}$ can be obtained near the two resonance regions. When the excitation frequency slightly deviates from the resonance frequencies, the power output will greatly decrease (down to $10^{-5} \mathrm{~mW}$ according to Fig. 55 (b)). By comparing the PSD spectrum of atmospheric turbulence shown in Fig. 16 (a), it is obvious that it is difficult for such EH system to fully exploit the atmospheric disturbance in flight.

Other than wing-based EH, Bryant et al. [184] attached aerofoil-based piezoelectric $\mathrm{EH}$ as devices onto the control surface of a wing, aiming at creating self-powered actuation of aero-structure surfaces such as tabs, flaps, spoilers, or morphing devices. Two configurations for such a system had been investigated experimentally. The first, shown in Fig. 56, demonstrates that the EH device and the aero-structure control surface to be actuated were configured as separate and independent subsystems. The second 
concept shown in Fig. 57 integrates the two functions into a single device to simultaneously use the oscillating piezoelectric structure itself as both an energy harvester and a tab-like control surface. Actuation tests on the first configuration showed that the system could produce more than 55 degrees of tab deflection over approximately 0.7 second after the energy harvester had charged the storage capacitor for 235 seconds in a $32.2 \mathrm{~m} \mathrm{~s}^{-1}$ flow. As the EH device experienced a nonzero net angle of attack to the flow, it showed that the harvester continued to operate even when its host control surface was rotated to very large angles of attack over 50 degrees, though its power output was reduced by $56 \%$ compared to the zero angle of attack case. Upon the second configuration, the deflected control surface with the oscillating harvester produced similar variations in lift and pitch moment with deflection angle when compared to a static device. The drag generated by the oscillating energy harvester was shown to depend highly on the LCO amplitude excited by the flow. Large amplitude LCO in the energy harvester generated maximum power, but resulted in significant increases in drag on the wing section. At lower wind speeds the wind tunnel tests demonstrated that up to $4.5 \mathrm{~mW}$ of power could be extracted from the flow by smaller oscillation amplitudes that produced no measurable increase in drag on the wing section. This highly efficient energy harvesting regime persisted even when the energy harvester was rotated with the host control surface to large angles of attack up to 50 degrees. 
By attaching the $\mathrm{EH}$ harvesters onto the wing, $\mathrm{EH}$ can be achieved in flight even when no atmospheric disturbance exists. The demonstration of tab actuation powered by EH from ambient flow makes this design a potential application of wing-based EH. However, the design does not fully meet stringent requirements for aerospace applications. The additional EH devices are likely to affect the whole aerodynamic configuration of the wing if they are exposed directly to the airflow, not to mention the effects on the wing such as the additional mass, the changes in the centre of gravity and aerodynamic centre, and the structural complexity. Furthermore, the installation of EH devices results in the coupling between the host structure and harvesters, which may need revision or redesign on both the harvesters and host structure. Also, the EH device works as LCOs are generated, which is commonly a situation avoided in any aircraft operation. Although the increase of drag is small as the operating amplitude is relatively low, it is likely to bring negative effects on the performance of the aircraft. This can be unacceptable especially for new generation UAVs that pursue great performance during flight.

\section{Summary and Challenges}

A review of EH designs and implementations based on several flow-induced vibration phenomena has been performed. A number of applications of the EH systems was discussed, with a particular emphasis on $\mathrm{EH}$ from aeroelastic vibrations and the exploration of using on-board harvested energy to provide control or actuation. Of all 
the references reviewed in this journal, only the works presented in Refs. [53, 76, 148, $151-153,155,156,159,162-165,183,184]$ addressed flow-induced vibration-based EH for aircraft or UAV/MAV applications. In these references, concepts and experimental prototypes have been introduced to harvest energy from base excitation, gust and other sources of vibrational energy by equipping the wing with a number of transducers. The discussion in Section 5.2 has shown promising results from $\mathrm{EH}$ simulation and experiments, but it is recognized to date that this technology has not achieved full testing, demonstration, and verification on realistic aerospace applications. Several challenges that hinder the future development and application of flow-induced vibration-based $\mathrm{EH}$ in the aerospace are discussed in the following sections.

\subsection{Modelling of nonlinear coupled aero-electro-elastic model}

The design and analysis of EH systems rely on the coupled model that describes the interactions between structural dynamics, transducers, aerodynamics and atmospheric disturbance. A large part of the work in the literature is limited to the electromechanical coupling and EH from base excitations. However, a fully coupled model is needed to accurately predict the nonlinear couplings, and the influence of nonlinearities on the overall system performance. The widespread use of linear (or linearized) models is due to the fast turnaround time of the calculations and the availability of semi-empirical corrections to account for nonlinear effects. To design, analyse, and optimize the performance of the $\mathrm{EH}$, accurate numerical models that include nonlinearities and 
couplings between disciplines should be employed. In subsonic flow, the aerodynamic nonlinearities are from flow separation and vortex shedding, and dynamic stall needs to be considered for the harvesters undergoing large amplitude flow-induced vibrations. The structural nonlinearities may result from geometrically-large deformations, freeplay, hardening and softening springs. Beam and plates are common structural models for wing structures, but have a limited prediction capability if deformations of the wing skin are significant, as in the case of membrane wings. The nonlinearities of the transducers may also need to be considered during the design process, enhancing the physical insights on the coupled mechanisms driving the generation of energy. Physicsbased coupled models introduce numerical difficulties to seek for a solution and come at the cost of high computing times. Acceleration techniques are highly needed to fully exploit the physical insights provided by these simulation tools in the design process to optimize the harvester design for better performance than available today.

\subsection{Experimental testing}

Flow-induced vibration-based EH is a cross-disciplinary problem involving several disciplinary fields: structures, fluids, electromechanical phenomena, and power management, among others. As discussed in Section 5.2, the use of EH may influence the normal operating capability of the host structure, and introduce a strong coupling between the host structure and the EH device. Despite practical challenges, experimental testing provides valuable insights that may be used as a design opportunity 
to corroborate numerical activities. It is recommended that experiments should be implemented in parallel with simulations during the design process.

\subsection{Enhancing harvested energy and performance}

There is a requirement to increase the energy outputs of EH systems. The harvested vibrational energy is low compared to the energy needed to operate sensors and electronics installed on-board aerial platforms. In addition, various aspects of the performance of the harvester must be considered in the design phase according to the operating environment and mission requirements, including reducing the cut-in speed, extending the operating bandwidth, enhancing output density/conversion efficiency, improving robustness and increasing the life time. It is worth noting that a trade-off must be made between those indicators, generally. To achieve higher energy output and better performance, the systematic and parametric analysis of the EH system is needed to obtain optimal designs.

\subsection{Scaling effects on the energy harvesting performance}

The flow-induced vibration-based EH designs have been demonstrated practically on small scale prototypes, which makes them promising to be embedded in small scale systems to supply power for micro devices or MAVs. However, as the harvesters are scaled up to be used for larger host structures, e.g. an unmanned aerial platform of few meters wing span for long mission profiles, the scaling effects on both dynamic responses and $\mathrm{EH}$ performance have to be analysed fully. A second consideration of a 
scaled-up harvester is the added weight and complexity resulting from its installation on the airframe structure compared to the amount of harvested energy.

\subsection{Design and power management of host systems}

Apart from the development aiming at the energy harvesters themselves, the host systems may also need optimization on power management or energy saving, in order to fit EH application. WSNs give an example to be compatible with EH methods when strategies are used to make every single node capable of reducing power consumption and adjusting working cycle according to the amount of energy harvested. Power arrangement can also be implemented onto mission payload on aircraft including small electronic devices. However, as to an aircraft, it is unrealistic to change the design of a structural component to accommodate a EH device. Thus, it may be important to take EH design into consideration at the design phase of the host systems.

\subsection{Balance between advantages and disadvantages of energy harvesting}

Several EH solutions reviewed in this work, despite the claims of the corresponding references, are found inadequate for use in any aerospace application. General requirements, which shall be considered as design constraints, are: a) the added weight penalty of the EH device shall be minimum, and the amount of energy harvested per unit weight shall be maximum; b) the integration of the EH device on the airframe shall be seamless, requiring no extra complexity or design changes to the baseline airframe;

c) the installation of the EH device shall not degrade the overall aerodynamic 
performance of the airframe. External applications of the EH that interfere with the flow around the airframe are questionable, as drag will ultimately increase; and d) the EH device shall operate during the entire mission profile of the airframe, and shall be optimized accordingly.

The design of EH technologies for aerospace applications is driven by multiple constraints. It is suggested to utilize a comprehensive model that includes the $\mathrm{EH}$ system itself together with other constraints such as the weight and a performance target. Also, as flow-induced vibrations are the main energy source considered, harvesting more energy requires larger vibrational amplitudes, in turn, causing potential fatigue damage to the host structure.

In conclusion, further improvements in flow-induced vibration-based EH depends on the ability to: a) employ better designs that exploit nonlinear couplings as a design opportunity rather than an operating limitation; b) conduct concurrent theoretical and experimental analysis to leverage on the physical insights obtained by the various investigations; and c) evaluate and assess various harvester concepts based on their added weight penalty and complexity of integration. Finally, it is recognized that the development of functional materials, high-performance batteries, and high-efficiency structural configurations are likely to benefit the introduction of flow-induced vibrationbased EH for unmanned aerial operations. 


\section{Acknowledgement}

The financial support from the Royal Academy of Engineering for the project "Fast Nonlinear Aeroelastic Search for Loads Assessment" (NCRP/1415/51) is gratefully acknowledged. The authors also gratefully acknowledge the support from the National Natural Science Foundation of China under grant Nos. 11402014 and 11572023.

\section{References}

[1] Pines DJ, Bohorquez F. Challenges facing future micro-air-vehicle development. J Aircraft 2006; 2 (43): 290-305.

[2] Bafleur M, Dilhac J. Towards energy autonomy of wireless sensors in aeronautics applications: smarter collaborative project. In: IEEE International Conference on Green Computing and Communications and IEEE Internet of Things and IEEE Cyber, Physical and Social Computing, Beijing, China, 2013.

[3] Thomas JP, Qidwai MA, Kellogg JC. Energy scavenging for small-scale unmanned systems. J Power Sources 2006; 2 (159): 1494-1509.

[4] Marsic V, Zhu M, Williams S. Wireless sensor communication system with low power consumption for integration with energy harvesting technology. Telfor Journal 2012; 2 (4): 89-94.

[5] Raghunathan V, Kansal A, Hsu J, Friedman J, Srivastava M. Design considerations for solar energy harvesting wireless embedded systems. In: 4th international symposium on Information processing in sensor networks, Los Angeles, California, 
2005.

[6] Young J, Lai JCS, Platzer MF. A review of progress and challenges in flapping foil power generation. Prog Aerosp Sci 2014; (67): 2-28.

[7] Xiang J, Yan Y, Li D. Recent advance in nonlinear aeroelastic analysis and control of the aircraft. Chinese J Aeronaut 2014; 1 (27): 12-22.

[8] Li D, Guo S, Xiang J. Study of the conditions that cause chaotic motion in a twodimensional airfoil with structural nonlinearities in subsonic flow. J Fluid Struct 2012; (33): 109-126.

[9] Li D, Xiang J. Chaotic motions of an airfoil with cubic nonlinearity in subsonic flow. J Aircraft 2008; 4 (45): 1457-1460.

[10] Li D, Guo S, Xiang J. Modeling and nonlinear aeroelastic analysis of a wing with morphing trailing edge. Proceedings of the Institution of Mechanical Engineers, Part G: Journal of Aerospace Engineering 2013; 4 (227): 619-631.

[11] Badcock KJ, Timme S, Marques S, Khodaparast H, Prandina M, Mottershead JE, Swift A, Da Ronch A, Woodgate MA. Transonic aeroelastic simulation for instability searches and uncertainty analysis. Prog Aerosp Sci 2011; 5 (47): 392423.

[12] Fichera S, Jiffri S, Wei X, Da Ronch A, Tantaroudas N, Mottershead JE. Experimental and numerical study of nonlinear dynamic behaviour of an airfoil. In: ISMA International Conference on Noise and Vibration Engineering, Leuven, 
Belgium, 2014.

[13] Li D, Xiang J, Guo S. Adaptive control of a nonlinear aeroelastic system. Aerosp Sci Technol 2011; 5 (15): 343-352.

[14] Li D, Guo S, Xiang J. Aeroelastic dynamic response and control of an airfoil section with control surface nonlinearities. J Sound Vib 2010; 22 (329): 47564771.

[15] Wang Y, Li F, Da Ronch A. Adaptive feedforward control design for gust loads alleviation of highly flexible aircraft. In: AIAA Atmospheric Flight Mechanics Conference, Dallas, Texas, 2015.

[16] Wang Y, Da Ronch A, Ghandchi-Tehrani M, Li E. Adaptive feedforward control design for gust loads alleviation and lco suppression. In: 29th Congress of the International Council of the Aeronautical Sciences, St. Petersburg, Russia, 2014.

[17] Ghandchi Tehrani M, Da Ronch A. Gust load alleviation using nonlinear feedforward control. In: EURODYN IX International Conference on Structural Dynamics, Porto, Portugal, 2014.

[18] Da Ronch A, Tantaroudas ND, Jiffri S, Mottershead JE. A nonlinear controller for flutter suppression: from simulation to wind tunnel testing. In: 55 th AIAA/ASMe/ASCE/AHS/SC Structures, Structural Dynamics, and Materials Conference, National Harbor, Maryland, 2014.

[19] Papatheou E, Tantaroudas ND, Da Ronch A, Cooper JE, Mottershead JE. Active 
control for flutter suppression: an experimental investigation. In: International Forum on Aeroelasticity and Structural Dynamics, Bristol, UK, 2013.

[20] Truitt A, Mahmoodi SN. A review on active wind energy harvesting designs. Int J Precis Eng Man 2013; 9 (14): 1667-1675.

[21] Zhao W, Païdoussis MP, Tang L, Liu M, Jiang J. Theoretical and experimental investigations of the dynamics of cantilevered flexible plates subjected to axial flow. J Sound Vib 2012; 3 (331): 575-587.

[22] Chad Gibbs S, Wang I, Dowell E. Theory and experiment for flutter of a rectangular plate with a fixed leading edge in three-dimensional axial flow. J Fluid Struct 2012; (34): 68-83.

[23] Tang L, Pa1 Doussis MP. On the instability and the post-critical behaviour of twodimensional cantilevered flexible plates in axial flow. J Sound Vib 2007; (305): 97115.

[24] Tang DM, Yamamoto H, H. Dowell E. Flutter and limit cycle oscillations of twodimensional panels in three-dimensional axial flow. J Fluid Struct 2003; 2 (17): $225-242$.

[25] Tang D, Dowell EH. Limit cycle oscillations of two-dimensional panels in low subsonic flow. Int J Nonlin Mech 2002; 7 (37): 1199-1209.

[26] Xia Y, Michelin S, Doare O. Fluid-solid-electric lock-in of energy-harvesting piezoelectric flags. Physical Review Applied 2015; (3): 14009. 
[27] Dunn P, John D. Nonlinear stall flutter and divergence analysis of cantilevered graphite/epoxy wings. AIAA J 1992; 1 (30): 153-162.

[28] Tang D, Dowell EH, Hall KC. Limit cycle oscillations of a cantilevered wing in low subsonic flow. AIAA J 1999; 3 (37): 364-371.

[29] Li J, Hong S, Gu S, Choi Y, Nakhmanson S, Heinonen O, Karpeev D, No K. Polymer piezoelectric energy harvesters for low wind speed. Appl Phys Lett 2014; 1 (104): 12902.

[30] Fernandes AC, Rostami AB. Hydrokinetic energy harvesting by an innovative vertical axis current turbine. Renew Energy 2015; (81):694-706.

[31] Abdelkefi A, Hajj MR, Nayfeh AH. Phenomena and modeling of piezoelectric energy harvesting from freely oscillating cylinders. Nonlinear Dynam 2012; 2 (70): $1377-1388$.

[32] Bibo A, Alhadidi AH, Daqaq MF. Exploiting a nonlinear restoring force to improve the performance of flow energy harvesters. J Appl Phys 2015; 4 (117): 45103.

[33] Abdelkefi A, Hajj MR, Nayfeh AH. Piezoelectric energy harvesting from transverse galloping of bluff bodies. Smart Mater Struct 2013; 1 (22): 15014.

[34] Bryant M, Garcia E. Development of an aeroelastic vibration power harvester. In: Proceedings of SPIE Active and Passive Smart Structures and Integrated Systems, San Diego, California, 2009. 
[35] Zhao YH, Hu HY. Aeroelastic analysis of a non-linear airfoil based on unsteady vortex lattice model. J Sound Vib 2004; (276): 491-510.

[36] Dowell EH, Thomas JP, Hall KC. Transonic limit cycle oscillation analysis using reduced order aerodynamic models. J Fluid Struct 2004; 1 (19): 17-27.

[37] Lee BHK, Jiang LY, Wong YS. Flutter of an airfoil with a cubic restoring force. J Fluid Struct 1999; 1 (13): 75-101.

[38] Lee BHK, Price SJ, Wong YS. Nonlinear aeroelastic analysis of airfoils: bifurcation and chaos. Prog Aerosp Sci 1999; 3 (35): 205-334.

[39] Platzer M, Carta FO. AGARD manual on aeroelasticity in axial-flow turbomachines: structural dynamics and aeroelasticity. North Atlantic Treaty Organization, Advisory Group for Aerospace Research and Development, 1988.

[40] Akaydın HD, Elvin N, Andreopoulos Y. Wake of a cylinder: a paradigm for energy harvesting with piezoelectric materials. Exp Fluids 2010; 1 (49): 291-304.

[41] Jung H, Seung-Woo L. The experimental validation of a new energy harvesting system based on the wake galloping phenomenon. Smart Mater Struct 2011; (20): 55022.

[42] Tantaroudas ND, Da Ronch A, Nonlinear Reduced Order Aeroservoelastic Analysis of Very Flexible Aircraft, In Marques P and Da Ronch A (Eds.), Novel Concepts in Unmanned Aircraft Aerodynamics, Flight Stability, and Control. John Wiley \& Sons Inc, Dec 2016. ISBN: 1118928687. 
[43] Silva TMP, De Marqui Jr. C. Passive and hybrid piezoelectric circuits to reduce induced-atmospheric turbulence vibration of a plate-like wing. In: Proceedings of SPIE Active and Passive Smart Structures and Integrated Systems, San Diego, California, 2013.

[44] Shang JS. Three decades of accomplishments in computational fluid dynamics. Prog Aerosp Sci 2004; 3 (40): 173-197.

[45] Mittal R, Iaccarino G. Immersed boundary methods. Annu Rev Fluid Mech 2005; (37): 239-261.

[46] Ghoreyshi M, Cummings RM, Da Ronch A, Badcock KJ. Transonic aerodynamic load modeling of X-31 aircraft pitching motions. AIAA J 2013; 10 (51): 24472464.

[47] Vallespin D, Badcock KJ, Da Ronch A, White MD, Perfect P, Ghoreyshi M. Computational fluid dynamics framework for aerodynamic model assessment. Prog Aerosp Sci 2012; (52): 2-18.

[48] Da Ronch A, Vallespin D, Ghoreyshi M, Badcock KJ. Evaluation of dynamic derivatives using computational fluid dynamics. AIAA J 2012; 2 (50): 470-484.

[49] Da Ronch A, Ghoreyshi M, Badcock KJ. On the generation of flight dynamics aerodynamic tables by computational fluid dynamics. Prog Aerosp Sci 2011; 8 (47): 597-620.

[50] Ghoreyshi M, Badcock KJ, Da Ronch A, Marques S, Swift A, Ames N. 
Framework for establishing limits of tabular aerodynamic models for flight dynamics analysis. J Aircraft 2011; 1 (48): 42-55.

[51] Mehmood A, Abdelkefi A, Hajj MR, Nayfeh AH, Akhtar I, Nuhait AO. Piezoelectric energy harvesting from vortex-induced vibrations of circular cylinder. J Sound Vib 2013; 19 (332): 4656-4667.

[52] Akcabay DT, Young YL. Hydroelastic response and energy harvesting potential of flexible piezoelectric beams in viscous flow. Phys Fluids 2012; 5 (24): 54106.

[53] Xiang J, Wu Y, Li D. Energy harvesting from the discrete gust response of a piezoaeroelastic wing: modeling and performance evaluation. J Sound Vib 2015; (343): 176-193.

[54] Abdelkefi A, Nuhait AO. Modeling and performance analysis of cambered wingbased piezoaeroelastic energy harvesters. Smart Mater Struct 2013; 9 (22): 95029.

[55] Tang L, Païdoussis MP, Jiang J. Cantilevered flexible plates in axial flow: energy transfer and the concept of flutter-mill. J Sound Vib 2009; (326): 263-276.

[56] Abdelkefi A, Ghommem M, Nuhait AO, Hajj MR. Nonlinear analysis and enhancement of wing-based piezoaeroelastic energy harvesters. J Sound Vib 2014; 1 (333): 166-177.

[57] Dunnmon JA, Stanton SC, Mann BP, Dowell EH. Power extraction from aeroelastic limit cycle oscillations. J Fluid Struct 2011; 8 (27): 1182-1198.

[58] Bryant M, Garcia E. Modeling and testing of a novel aeroelastic flutter energy 
harvester. J Vib Acoust 2011; 1 (133): 11010.

[59] Zhu J, Zhang W. Coupled analysis of multi-impact energy harvesting from lowfrequency wind induced vibrations. Smart Mater Struct 2015; 4 (24): 45007.

[60] Mccracken A, Da Ronch A, Timme S, Badcock KJ. Solution of linear systems in fourier-based methods for aircraft applications. Int J Comput Fluid D 2013; 2 (27): 79-87.

[61] Da Ronch A, Mccracken AJ, Badcock KJ, Widhalm M, Campobasso MS. Linear frequency domain and harmonic balance predictions of dynamic derivatives. $\mathbf{J}$ Aircraft 2013; 3 (50): 694-707.

[62] Sirohi J, Mahadik R. Harvesting wind energy using a galloping piezoelectric beam. J Vib Acoust 2012; (134): 110091.

[63] Abdelkefi A, Nayfeh AH, Hajj MR. Design of piezoaeroelastic energy harvesters. Nonlinear Dynam 2012; 4 (68): 519-530.

[64] Barrero-Gil A, Alonso G, Sanz-Andres A. Energy harvesting from transverse galloping. J Sound Vib 2010; 14 (329): 2873-2883.

[65] Caliò R, Rongala U, Camboni D, Milazzo M, Stefanini C, de Petris G, Oddo C. Piezoelectric energy harvesting solutions. Sensors-Basel 2014; 3 (14): 4755-4790.

[66] Cook-Chennault KA, Thambi N, Sastry AM. Powering mems portable devices-a review of non-regenerative and regenerative power supply systems with special emphasis on piezoelectric energy harvesting systems. Smart Mater Struct 2008; 4 
(17): 43001.

[67] Priya S. Advances in energy harvesting using low profile piezoelectric transducers. J Electroceram 2007; 1 (19): 167-184.

[68] Anton SR, Sodano HA. A review of power harvesting using piezoelectric materials (2003-2006). Smart Mater Struct 2007; 3 (16): R1-R21.

[69] Stanton SC, Erturk A, Mann BP, Dowell EH, Inman DJ. Nonlinear nonconservative behavior and modeling of piezoelectric energy harvesters including proof mass effects. J Intel Mat Syst Str 2012; 2 (23): 183-199.

[70] Dai HL, Abdelkefi A, Javed U, Wang L. Modeling and performance of electromagnetic energy harvesting from galloping oscillations. Smart Mater Struct 2015; 4 (24): 45012.

[71] Bender K, Muturi N, Spies A, Lee CL. Aeroelastic energy harvesting using a nonlinear electromagnetic oscillator. In: 54th AIAA/ASME/ASCE/AHS/ASC Structures, Structural Dynamics, and Materials Conference, Boston, Massachusetts, 2013.

[72] Bonisoli E, Manca N, Ghandchi-Tehrani M, Da Ronch A, Gianfrancesco M. Extended frequency bandwidth through multi-degree-of-freedom nonlinear magneto-mechanical energy harvesting. In: International Conference on Structural Engineering Dynamics, Lagos, Portugal, 2015.

[73] Vicente-Ludlam D, Barrero-Gil A, Velazquez A. Optimal electromagnetic energy 
extraction from transverse galloping. J Fluid Struct 2014; (51): 281-291.

[74] Chen J, Dhanushkodi A, Lee CL. Energy harvesting measurements from stall flutter limit cycle oscillations. In: Proceedings of SPIE Active and Passive Smart Structures and Integrated Systems, San Diego, California, 2014.

[75] Michelin S, Doare O. Energy harvesting efficiency of piezoelectric flags in axial flows. J Fluid Mech 2013; (714): 489-504.

[76] De Marqui Jr C, Erturk A, Inman DJ. An electromechanical finite element model for piezoelectric energy harvester plates. J Sound Vib 2009; (327): 9-25.

[77] Yang Y, Zhao L, Tang L. Comparative study of tip cross-sections for efficient galloping energy harvesting. Appl Phys Lett 2013; 6 (102): 64105.

[78] Abdelkefi A, Barsallo N. Comparative modeling of low-frequency piezomagnetoelastic energy harvesters. J Intel Mat Syst Str 2014; 1045389X$14523860 X$.

[79] Abdelkefi A, Yan Z, Hajj MR. Modeling and nonlinear analysis of piezoelectric energy harvesting from transverse galloping. Smart Mater Struct 2013; 2 (22): 25016.

[80] Sirohi J, Mahadik R. Piezoelectric wind energy harvester for low-power sensors. J Intel Mat Syst Str 2011; 1045389X-11428366X.

[81] Xie J, Yang J, Hu H, Hu Y, Chen X. A piezoelectric energy harvester based on flow-induced flexural vibration of a circular cylinder. J Intel Mat Syst Str 2012; 2 
(23): 135-139.

[82] Dai H, Abdelkefi A, Wang L. Theoretical modeling and nonlinear analysis of piezoelectric energy harvesting from vortex-induced vibrations. J Intel Mat Syst Str 2014; 14 (25): 1861-1874.

[83] Zhao L, Tang L, Yang Y. Synchronized charge extraction in galloping piezoelectric energy harvesting. J Intel Mat Syst Str 2015; (in press).

[84] Tang L, Zhao L, Yang Y, Lefeuvre E. Equivalent circuit representation and analysis of galloping-based wind energy harvesting. IEEE/ASME Transactions on Mechatronics 2015; 2 (20): 834-844.

[85] Erturk A, Vieira W, De Marqui Jr C, Inman DJ. On the energy harvesting potential of piezoaeroelastic systems. Appl Phys Lett 2010; 18 (96): 184103.

[86] Dias JAC, De Marqui Jr C, Erturk A. Hybrid piezoelectric-inductive flow energy harvesting and dimensionless electroaeroelastic analysis for scaling. Appl Phys Lett 2013; 4 (102): 44101.

[87] Dias JAC, De Marqui Jr C, Erturk A. Three-degree-of-freedom hybrid piezoelectric-inductive aeroelastic energy harvester exploiting a control surface. AIAA J 2014; 2 (53): 394-404.

[88] Bae J, Inman DJ. Aeroelastic characteristics of linear and nonlinear piezoaeroelastic energy harvester. J Intel Mat Syst Str 2014; 25 (4): 401-416.

[89] Kwon S. A T-shaped piezoelectric cantilever for fluid energy harvesting. Appl 
Phys Lett 2010; 97 (16): 16410216.

[90] Ewere F, Wang G, Cain B. Experimental investigation of galloping piezoelectric energy harvesters with square bluff bodies. Smart Mater Struct 2014; 10 (23): 104012.

[91] Bryant M, Mahtani RL, Garcia E. Wake synergies enhance performance in aeroelastic vibration energy harvesting. J Intel Mat Syst Str 2012; 10 (23): 11311141.

[92] Zhao D, Ega E. Energy harvesting from self-sustained aeroelastic limit cycle oscillations of rectangular wings. Appl Phys Lett 2014; 10 (105): 103903.

[93] Abdelkefi A, Hajj MR. Performance enhancement of wing-based piezoaeroelastic energy harvesting through free-play nonlinearity. Theor Appl Mech Lett 2013; 4 (3): 41001.

[94] Giacomello A, Porfiri M. Underwater energy harvesting from a heavy flag hosting ionic polymer metal composites. J Appl Phys 2011; 8 (109): 84903.

[95] Shukla S, Govardhan RN, Arakeri JH. Flow over a cylinder with a hinged-splitter plate. J Fluid Struct 2009; 4 (25): 713-720.

[96] Akaydin HD, Elvin N, Andreopoulos Y. The performance of a self-excited fluidic energy harvester. Smart Mater Struct 2012; 2 (21): 25007.

[97] Doaré O, Michelin S. Piezoelectric coupling in energy-harvesting fluttering flexible plates: linear stability analysis and conversion efficiency. J Fluid Struct 2011; 8 
(27): $1357-1375$.

[98] Piñeirua M, Doaré O, Michelin S. Influence and optimization of the electrodes position in a piezoelectric energy harvesting flag. J Sound Vib 2015; (346): 200215.

[99] Li S, Yuan J, Lipson H. Ambient wind energy harvesting using cross-flow fluttering. J Appl Phys 2011; 2 (109): 26104.

[100] Hobeck JD, Inman DJ. Dual cantilever flutter: Experimentally validated lumped parameter modeling and numerical characterization. J Fluids Struct 2016, 61: 324338.

[101] Bernitsas MM, Raghavan K, Ben-Simon Y, Garcia EM. VIVACE (vortex induced vibration aquatic clean energy): a new concept in generation of clean and renewable energy from fluid flow. J Offshore Mech Arct 2008; 4 (130): 41101.

[102] Abdelkefi A, Yan Z, Hajj MR. Power generation from galloping-based piezoaeroelastic energy harvesters for different cross-section geometries. In: 54th AIAA/ASME/ASCE/AHS/ASC Structures, Structural Dynamics, and Materials Conference, Boston, Massachusetts, 2013.

[103] Sivadas V, Wickenheiser AM. Small-scale wind energy harvesting from flowinduced vibrations. In: ASME 2011 Conference on Smart Materials, Adaptive Structures and Intelligent Systems, Scottsdale, Arizona, 2011.

[104] Ali M, Arafa M, Elaraby M. Harvesting energy from galloping oscillations. In: 
World Congress on Engineering, London, UK, 2013.

[105] Meseguer J, Sanz-Andrés A, Alonso G. Determination of maximum mechanical energy efficiency in energy galloping systems. J Eng Mech 2014; 4014101.

[106] Ibarra D, Sorribes F, Alonso G, Meseguer J. Transverse galloping of twodimensional bodies having a rhombic cross-section. J Sound Vib 2014; 13 (333): $2855-2865$.

[107] Zhao L, Tang L, Yang Y. Comparison of modeling methods and parametric study for a piezoelectric wind energy harvester. Smart Mater Struct 2013; 12 (22): 125003.

[108] Bibo A, Daqaq MF. On the optimal performance and universal design curves of galloping energy harvesters. Appl Phys Lett 2014; 2 (104): 23901.

[109] Abdelkefi A, Yan Z, Hajj MR. Temperature impact on the performance of galloping-based piezoaeroelastic energy harvesters. Smart Mater Struct 2013; 5 (22): 55026.

[110] Yan Z, Abdelkefi A, Hajj MR. Piezoelectric energy harvesting from hybrid vibrations. Smart Mater Struct 2014; 2 (23): 25026.

[111] Zhao L, Tang L, Yang Y. Enhanced piezoelectric galloping energy harvesting using 2 degree-of- freedom cut-out cantilever with magnetic interaction. Jpn J Appl Phys 2014; (53): 60302.

[112] Vicente-Ludlam D, Barrero-Gil A, Velazquez A. Enhanced mechanical energy 
extraction from transverse galloping using a dual mass system. J Sound Vib 2015; (339): 290-303.

[113] Rostami AB, Fernandes AC. The effect of inertia and flap on autorotation applied for hydrokinetic energy harvesting. Appl Energy 2015; (143):312-323.

[114] Xiao Q, Zhu Q. A review on flow energy harvesters based on flapping foils. J Fluid Struct 2014; (46): 174-191.

[115] Wu Y, Li D, Xiang J. Performance analysis and parametric design of an airfoilbased piezoaeroelastic energy harvester. In: 56th AIAA/ASCE/AHS/ASC Structures, Structural Dynamics, and Materials Conference, Kissimmee, Florida, 2015.

[116] Bryant M, Garcia E. Energy harvesting: a key to wireless sensor nodes. In: 2nd International Conference on Smart Materials and Nanotechnology in Engineering, Weihai, China, 2009.

[117] Bryant M, Wolff E, Garcia E. Aeroelastic flutter energy harvester design: the sensitivity of the driving instability to system parameters. Smart Mater Struct 2011; (20): 125017.

[118] Bryant M, Schlichting AD, Garcia E. Toward efficient aeroelastic energy harvesting: device performance comparisons and improvements through synchronized switching. In: Proceedings of SPIE Active and Passive Smart Structures and Integrated Systems, San Diego, California, 2013. 
[119] Erturk A, Inman DJ. Piezoelectric power generation for civil infrastructure systems. In: Nondestructive Characterization for Composite Materials, Aerospace Engineering, Civil Infrastructure, and Homeland Security, San Diego, California, 2011.

[120] Abdelkefi A, Nayfeh AH, Hajj MR. Enhancement of power harvesting from piezoaeroelastic systems. Nonlinear Dynam 2012; 4 (68): 531-541.

[121] Abdelkefi A, Nayfeh AH, Hajj MR. Modeling and analysis of piezoaeroelastic energy harvesters. Nonlinear Dynam 2012; 2 (67): 925-939.

[122] Abdelkefi A, Hajj MR, Nayfeh AH. Uncertainty quantification of piezoelectric energy harvesters from aeroelastic vibrations. In: MATEC Web of Conferences, Marrakech, Morocco, 2012.

[123] De Marqui Jr C, Anicezio MDM, Vieira WGR, Tristao SF. Linear and nonlinear modeling and experiments of a piezoaeroelastic energy harvester. In: 3rd Annual Meeting of the ASME/AIAA Smart Materials, Adaptive Structures, and Intelligent Systems Conference, Philadelphia, Pennsylvania, 2010.

[124] Sousa VC, de M Anicézio M, De Marqui Jr C, Erturk A. Enhanced aeroelastic energy harvesting by exploiting combined nonlinearities: theory and experiment. Smart Mater Struct 2011; 9 (20): 94007.

[125] Anicezio MDM, Erturk A, De Marqui Jr C, Inman DJ. Linear and nonlinear aeroelastic energy harvesting using electromagnetic induction. In: ASME 
Conference on Smart Materials, Adaptive Structures and Intelligent Systems, Scottsdale, Arizona, 2011.

[126] Mahtani RL, Garcia E. Wake effects and synergies in aeroelastic wind power harvesting systems. In: 50th AIAA Aerospace Sciences Meeting including the New Horizons Forum and Aerospace Exposition, Nashville, Tennessee, 2012.

[127] Bryant M, Tse R, Garcia E. Investigation of host structure compliance in aeroelastic energy harvesting. In: ASME Conference on Smart Materials, Adaptive Structures and Intelligent Systems, Stone Mountain, Georgia, 2012.

[128] Bibo A, Daqaq MF. Energy harvesting under combined aerodynamic and base excitations. J Sound Vib 2013; 20 (332): 5086-5102.

[129] De Marqui Jr C, Erturk A. Electroaeroelastic analysis of airfoil-based wind energy harvesting using piezoelectric transduction and electromagnetic induction. J Intel Mat Syst Str 2013; 7SI (24): 846-854.

[130] Zhao L, Yang Y. Enhanced aeroelastic energy harvesting with a beam stiffener. Smart Mater Struct 2015; 3 (24): 32001.

[131] Allen JJ, Smits AJ. Energy harvesting eel. J Fluid Struct 2001; 3 (15): 629-640.

[132] Pobering S, Ebermeyer S, Schwesinger N. Generation of electrical energy using short piezoelectric cantilevers in flowing media. In: Proceedings of SPIE Active and Passive Smart Structures and Integrated Systems, San Diego, California, 2009.

[133] Pobering S, Schwesinger N. A novel hydropower harvesting device. In: 
International Conference on MEMs, NANO \& Smart Systems, Banff, Alberta, 2004.

[134] Perez M, Boisseau S, Gasnier P, Willemin J, Reboud JL. An electret-based aeroelastic flutter energy harvester. Smart Mater Struct 2015; 3 (24): 35004.

[135] Sivadas V, Wickenheiser AM. A study of several vortex-induced vibration techniques for piezoelectric wind energy harvesting. In: Proceedings of SPIE Active and Passive Smart Structures and Integrated Systems, San Diego, California, 2011.

[136] Goushcha O, Elvin N, Andreopoulos Y. Interactions of vortices with a flexible beam with applications in fluidic energy harvesting. Appl Phys Lett 2014; 2 (104): 21919.

[137] Abdelkefi A, Scanlon JM, Mcdowell E, Hajj MR. Performance enhancement of piezoelectric energy harvesters from wake galloping. Appl Phys Lett 2013; 3 (103): 33903.

[138] Abdelkefi A, Hasanyan A, Montgomery J, Hall D, Hajj MR. Incident flow effects on the performance of piezoelectric energy harvesters from galloping vibrations. Theoretical and Applied Mechanics Letters 2014; 2 (4): 22002.

[139] Kitio Kwuimy CA, Litak G, Borowiec M, Nataraj C. Performance of a piezoelectric energy harvester driven by air flow. Appl Phys Lett 2012; 2 (100): 24103. 
[140] Goushcha O, Akaydin HD, Elvin N, Andreopoulos Y. Energy harvesting prospects in turbulent boundary layers by using piezoelectric transduction. J Fluid Struct 2015; (54): 823-847.

[141] J W Sohn SBCA. An investigation on piezoelectric energy harvesting for mems power sources. Proceedings of the Institution of Mechanical Engineers, Part C: Journal of Mechanical Engineering Science 2005; 7 (219): 429.

[142] Wang D, Chang KH. Electromagnetic energy harvesting from flow induced vibration. Microelectron J 2010; 6 (41): 356-364.

[143] Wang D, Liu N. A shear mode piezoelectric energy harvester based on a pressurized water flow. Sensors and Actuators A: Physical 2011; 2 (167): 449-458.

[144] Vatansever D, Hadimani RL, Shah T, Siores E. An investigation of energy harvesting from renewable sources with PVDF and PZT. Smart Mater Struct 2011; (20): 55019.

[145] Fei F, Mai JD, Li WJ. A wind-flutter energy converter for powering wireless sensors. Sensor Actuat A-Phys 2012; 1 (173): 163-171.

[146] Clair DS, Bibo A, Sennakesavababu VR, Daqaq MF, Li G. A scalable concept for micropower generation using flow-induced self-excited oscillations. Appl Phys Lett 2010; 14 (96): 144103.

[147] Bibo A, Li G, Daqaq MF. Electromechanical modeling and normal form analysis of an aeroelastic micro-power generator. J Intel Mat Syst Str 2011; 6 (22): 577- 
592.

[148] Erturk A, Bilgen O, Fontenille M, Inman DJ. Piezoelectric energy harvesting from macro-fiber composites with an application to morphing-wing aircrafts. In: Proceedings of the 19th International Conference on Adaptive Structures and Technologies, Ascona, Switzerland, 2008.

[149] De Marqui Jr C, Erturk A, Inman DJ. Piezoaeroelastically coupled modeling and analysis of electrical power generation and shunt damping for a cantilever plate. In: Proceedings of the 17th International Conference on Composite Materials, Edinburgh, UK, 2009.

[150] De Marqui Jr C, Erturk A, Inman DJ. Effect of segmented electrodes on piezoelastic and piezo-aero-elastic responses of generator plates. In: Proceedings of the ASME 2009 Conference on Smart Materials, Adaptive Structures and Intelligent Systems, Oxnard, California, 2009.

[151] De Marqui Jr C, Erturk A, Inman DJ. Piezoaeroelastic modeling and analysis of a generator wing with continuous and segmented electrodes. J Intel Mat Syst Str 2010; 10 (21): 983-993.

[152] De Marqui Jr C, Vieira WG, Erturk A, Inman DJ. Modeling and analysis of piezoelectric energy harvesting from aeroelastic vibrations using the doublet-lattice method. J Vib Acoust 2011; 1 (133): 11003.

[153] Vieira WGR, Nitzsche F, De Marqui Jr C. Non-linear modeling and analysis of 
composite helicopter blade for piezoelectric energy harvesting. In: Proceedings of the ASME 2012 Conference on Smart Materials, Adaptive Structures and Intelligent Systems, Stone Mountain, Georgia, 2012.

[154] Abdelkefi A, Ghommem M. Piezoelectric energy harvesting from morphing wing motions for micro air vehicles. Theoretical and Applied Mechanics Letters 2013; 5 (3): 52004.

[155] Wu Y, Li D, Xiang J. Design and analysis of energy harvester based on aeroelastic vibration. In: the Ninth International Conference on Vibration Engineering and Technology of Machinery, Nanjing, 2013.

[156] Pozzi M, Guo S, Zhu M. Harvesting energy from the dynamic deformation of an aircraft wing under gust loading. In: Proceedings of SPIE Health Monitoring of Structural and Biological Systems, Smart Structures/NDE, San Diego, California, 2012.

[157] Pozzi M, Canziani A, Durazo-Cardenas I, Zhu M. Experimental characterisation of macro fibre composites and monolithic piezoelectric transducers for strain energy harvesting. In: Proceedings of SPIE Health Monitoring of Structural and Biological Systems, Smart Structures/NDE, San Diego, California, 2012.

[158] Magoteaux KC, Sanders B, Sodano HA. Investigation of an energy harvesting small unmanned air vehicle. In: Proceedings of SPIE Active and Passive Smart Structures and Integrated Systems, San Diego, California, 2008. 
[159] Anton SR, Inman DJ. Vibration energy harvesting for unmanned aerial vehicles. In: Proceedings of SPIE Active and Passive Smart Structures and Integrated Systems, San Diego, California, 2008.

[160] Anton SR, Erturk A, Inman DJ. Multifunctional self-charging structures using piezoceramics and thin-film batteries. Smart Mater Struct 2010; (19): 115021.

[161] Anton SR, Erturk A, Kong N, Ha DS, Inman DJ. Self-charging structures using piezoceramics and thin-film batteries. In: ASME Conference on Smart Materials, Adaptive Structures and Intelligent Systems, 2009.

[162] Anton SR, Erturk A, Inman DJ. Piezoelectric energy harvesting from multifunctional wing spars for UAVs-part2: experiments and storage applications. In: SPIE Smart Structures and Materials+ Nondestructive Evaluation and Health Monitoring, 2009.

[163] Erturk A, Anton SR, Inman DJ. Piezoelectric energy harvesting from multifunctional wing spars for UAVs-part 1: coupled modeling and preliminary analysis. In: SPIE's 16th Annual International Symposium on Smart Structures and Materials \& Nondestructive Evaluation and Health Monitoring, 2009.

[164] Anton SR, Inman DJ. Performance modeling of unmanned aerial vehicles with on-board energy harvesting. In: Proceedings of SPIE Active and Passive Smart Structures and Integrated Systems, San Diego, California, 2011.

[165] Erturk A, Renno JM, Inman DJ. Modeling of piezoelectric energy harvesting from 
an L-shaped beam-mass structure with an application to UAVs. J Intel Mat Syst Str 2009;

[166] Gilbert JM, Balouchi F. Comparison of energy harvesting systems for wireless sensor networks. Int J Automation Computing 2008; 4 (5): 334-347.

[167] Mathúna CÓ, O Donnell T, Martinez-Catala RV, Rohan J, O Flynn B. Energy scavenging for long-term deployable wireless sensor networks. Talanta 2008; 3 (75): 613-623.

[168] Seah WK, Eu ZA, Tan H. Wireless sensor networks powered by ambient energy harvesting (WSN-HEAP)-survey and challenges. In: 1st International Conference on Wireless Communication, Vehicular Technology, Information Theory and Aerospace \& Electronic Systems Technology, Aalborg, Denmark, 2009.

[169] Boragno C, Festa R, Mazzino A. Elastically bounded flapping wing for energy harvesting. Appl Phys Lett 2012; 25 (100): 253906.

[170] Roundy S, Wright PK, Rabaey J. A study of low level vibrations as a power source for wireless sensor nodes. Comput Commun 2003; 11 (26): 1131-1144.

[171] Zhu M, Worthington E, Worthington E. Design and testing of piezoelectric energy harvesting devices for generation of higher electric power for wireless sensor networks. In: 8th Annual IEEE Conference on Sensors, Christchurch, New Zealand, 2009.

[172] Arms SW, Townsend CP, Churchill DL, Galbreath JH, Mundell SW. Power 
management for energy harvesting wireless sensors. In: Smart Structures and Materials: Smart Electronics, MEMS, BioMEMS, and Nanotechnology, Bellingham, Washington, 2005.

[173] Churchill DL, Hamel MJ, Townsend CP, Arms SW. Strain energy harvesting for wireless sensor networks. In: Smart Structures and Materials: Smart Electronics, MEMS, BioMEMS, and Nanotechnology, San Diego, California, 2003.

[174] Kansal A, Srivastava MB. An environmental energy harvesting framework for sensor networks. In: International Symposium on Low Power Electronics and Design, Seoul, Korea, 2003.

[175] Vigorito CM, Ganesan D, Barto AG. Adaptive control of duty cycling in energyharvesting wireless sensor networks. In: 4th Annual IEEE Communications Society Conference on Sensor, Mesh and Ad Hoc Communications and Networks, San Diego, California, 2007.

[176] Alippi C, Galperti C. An adaptive system for optimal solar energy harvesting in wireless sensor network nodes. IEEE Transactions on Circuits and Systems I: Regular Papers 2008; 6 (55): 1742-1750.

[177] Park C, Chou PH. Ambimax: autonomous energy harvesting platform for multisupply wireless sensor nodes. In: 3rd Annual IEEE Communications Society on Sensor and Ad Hoc Communications and Networks, Reston, Virginia, 2006.

[178] Niyato D, Hossain E, Rashid MM, Bhargava VK. Wireless sensor networks with 
energy harvesting technologies: a game-theoretic approach to optimal energy management. Wireless Communications, IEEE 2007; 4 (14): 90-96.

[179] Marsic V, Giuliano A, Pozzi M, Zhu M, Williams S. Performance testing of a low power consumption wireless sensor communication system integrated with an energy harvesting power source. In: The Third International Conference on Sensor Device Technologies and Applications, 2012.

[180] Di Monaco F, Ghandchi Tehrani M, Elliott SJ, Bonisoli E, Tornincasa S. Energy harvesting using semi-active control. J Sound Vib 2013; 23 (332): 6033-6043.

[181] Afsharfard A, Farshidianfar A. Application of single unit impact dampers to harvest energy and suppress vibrations. J Intel Mat Syst Str 2014; 1045389X$14535012 X$.

[182] Wang Y, Inman DJ. A survey of control strategies for simultaneous vibration suppression and energy harvesting via piezoceramics. J Intel Mat Syst Str 2012; 18 (23): 2021-2037.

[183] Wang Y, Inman DJ. Simultaneous energy harvesting and gust alleviation for a multifunctional composite wing spar using reduced energy control via piezoceramics. J Compos Mater 2013; 1 (47): 125-146.

[184] Bryant M, Pizzonia M, Mehallow M, Garcia E. Energy harvesting for selfpowered aero-structure actuation. In: Proceedings of SPIE Active and Passive Smart Structures and Integrated Systems, San Diego, California, 2014. 


\section{Figure Captions}

Fig. 1 Various energy harvesting sources that can be used to generate electric energy [3].

Fig. 2 Schematic of LCO of a plate in axial flow.

Fig. 3 Predicted LCO response as function of the flow velocity for a "flapping flag"

[25]. ((a) RMS tip amplitude (b) frequency)

Fig. 4 Schematic of a cantilevered plate subjected to uniform flow.

Fig. 5 Predicted transverse displacement of a cylinder VIV under different load resistances in the EH circuit; from Ref. [31], C Springer, with permission.

Fig. 6 Inner circulation responsible for galloping phenomena [32].

Fig. 7 Analytical (red dashed line) and numerical (black line) predictions of the transverse displacement of a bluff body with an isosceles triangle section undergoing galloping oscillations [33]. (C) IOP Publishing. Reproduced with permission. All rights reserved)

Fig. 8 Schematic of a typical 2DOF wing section.

Fig. 9 Dependency of predicted LCO amplitudes of a typical wing section with reduced speed [38]. ((a) dimensionless plunge amplitude (b) pitch amplitude)

Fig. 10 Dependency of predicted LCO frequency of a typical wing section with reduced velocity [38].

Fig. 11 Near wake of a cylinder $\left(\operatorname{Re}=8 \times 10^{4}\right)$; from Ref. [40], (C) Springer, with 
permission.

Fig. 12 Schematic of EH from vortex-induced vibration.

Fig. 13 Amplitude versus frequency of the VIV for a downstream beam from an experiment; from Ref. [40], (C) Springer, with permission.

Fig. 14 Schematic of wake galloping phenomena.

Fig. 15 Typical velocity-amplitude curves of four aerodynamic instability phenomena

[41]. (C IOP Publishing. Reproduced with permission. All rights reserved) ((a) VIV (b)

galloping and flutter (c) wake galloping (d) buffeting)

Fig. 16 In (a), atmospheric turbulence signal generated numerically and filtered by von Kármán and Dryden spectrum (with turbulence scale 350 m and RMS turbulence velocity $0.5 \mathrm{~m} \mathrm{~s}^{-1}$ ); and in (b), frequency domain response of a plate-like wing (with piezoelectric actuator) to the turbulence (SC: short-circuit; OC: open-circuit; RLs: resistive-inductive series; p: passive; a: active); from [43], C SPIE, with permission. Fig. 17 Schematics of the operation modes for a piezoelectric material [68]; in (a), 33mode, and in (b) 31-mode. ((a) 33-mode, and (b) 31-mode)

Fig. 18 Common piezoelectric transducers; from Ref. [67], (C) Springer, with permission.

((a) Thunder (b) Active Fiber Composite (c) Macro Fiber Composite (d) Radial Field Diaphragm (e) QuickPack (f) Bimorp)

Fig. 19 Schematic representation of piezoelectric EH; from Ref. [67], (c) Springer, with 
permission.

Fig. 20 Schematic of (a) a bluff body in cross-flow linked to an electromagnetic generator [70] (C) IOP Publishing. Reproduced with permission. All rights reserved) and (b) electromagnetic inductor energy harvester device in [71].

Fig. 21 (a) Schematic of magneto-mechanical energy harvester and (b) corresponding prototypes in [72].

Fig. 22 Schematic of (a) a slender flexible plate flapping in a uniform axial flow and (b) 2D flapping of the plate; from Ref. [75], (C) Cambridge University Press, with permission.

Fig. 23 Galloping energy harvester with tip body having equilateral triangle cross section [79]. (C) IOP Publishing. Reproduced with permission. All rights reserved) Fig. 24 Schematic of the hybrid piezoelectric-inductive energy harvester [86]. Fig. 25 (a) Schematic of T-shape cantilever energy harvester and (b) experimental setup in the wind tunnel [89].

Fig. 26 Galloping piezoelectric energy harvester: (a) prototype, (b) prototype in wind tunnel test section [90]. (C) IOP Publishing. Reproduced with permission. All rights reserved)

Fig. 27 In (a), two aeroelastic energy harvesters mounted in a wind tunnel test section in tandem configuration; in (b), overhead schematic view of the smoke wire flow 
visualization setup used to image the wake of the energy harvesters [91].

Fig. 28 In (a), experimental setup of an energy harvesting platform in a wind tunnel; and in (b), two configurations of the piezoelectric generators [92].

Fig. 29 Schematic of (a) the experimental setup and (b) the pitch free-play mechanism [93]. Reprinted with permission as per http://creativecommons.org/licenses/by-nc$\mathrm{nd} / 4.0 /$

Fig. 30 Schematic of the experimental setup used to measure the harvesting capabilities of an IPMC mounted on a heavy host flag [94]. IPMC denotes ionic polymer metal composites.

Fig. 31 Schematic of a cylinder with a hinged-rigid splitter plate. The rigid splitter plate was free to rotate about the hinge at the base of the cylinder [95].

Fig. 32 Experimental setup of plate-based EH in axial flow [57].

Fig. 33 Schematic of the flexible heavy flag flapping in a uniform water stream comprising the host structure and ionic polymer metal composites strip [94].

Fig. 34 Schematic of different flapping leaf configurations [29].

Fig. 35 A schematic of the dual cantilever EH system [100].

Fig. 36 Schematic of EH from galloping oscillations; from Ref. [101], (c ASME, with permission.

Fig. 37 Different cross sections of bluff bodies. ((a) cylinder b) triangle c) pentagon d) 
D-shaped e) square)

Fig. 38 In (a) top and side views of the self-excited harvester, and in (b) electrode configuration for patches [96]. (C IOP Publishing. Reproduced with permission. All rights reserved)

Fig. 39 Schematics of 2DOF EH from galloping oscillations by (a) Zhao et al. [111] (C) 2014 by The Japan Society of Applied Physics, reprinted with permission) and (b) Vicente-Ludlam et al. [112].

Fig. 40 Schematic of an aerofoil-based EH system [115].

Fig. 41 Aerofoil-based EH; from Ref. [116], (C) SPIE, with permission.

Fig. 42 Schematic of modified aerofoil-based EH devices; in (a) from [87], and in (b) from [130]. (C IOP Publishing. Reproduced with permission. All rights reserved) Fig. 43 Schematic of an EH ell [131].

Fig. 44 Schematic of EH from wake galloping phenomena [41]. (C) IOP Publishing. Reproduced with permission. All rights reserved)

Fig. 45 Schematic of EH based on variable flow via a membrane [142].

Fig. 46 Schematic of EH based on blown airflow within a cavity [146].

Fig. 47 Schematic of a wing-like plate; from Ref. [152], (C) ASME, with permission.

Fig. 48 Schematic of the piezoelectric wing spar [53].

Fig. 49 Remote control aircraft with modifications; from [159], (C) SPIE, with 
permission.

Fig. 50 Schematic of the multifunctional wing spar; from Ref. [161], (C) ASME, with permission.

Fig. 51 Photograph and schematic of Tyndall $25 \mathrm{~mm}$ wireless sensor module [167].

Fig. 52 Generic WSN node with EH devices; from Ref. [166], (C) Springer, with permission.

Fig. 53 Schematic of strain EH system; from Ref. [173], (C SPIE, with permission. Fig. 54 Concept of multifunctional composite wing spar [183].

Fig. 55 In (a), dimensionless tip displacement frequency response function of multifunctional composite wing spar, and in (b) harvested power spectrum at one optimal load resistance identified in [183].

Fig. 56 Wind tunnel apparatus for demonstrating tab actuation powered by aerofoilbased harvester; from Ref. [184], C SPIE, with permission.

Fig. 57 Apparatus for placing the aeroelastic energy harvester at an angle of attack in the flow; from Ref. [184], (C) SPIE, with permission. 
Tables

Table 1 Power density of several EH methods [5].

\begin{tabular}{|l|c|}
\hline \multicolumn{1}{|c|}{ Harvesting technology } & Power density \\
\hline Acoustic noise $(100 \mathrm{~dB})$ & $9.60 \times 10^{-7} \mathrm{~W} / \mathrm{cm}^{3}$ \\
\hline Thermoelectric $\left(10^{\circ} \mathrm{C}\right.$ gradient) & $4.00 \times 10^{-5} \mathrm{~W} / \mathrm{cm}^{3}$ \\
\hline Vibration (small microwave oven) & $1.16 \times 10^{-4} \mathrm{~W} / \mathrm{cm}^{3}$ \\
\hline Piezoelectric (shoe inserts) & $3.30 \times 10^{-4} \mathrm{~W} / \mathrm{cm}^{3}$ \\
\hline Solar cells (outdoors at noon) & $1.50 \times 10^{-2} \mathrm{~W} / \mathrm{cm}^{2}$ \\
\hline
\end{tabular}


Table 2 Comparison of several EH designs and implementations.

\begin{tabular}{|c|c|c|c|c|c|}
\hline Year & Dimension $^{1}$ & Transducer & Average Power Output & Reference & Information \\
\hline \multicolumn{6}{|c|}{ Flapping Flags } \\
\hline 2009 & $\begin{array}{l}232 \mathrm{~cm} \times \\
200 \mathrm{~cm}\end{array}$ & $\begin{array}{l}\text { Electromagnetic } \\
\text { induction }\end{array}$ & $5 \mathrm{~W}\left(9.25 \mathrm{~m} \mathrm{~s}^{-1}\right)$ & {$[55]$} & Nonlinear beam and UVLM \\
\hline 2011 & $\begin{array}{l}71 \mathrm{~mm} \times \\
6.7 \mathrm{~mm}\end{array}$ & $\begin{array}{l}\text { Ionic polymer } \\
\text { metal } \\
\text { composites } \\
\text { (IPMC) }\end{array}$ & $1 \mathrm{nW}\left(0.6-1.1 \mathrm{~m} \mathrm{~s}^{-1}\right)$ & [94] & Water tunnel \\
\hline 2011 & $\begin{array}{l}0.31 \mathrm{~cm} \times \\
0.101 \mathrm{~cm}\end{array}$ & $\begin{array}{l}\text { Piezoelectric } \\
\text { effect (PZT) }\end{array}$ & $2.5 \mathrm{~mW}\left(27 \mathrm{~m} \mathrm{~s}^{-1}\right)$ & [57] & $\begin{array}{l}\text { Nonlinear beam and UVLM; Wind } \\
\text { tunnel }\end{array}$ \\
\hline \multicolumn{6}{|c|}{ Flapping Leaves } \\
\hline
\end{tabular}




\begin{tabular}{|c|c|c|c|c|c|}
\hline 2014 & $\begin{array}{l}7.5 \mathrm{~cm} \times \\
2 \mathrm{~cm}\end{array}$ & $\begin{array}{l}\text { Piezoelectric } \\
\text { effect (PDVF) }\end{array}$ & $0.98 \mu \mathrm{W}\left(3.9 \mathrm{~m} \mathrm{~s}^{-1}\right)$ & [29] & Wind tunnel \\
\hline 2014 & $\begin{array}{l}251.6 \mathrm{~mm} \times \\
100.6 \mathrm{~mm}\end{array}$ & $\begin{array}{l}\text { Piezoelectric } \\
\text { effect (PZT) }\end{array}$ & $55 \mathrm{~mW}\left(16 \mathrm{~m} \mathrm{~s}^{-1}\right)$ & {$[92]$} & $\begin{array}{l}\text { Wind tunnel; Piezoelectric beam was } \\
\text { connected to host structure }\end{array}$ \\
\hline \multicolumn{6}{|c|}{ VIV of Bluff Bodies } \\
\hline 2011 & $\begin{array}{l}\text { Cylinder } \\
40 \mathrm{~cm} \times \\
1 \mathrm{~cm}\end{array}$ & $\begin{array}{l}\text { Piezoelectric } \\
\text { effect (PZT) }\end{array}$ & $1 \mathrm{~mW}\left(5 \mathrm{~m} \mathrm{~s}^{-1}\right)$ & {$[81]$} & $\begin{array}{l}\text { Linear beam; Deformation of bluff } \\
\text { body was used instead of rigid } \\
\text { displacement }\end{array}$ \\
\hline 2014 & $\begin{array}{l}\text { Cylinder } \\
203 \mathrm{~mm} \times \\
19.8 \mathrm{~mm}\end{array}$ & $\begin{array}{l}\text { Piezoelectric } \\
\text { effect (PZT) }\end{array}$ & $0.1007 \mathrm{~mW}\left(1.20 \mathrm{~m} \mathrm{~s}^{-1}\right)$ & {$[82]$} & $\begin{array}{l}\text { Nonlinear beam and van der Pol } \\
\text { oscillator }\end{array}$ \\
\hline 2015 & Cylinder & Piezoelectric & $1.26 \mathrm{~mW}\left(3.5 \mathrm{~m} \mathrm{~s}^{-1}\right)$ & {$[130]$} & Linear beam model and quasi-steady \\
\hline
\end{tabular}




\begin{tabular}{|c|c|c|c|c|c|}
\hline & $\begin{array}{l}\text { not given } \times \\
\text { not given }\end{array}$ & effect (PZT) & & & $\begin{array}{l}\text { aerodyanmc force; Beam stiffer was } \\
\text { used to enhance power output }\end{array}$ \\
\hline \multicolumn{6}{|c|}{ Galloping of Bluff Bodies } \\
\hline 2010 & $\begin{array}{l}\text { T-shape } \\
60 \mathrm{~mm} \times \\
30 \mathrm{~mm}\end{array}$ & $\begin{array}{l}\text { Piezoelectric } \\
\text { effect (PZT) }\end{array}$ & $2.0 \mathrm{~mW}\left(4 \mathrm{~m} \mathrm{~s}^{-1}\right)$ & [89] & Wind tunnel \\
\hline 2011 & $\begin{array}{l}\text { Triangular } \\
251 \mathrm{~mm} \times \\
40 \mathrm{~mm}\end{array}$ & $\begin{array}{l}\text { Piezoelectric } \\
\text { effect }(\mathrm{PZT})\end{array}$ & $53 \mathrm{~mW}\left(25.8 \mathrm{~m} \mathrm{~s}^{-1}\right)$ & [80] & $\begin{array}{l}\text { Linear beam and quasi-steady } \\
\text { aerodyanmc model; Wind tunnel }\end{array}$ \\
\hline 2012 & $\begin{array}{l}\text { Cylinder } \\
203 \mathrm{~mm} \times \\
39.6 \mathrm{~mm}\end{array}$ & $\begin{array}{l}\text { Piezoelectric } \\
\text { effect (PZT) }\end{array}$ & $0.1 \mathrm{~mW}\left(1.192 \mathrm{~m} \mathrm{~s}^{-1}\right)$ & [96] & Wind tunnel \\
\hline
\end{tabular}




\begin{tabular}{|c|c|c|c|c|c|}
\hline 2012 & $\begin{array}{l}\text { D-shape } \\
235 \mathrm{~mm} \times \\
25 \mathrm{~mm}\end{array}$ & $\begin{array}{l}\text { Piezoelectric } \\
\text { effect (PZT) }\end{array}$ & $1.14 \mathrm{~mW}\left(23.4 \mathrm{~m} \mathrm{~s}^{-1}\right)$ & {$[62]$} & $\begin{array}{l}\text { Quasi-steady aerodynamic model; Wind } \\
\text { tunnel }\end{array}$ \\
\hline 2013 & $\begin{array}{l}\text { D-shape } \\
250 \mathrm{~mm} \times \\
50 \mathrm{~mm}\end{array}$ & $\begin{array}{l}\text { Electromagnetic } \\
\text { induction }\end{array}$ & $37 \mu \mathrm{W}\left(3.25 \mathrm{~m} \mathrm{~s}^{-1}\right)$ & {$[104]$} & $\begin{array}{l}\text { Wind tunnel; D-shape cross section was } \\
\text { recommended. }\end{array}$ \\
\hline 2013 & $\begin{array}{l}\text { Square } \\
150 \mathrm{~mm} \times \\
40 \mathrm{~mm}\end{array}$ & $\begin{array}{l}\text { Piezoelectric } \\
\text { effect (PZT) }\end{array}$ & $4.2 \mathrm{~mW}\left(2.5 \mathrm{~m} \mathrm{~s}^{-1}\right)$ & [77] & $\begin{array}{l}\text { Quasi-steady aerodynamic model; } \\
\text { Square cross section was recommended. }\end{array}$ \\
\hline 2014 & $\begin{array}{l}\text { Square } \\
100 \mathrm{~mm} \times \\
50 \mathrm{~mm}\end{array}$ & $\begin{array}{l}\text { Piezoelectric } \\
\text { effect (PZT) }\end{array}$ & $6.5 \mathrm{~mW}\left(8 \mathrm{~m} \mathrm{~s}^{-1}\right)$ & [90] & Wind tunnel \\
\hline
\end{tabular}




\begin{tabular}{|c|c|c|c|c|c|}
\hline 2015 & $\begin{array}{l}\text { H-shape } \\
300 \mathrm{~mm} \times \\
50 \mathrm{~mm}\end{array}$ & $\begin{array}{l}\text { Piezoelectric } \\
\text { effect (PZT) }\end{array}$ & $\begin{array}{l}11.77 \mathrm{~mW}\left(10 \mathrm{~m} \mathrm{~s}^{-1}\right) \\
45 \mathrm{~mW}\left(14 \mathrm{~m} \mathrm{~s}^{-1}\right)\end{array}$ & {$[59]$} & Empirical aerodynamic model \\
\hline 2015 & $\begin{array}{l}\text { Square } \\
150 \mathrm{~mm} \times \\
40 \mathrm{~mm}\end{array}$ & $\begin{array}{l}\text { Piezoelectric } \\
\text { effect (PZT) }\end{array}$ & $7.57 \mathrm{~mW}\left(5 \mathrm{~m} \mathrm{~s}^{-1}\right)$ & {$[130]$} & $\begin{array}{l}\text { Quasi-steady aerodyanmc model; Wind } \\
\text { tunnel; Beam stiffer was used to } \\
\text { enhance power output }\end{array}$ \\
\hline \multicolumn{6}{|c|}{ Aerofoil-based } \\
\hline 2009 & $\begin{array}{l}13.6 \mathrm{~cm} \times \\
2.95 \mathrm{~cm}\end{array}$ & $\begin{array}{l}\text { Piezoelectric } \\
\text { effect (PZT) }\end{array}$ & $2.25 \mathrm{~mW}\left(7 \mathrm{~m} \mathrm{~s}^{-1}\right)$ & [116] & $\begin{array}{l}\text { Linear structural and Peters model; } \\
\text { Wind tunnel; Use of aerofoil or plate as } \\
\text { structure were compared. }\end{array}$ \\
\hline 2010 & $\begin{array}{l}0.25 \mathrm{~m} \times \\
0.5 \mathrm{~m}\end{array}$ & $\begin{array}{l}\text { Piezoelectric } \\
\text { effect (PZT) }\end{array}$ & $10.2 \mathrm{~mW}\left(12.5 \mathrm{~m} \mathrm{~s}^{-1}\right)$ & [123] & $\begin{array}{l}\text { Nonlinear structural model with free- } \\
\text { play and ULVM; Wind tunnel }\end{array}$ \\
\hline
\end{tabular}




\begin{tabular}{|c|c|c|c|c|c|}
\hline 2010 & $\begin{array}{l}0.25 \mathrm{~m} \times \\
0.5 \mathrm{~m}\end{array}$ & $\begin{array}{l}\text { Piezoelectric } \\
\text { effect (PZT) }\end{array}$ & $10.7 \mathrm{~mW}\left(9.3 \mathrm{~m} \mathrm{~s}^{-1}\right)$ & [85] & $\begin{array}{l}\text { Linear structural and Theodorsen } \\
\text { model; Wind tunnel }\end{array}$ \\
\hline 2011 & $\begin{array}{l}0.25 \mathrm{~m} \times \\
0.5 \mathrm{~m}\end{array}$ & $\begin{array}{l}\text { Piezoelectric } \\
\text { effect (PZT) }\end{array}$ & $106 \mathrm{~mW}\left(18 \mathrm{~m} \mathrm{~s}^{-1}\right)$ & {$[124]$} & $\begin{array}{l}\text { Nonlinear structural model with } \\
\text { combined nonlinearities and } \\
\text { Theodorsen model; Wind tunnel }\end{array}$ \\
\hline 2012 & $\begin{array}{l}0.27 \mathrm{~m} \times \\
\text { not given }\end{array}$ & $\begin{array}{l}\text { Piezoelectric } \\
\text { effect (PZT) }\end{array}$ & $0.55 \mathrm{~W}\left(17 \mathrm{~m} \mathrm{~s}^{-1}\right)$ & {$[120]$} & $\begin{array}{l}\text { Nonlinear structural model with } \\
\text { hardening cubic stiffness in both pitch } \\
\text { and plunge DOFs and quasi-steady } \\
\text { dynamic stall model }\end{array}$ \\
\hline 2012 & $\begin{array}{l}\text { not given } \times \\
\text { not given }\end{array}$ & $\begin{array}{l}\text { Piezoelectric } \\
\text { effect (PZT) }\end{array}$ & $3.7 \mathrm{~mW}\left(1.7 \mathrm{~m} \mathrm{~s}^{-1}\right)$ & {$[63]$} & $\begin{array}{l}\text { Nonlinear structural model with } \\
\text { hardening cubic stiffness in both pitch } \\
\text { and plunge DOFs and quasi-steady }\end{array}$ \\
\hline
\end{tabular}




\begin{tabular}{|c|c|c|c|c|c|}
\hline & & & & & $\begin{array}{l}\text { dynamic stall model; Feedback control } \\
\text { was used to reduce cut-in speed }\end{array}$ \\
\hline 2013 & $\begin{array}{l}5.9 \mathrm{~cm} \times \\
13.6 \mathrm{~cm}\end{array}$ & $\begin{array}{l}\text { Piezoelectric } \\
\text { effect (PZT) }\end{array}$ & $1.43 \mathrm{~mW}\left(2.4 \mathrm{~m} \mathrm{~s}^{-1}\right)$ & {$[118]$} & $\begin{array}{l}\text { Wind tunnel; Synchronized switch } \\
\text { method was introduced to improve } \\
\text { power output. }\end{array}$ \\
\hline 2013 & $\begin{array}{l}\text { not given } \times \\
\text { not given }\end{array}$ & $\begin{array}{l}\text { Piezoelectric } \\
\text { effect (PZT) }\end{array}$ & $0.25 \mathrm{~mW}\left(12.59 \mathrm{~m} \mathrm{~s}^{-1}\right)$ & [93] & Wind tunnel \\
\hline 2013 & $\begin{array}{l}0.127 \mathrm{~m} \times \\
0.2 \mathrm{~m}\end{array}$ & $\begin{array}{l}\text { Electromagnetic } \\
\text { induction }\end{array}$ & $0.1 \mu \mathrm{W}\left(36.24 \mathrm{~m} \mathrm{~s}^{-1}\right)$ & [71] & Wind tunnel \\
\hline 2014 & $\begin{array}{l}0.27 \mathrm{~m} \times \\
\text { not given }\end{array}$ & $\begin{array}{l}\text { Piezoelectric } \\
\text { effect (PZT) }\end{array}$ & $7 \mathrm{~mW}\left(8 \mathrm{~m} \mathrm{~s}^{-1}\right)$ & {$[56]$} & Nonlinear structural model and UVLM \\
\hline 2014 & $0.127 \mathrm{~m} \times$ & Electromagnetic & $55 \mathrm{~mW}\left(38.58 \mathrm{~m} \mathrm{~s}^{-1}\right)$ & {$[74]$} & Nonlinear structural and Theodorsen \\
\hline
\end{tabular}




\begin{tabular}{|c|c|c|c|c|c|}
\hline & $0.2 \mathrm{~m}$ & induction & & & $\begin{array}{l}\text { model; Wind tunnel; EH under stall } \\
\text { condition was studied. }\end{array}$ \\
\hline 2015 & $\begin{array}{l}0.27 \mathrm{~m} \times \\
1 \mathrm{~m}\end{array}$ & $\begin{array}{l}\text { Piezoelectric } \\
\text { effect (PZT) }\end{array}$ & $75 \mathrm{~mW}\left(12 \mathrm{~m} \mathrm{~s}^{-1}\right)$ & {$[115]$} & $\begin{array}{l}\text { Nonlinear structural and Theodorsen } \\
\text { model; The effect of disturbances was } \\
\text { considered. }\end{array}$ \\
\hline 2015 & $\begin{array}{l}84 \mathrm{~mm} \times \\
52 \mathrm{~mm}\end{array}$ & $\begin{array}{l}\text { Piezoelectric } \\
\text { effect (PZT) }\end{array}$ & $1.28 \mathrm{~mW}\left(5 \mathrm{~m} \mathrm{~s}^{-1}\right)$ & {$[130]$} & $\begin{array}{l}\text { Linear structural model and quasi- } \\
\text { steady dynamic stall model; Beam } \\
\text { stiffer was used to enhance power } \\
\text { output. }\end{array}$ \\
\hline \multicolumn{6}{|c|}{ VIVs in wake vortices } \\
\hline 2004 & $\begin{array}{l}5 \mathrm{~mm} \times \\
3 \mathrm{~mm}\end{array}$ & $\begin{array}{l}\text { Piezoelectric } \\
\text { effect (PZT) }\end{array}$ & $6.81 \mu \mathrm{W}\left(2 \mathrm{~m} \mathrm{~s}^{-1}\right)$ & [133] & Beam model \\
\hline
\end{tabular}




\begin{tabular}{|c|c|c|c|c|c|}
\hline 2009 & $\begin{array}{l}14 \mathrm{~mm} \times \\
11.8 \mathrm{~mm}\end{array}$ & $\begin{array}{l}\text { Piezoelectric } \\
\text { effect (PZT) }\end{array}$ & $0.1 \mathrm{~mW}\left(45 \mathrm{~m} \mathrm{~s}^{-1}\right)$ & {$[132]$} & $\begin{array}{l}\text { Beam model; Wind tunnel and Water } \\
\text { tunnel }\end{array}$ \\
\hline 2011 & $\begin{array}{l}0.04 \mathrm{~m} \times \\
\text { not given }\end{array}$ & $\begin{array}{l}\text { Piezoelectric } \\
\text { effect (PZT) }\end{array}$ & $0.35 \mathrm{~mW}(\mathrm{Re}=900)$ & [135] & $\begin{array}{l}\text { Linear beam model; Triangle and } \\
\text { pentagon bluff body was used instead of } \\
\text { cylinder. }\end{array}$ \\
\hline 2015 & $\begin{array}{l}50 \mathrm{~mm} \times \\
15 \mathrm{~mm}\end{array}$ & $\begin{array}{l}\text { Piezoelectric } \\
\text { effect (PDVF) }\end{array}$ & $\begin{array}{l}481 \mu \mathrm{W}\left(15 \mathrm{~m} \mathrm{~s}^{-1}\right) \\
2.1 \mathrm{~mW}\left(30 \mathrm{~m} \mathrm{~s}^{-1}\right)\end{array}$ & {$[134]$} & Linear beam model \\
\hline \multicolumn{6}{|c|}{ Wake Galloping } \\
\hline 2011 & $\begin{array}{l}850 \mathrm{~cm} \times \\
5 \mathrm{~cm}\end{array}$ & $\begin{array}{l}\text { Electromagnetic } \\
\text { induction }\end{array}$ & $370.4 \mathrm{~mW}\left(4.5 \mathrm{~m} \mathrm{~s}^{-1}\right)$ & [41] & Wind tunnel \\
\hline 2013 & $\begin{array}{l}27.5 \mathrm{~cm} \times \\
1.25 \mathrm{~cm}\end{array}$ & $\begin{array}{l}\text { Piezoelectric } \\
\text { effect (PZT) }\end{array}$ & $50 \mu \mathrm{W}\left(3.05 \mathrm{~m} \mathrm{~s}^{-1}\right)$ & {$[137]$} & Wind tunnel \\
\hline
\end{tabular}




\begin{tabular}{|l|l|l|l|l|l|}
\hline 2014 & $26.76 \mathrm{~cm} \times$ & Piezoelectric & $0.35 \mu \mathrm{W}\left(2 \mathrm{~m} \mathrm{~s}^{-1}\right)$ & {$[138]$} & Wind tunnel \\
& $1.28 \mathrm{~cm}$ & effect (PZT) & & \\
\hline
\end{tabular}

${ }^{1}$ Dimension means, respectively, for beam or plate: area of surface; for bluff body: area of wind exposure; for aerofoil: area of wing section. 


\section{Energy Harvesting Flow-induced Vibrations in Aerospace}

Figures

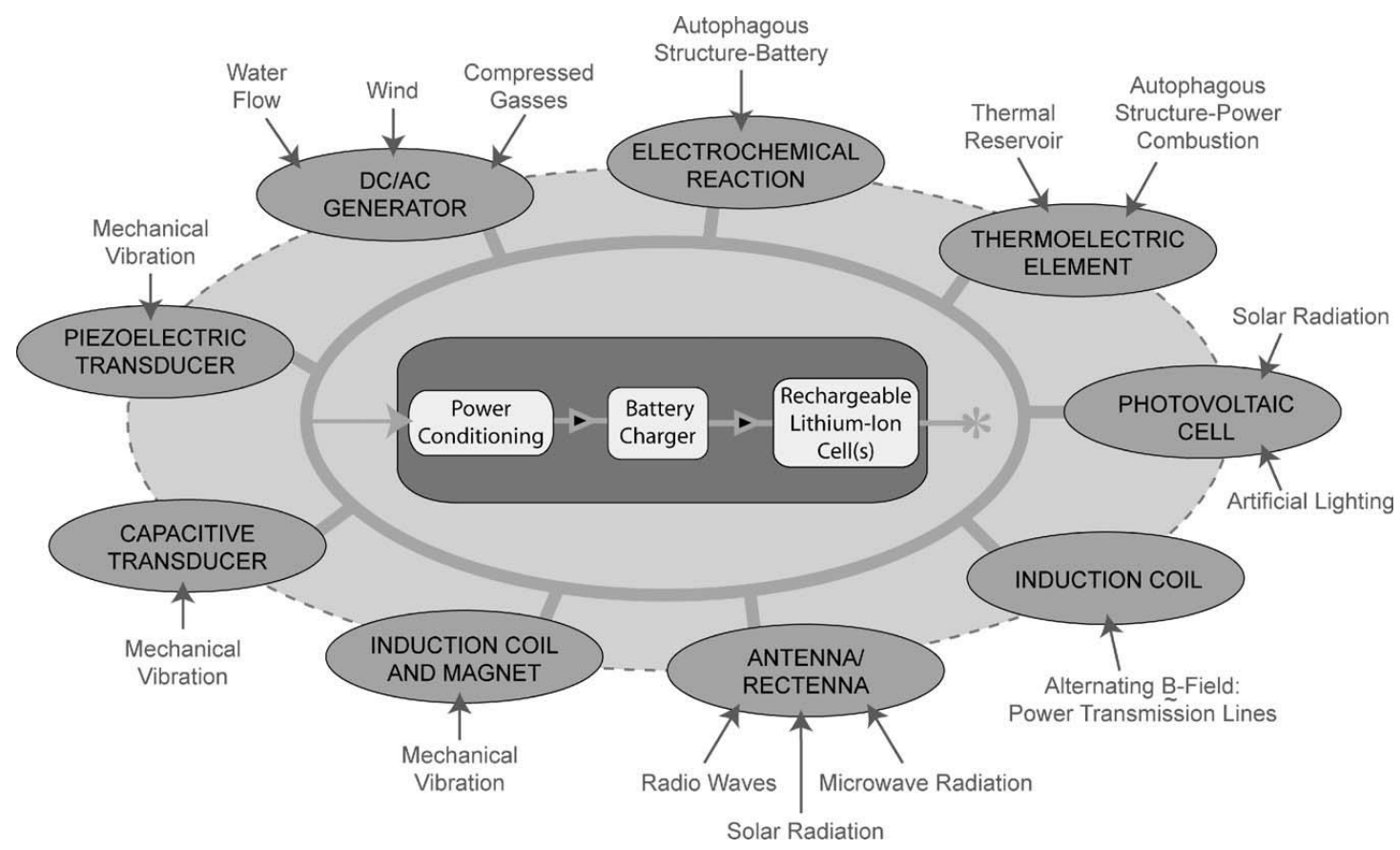

Fig. 1 Various energy harvesting sources that can be used to generate electric energy [3]. 


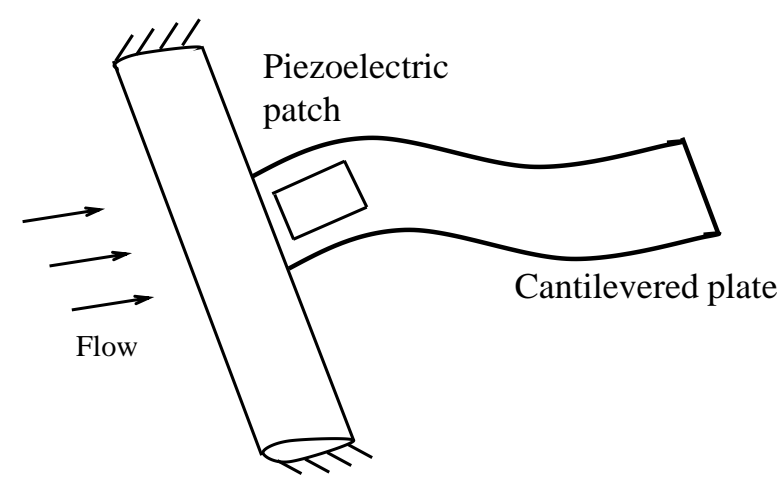

Fig. 2 Schematic of LCO of a plate in axial flow. 


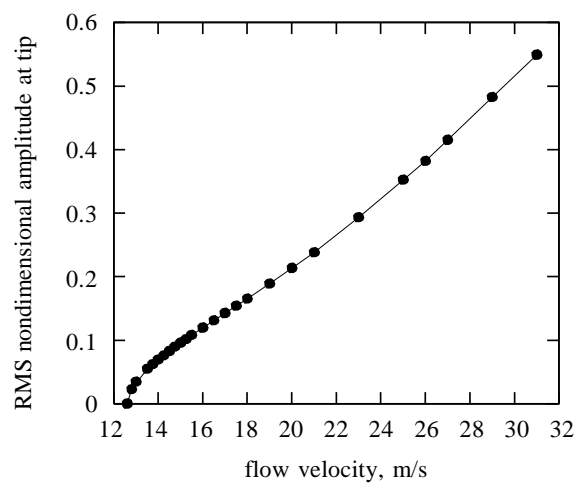

(a) RMS tip amplitude

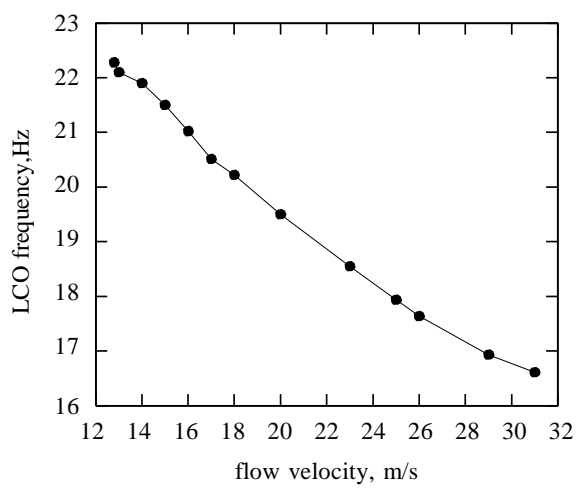

(b) frequency

Fig. 3 Predicted LCO response as function of the flow velocity for a "flapping flag" [25]. 


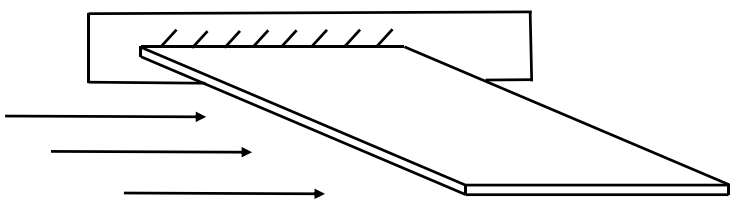

Fig. 4 Schematic of a cantilevered plate subject to uniform flow. 


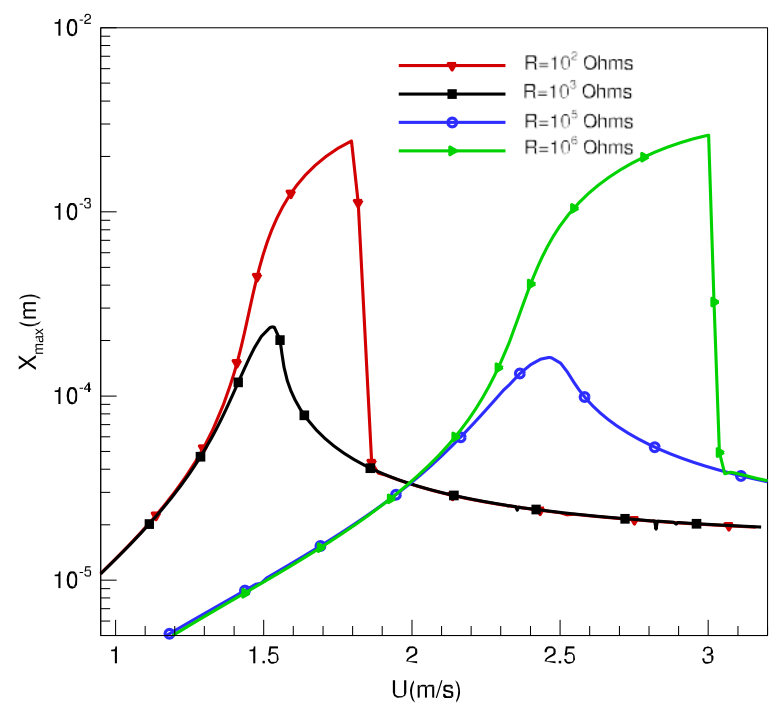

Fig. 5 Predicted transverse displacement of a cylinder VIV under different load resistances in the EH circuit; from Ref. [31], (C) Springer, with permission. 


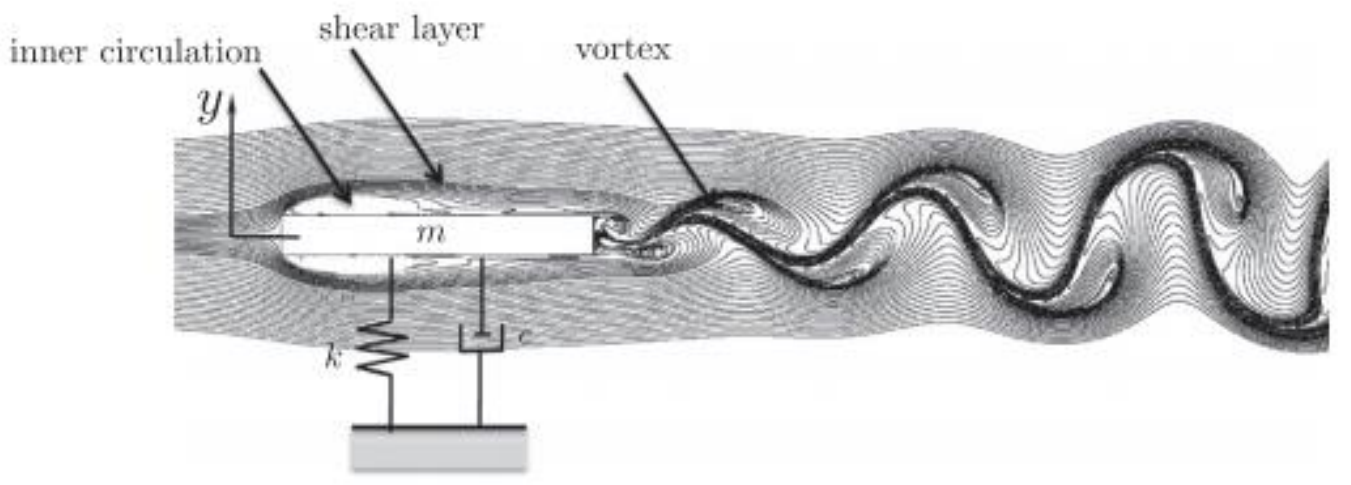

Fig. 6 Inner circulation responsible for galloping phenomena [32]. 


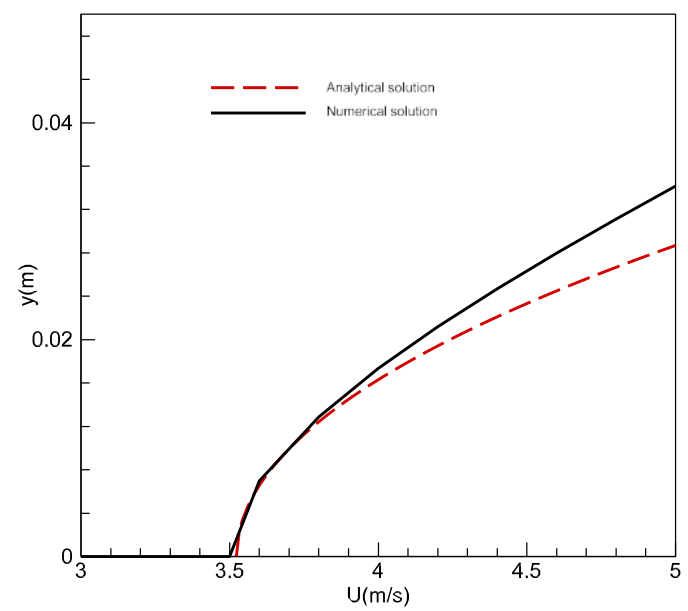

Fig. 7 Analytical (red dashed line) and numerical (black line) predictions of the transverse displacement of a bluff body with an isosceles triangle section undergoing galloping oscillations [33]. (C IOP Publishing. Reproduced with permission. All rights reserved) 


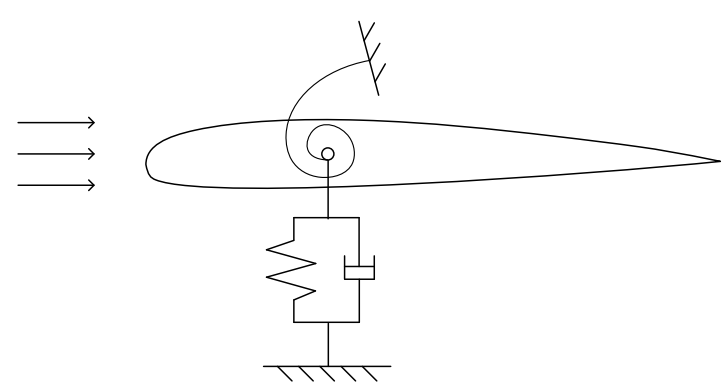

Fig. 8 Schematic of a typical 2DOF wing section. 


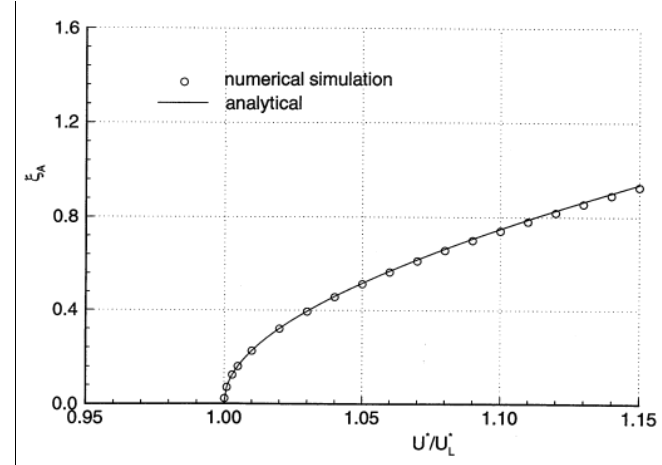

(a) dimensionless plunge amplitude

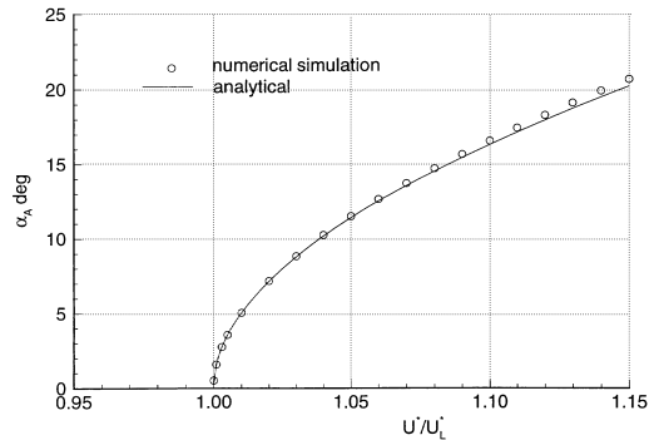

(b) pitch amplitude

Fig. 9 Dependency of predicted LCO amplitudes of a typical wing section with reduced speed [38]. 


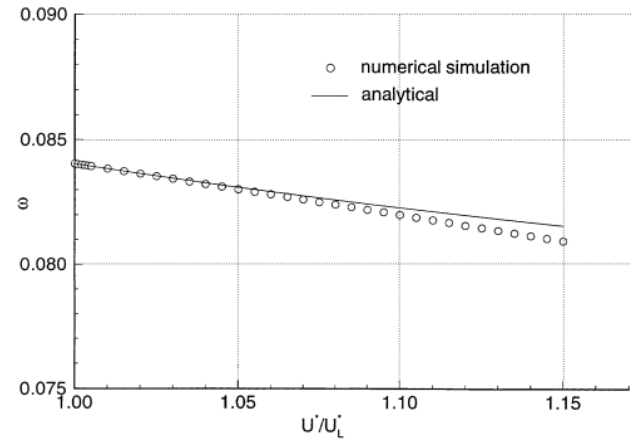

Fig. 10 Dependency of predicted LCO frequency of a typical wing section with reduced velocity [38]. 


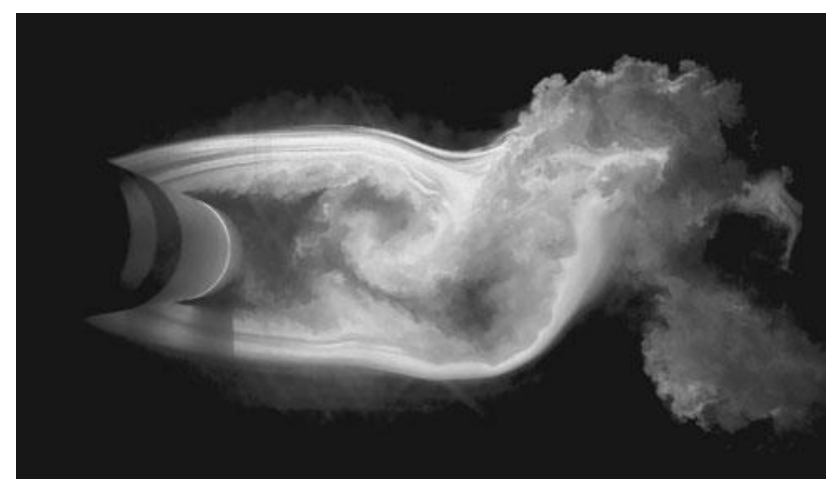

Fig. 11 Near wake of a cylinder $\left(\operatorname{Re}=8 \times 10^{4}\right)$; from Ref. [40], (C) Springer, with permission. 


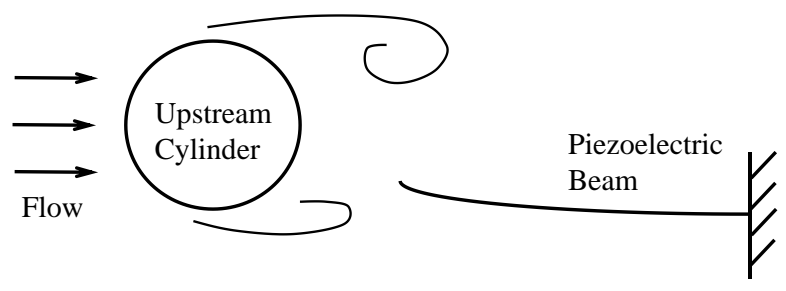

Fig. 12 Schematic of EH from vortex-induced vibration. 


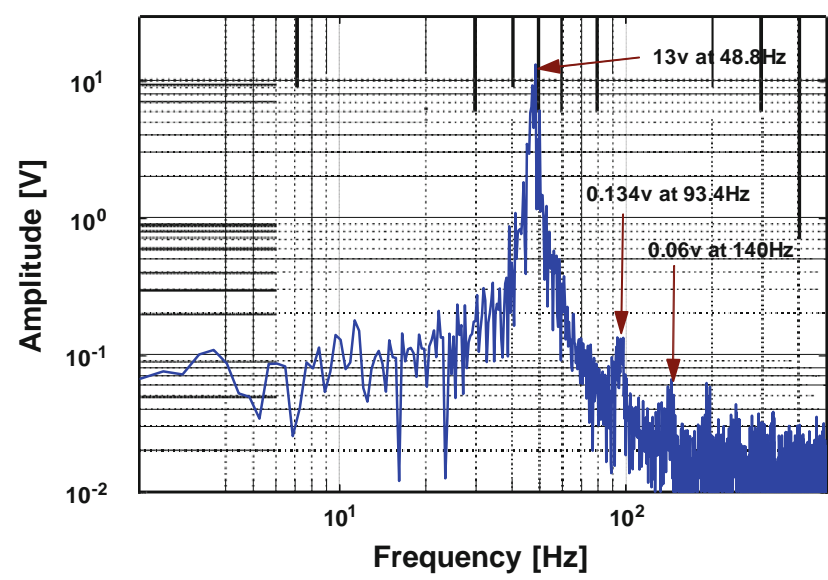

Fig. 13 Amplitude versus frequency of the VIV for a downstream beam from an experiment; from Ref.

[40], (C) Springer, with permission. 


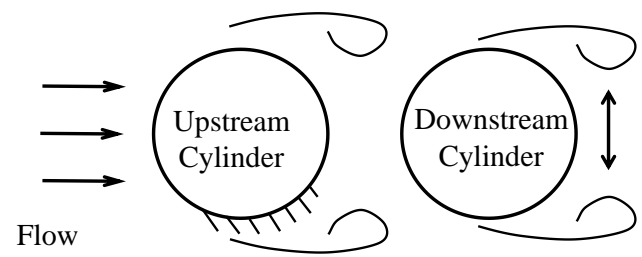

Fig. 14 Schematic of wake galloping phenomena. 


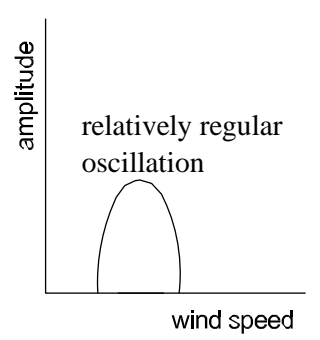

(a) VIV

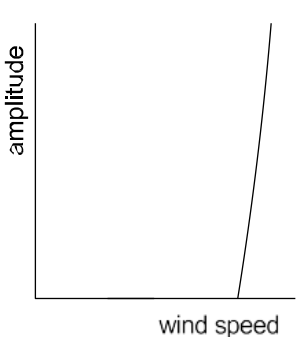

(b) galloping and flutter

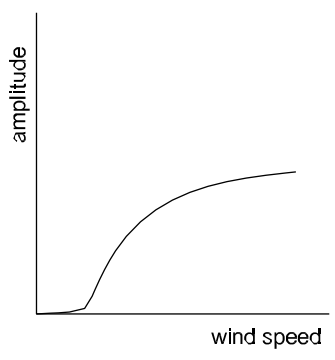

(c) wake galloping

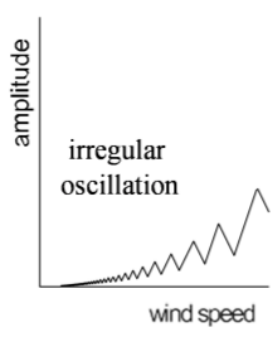

(d) buffeting

Fig. 15 Typical velocity-amplitude curves of four aerodynamic instability phenomena [41]. (@) IOP

Publishing. Reproduced with permission. All rights reserved) 
(a)
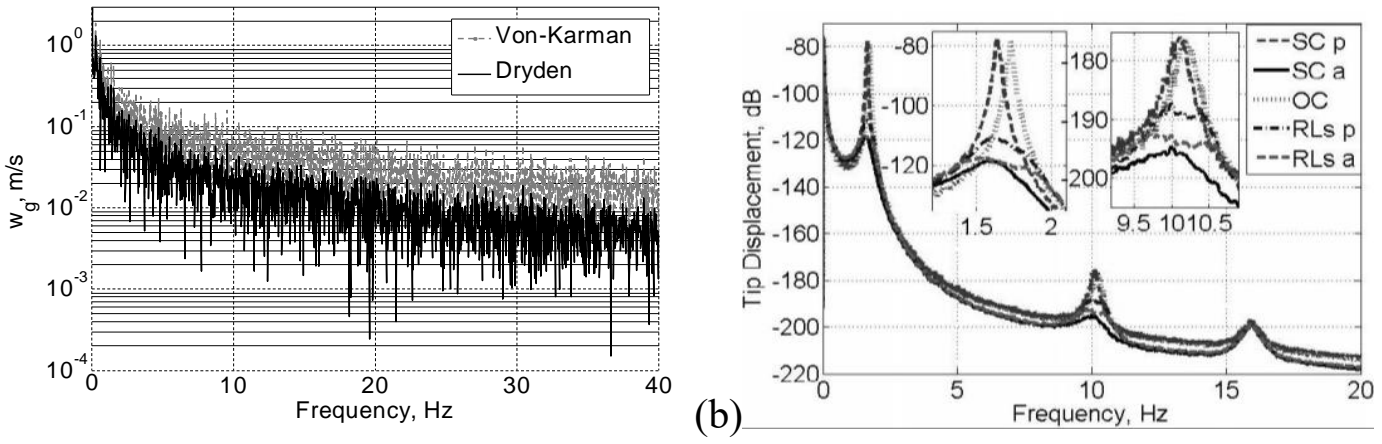

Fig. 16 In (a), atmospheric turbulence signal generated numerically and filtered by von Kármán and

Dryden spectrum (with turbulence scale $350 \mathrm{~m}$ and RMS turbulence velocity $0.5 \mathrm{~m} \mathrm{~s}^{-1}$ ); and in (b),

frequency domain response of a plate-like wing (with piezoelectric actuator) to the turbulence (SC:

short-circuit; OC: open-circuit; RLs: resistive-inductive series; p: passive; a: active); from [43], (C SPIE,

with permission. 


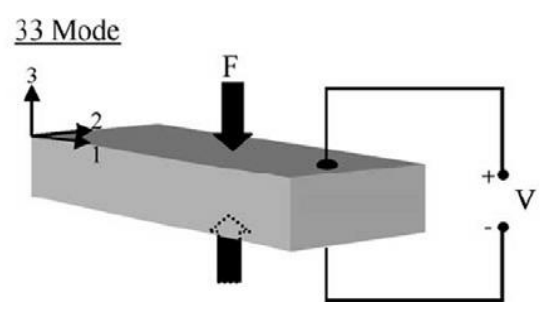

(a) 33-mode $\underline{31 \text { Mode }}$

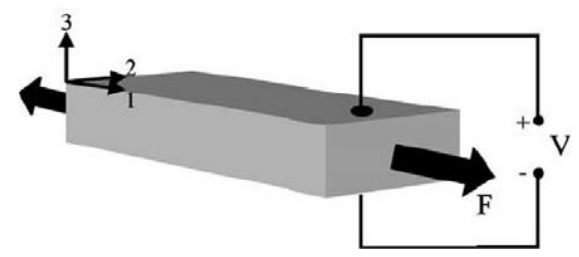

(b) 31-mode

Fig. 17 Schematics of the operation modes for a piezoelectric material [68]; in (a), 33-mode, and in (b)

31-mode. 


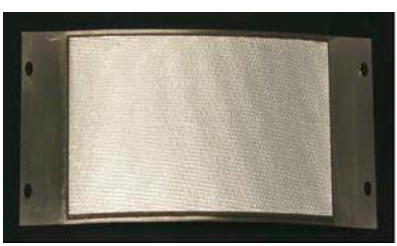

(a) Thunder

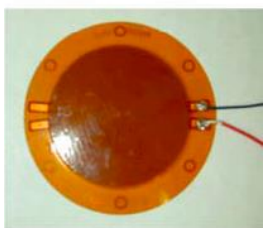

(d) Radial Field Diaphragm

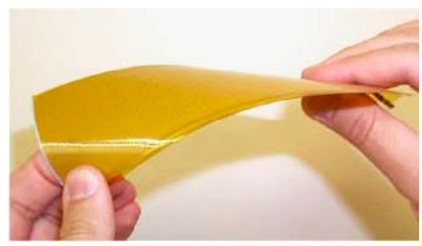

(b) Active Fiber Composite

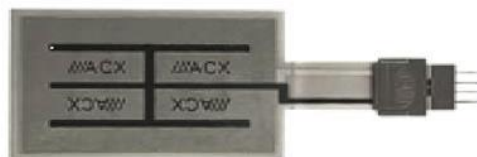

(e) QuickPack (c) Macro Fiber Composite
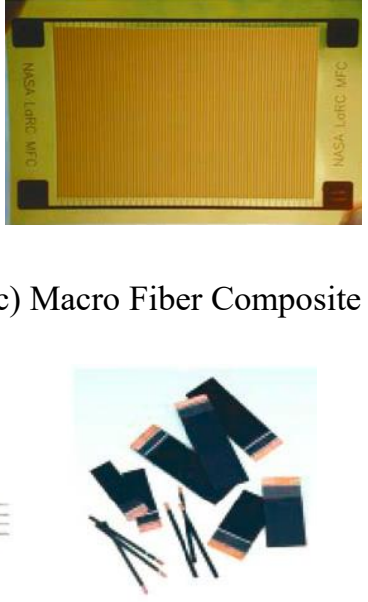

(f) Bimorp

Fig. 18 Common piezoelectric transducers; from Ref. [67], (C) Springer, with permission. 


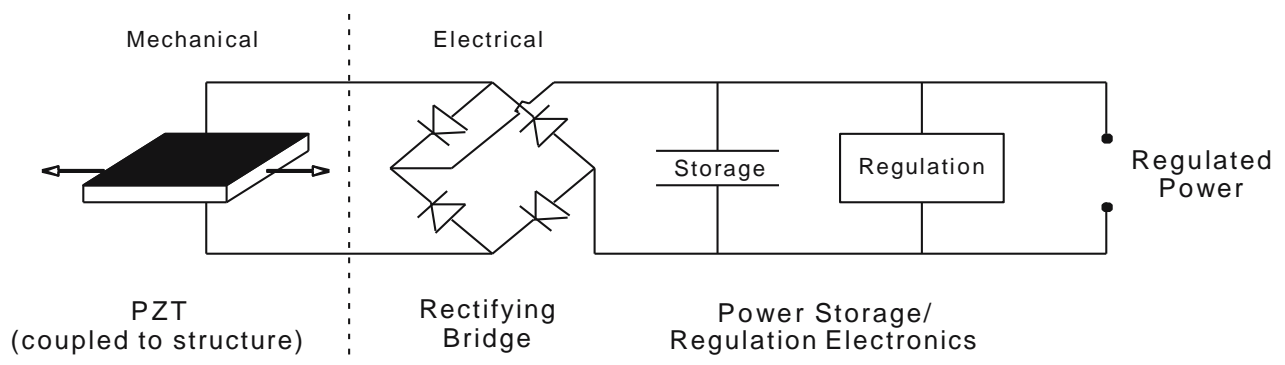

Fig. 19 Schematic representation of piezoelectric EH; from Ref. [67], @ Springer, with permission. 

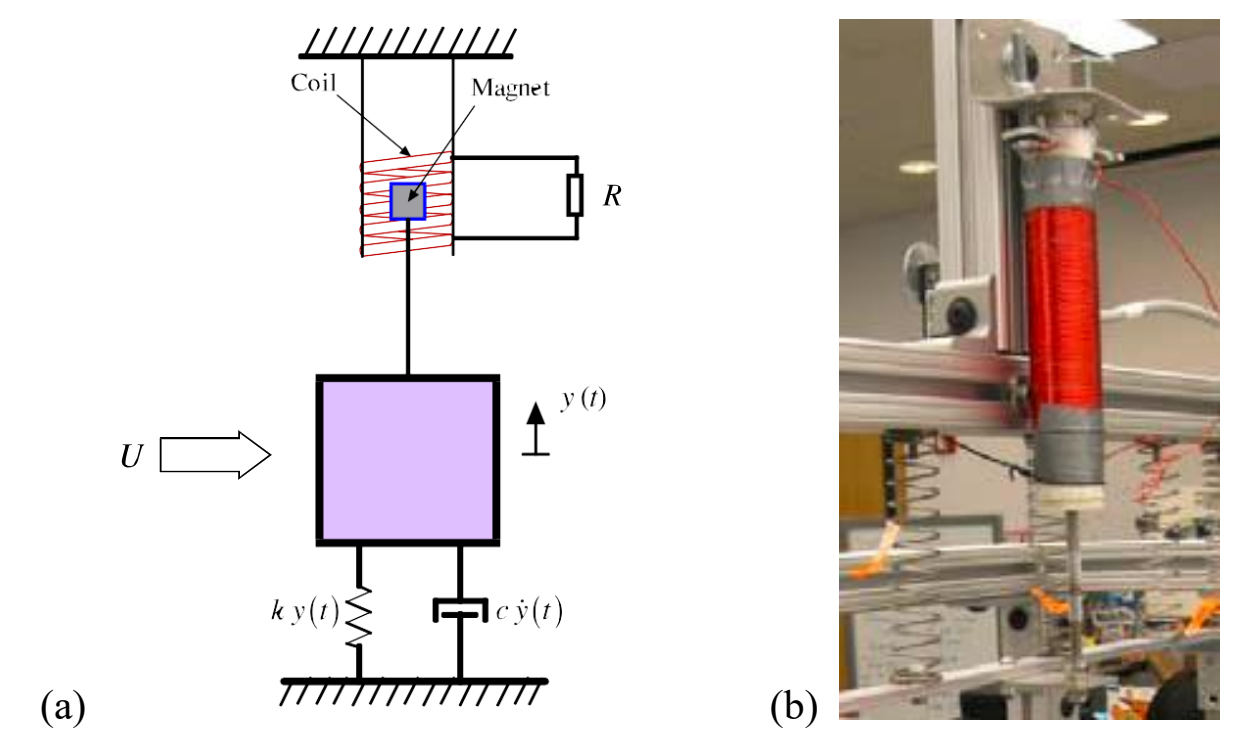

Fig. 20 Schematic of (a) a bluff body in cross-flow linked to an electromagnetic generator [70] (C IOP

Publishing. Reproduced with permission. All rights reserved) and (b) electromagnetic inductor energy

harvester device in [71]. 
(a)

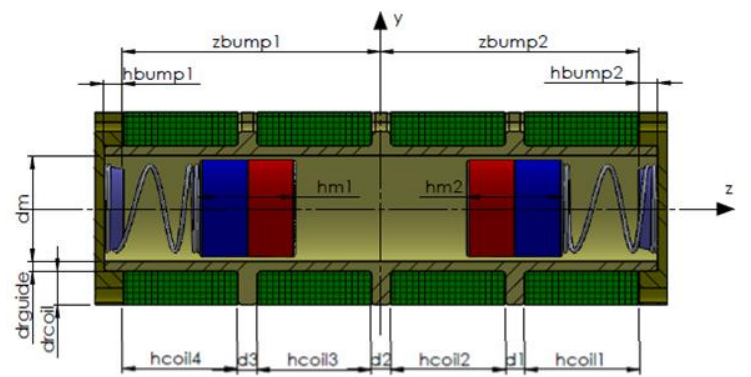

(b)

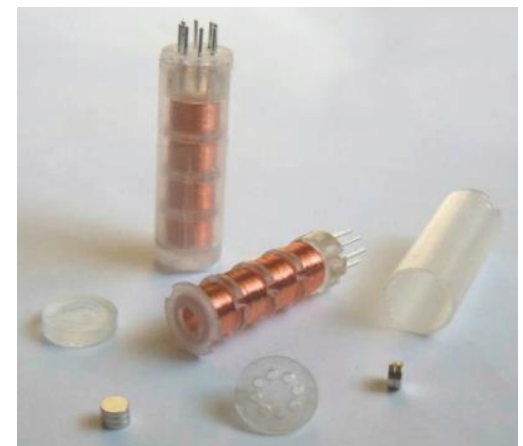

Fig. 21 (a) Schematic of magneto-mechanical energy harvester and (b) corresponding prototypes in [72]. 
(a)

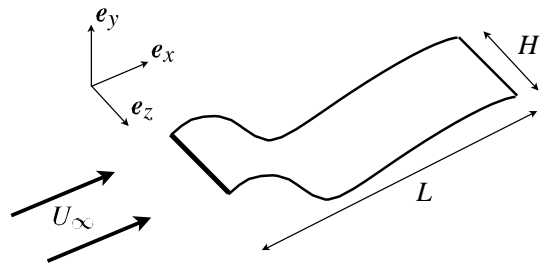

(b)

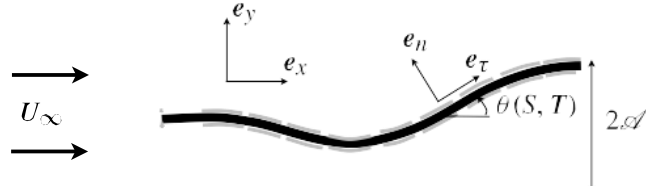

Fig. 22 Schematic of (a) a slender flexible plate flapping in a uniform axial flow and (b) 2D flapping of the plate; from Ref. [75], (C) Cambridge University Press, with permission. 


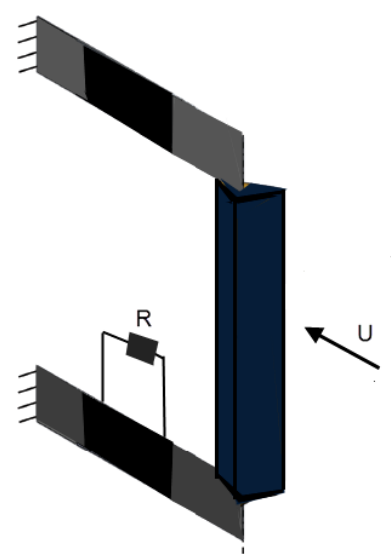

Fig. 23 Galloping energy harvester with tip body having equilateral triangle cross section [79]. (C IOP Publishing. Reproduced with permission. All rights reserved) 


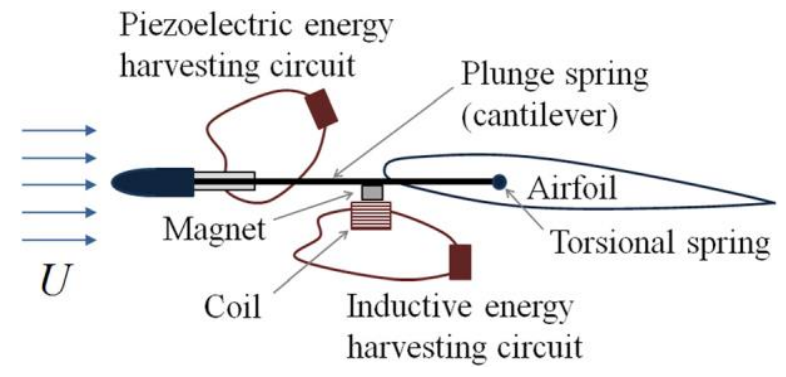

Fig. 24 Schematic of the hybrid piezoelectric-inductive energy harvester [86]. 


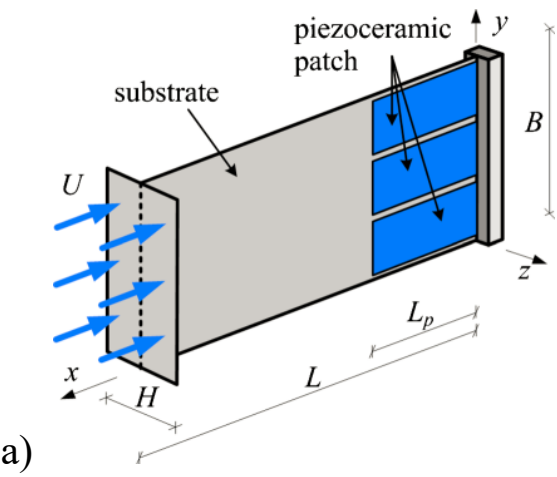

(b)

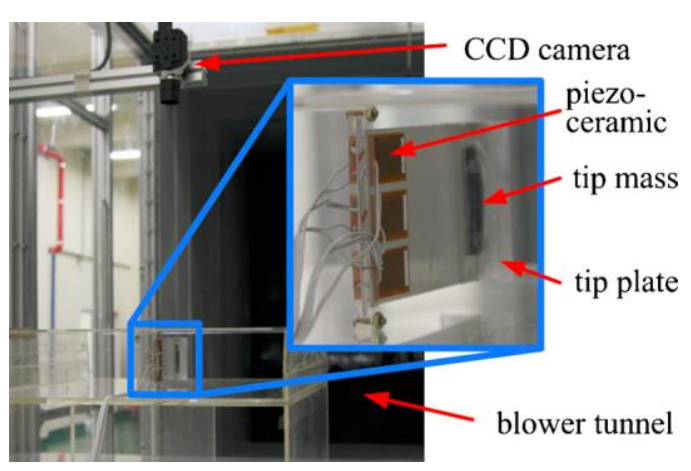

Fig. 25 (a) Schematic of T-shape cantilever energy harvester and (b) experimental setup in the wind tunnel [89]. 
(a)

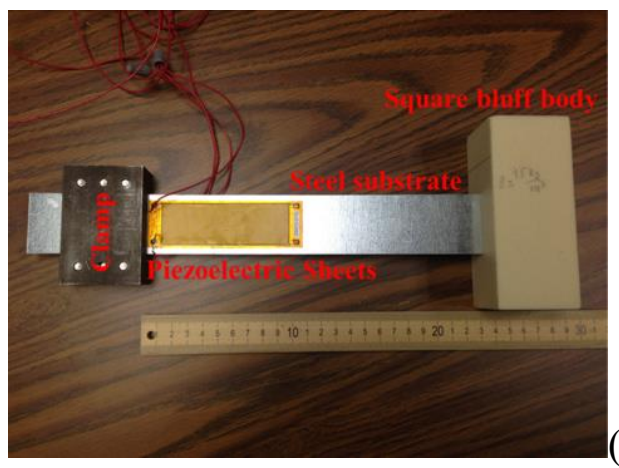

(b)

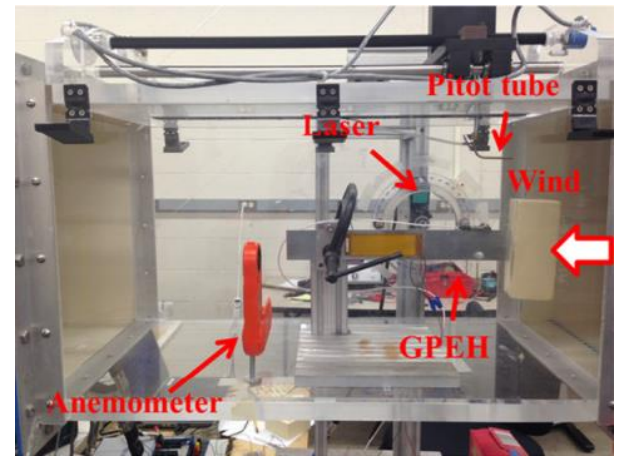

Fig. 26 Galloping piezoelectric energy harvester: (a) prototype, (b) prototype in wind tunnel test section [90]. (C IOP Publishing. Reproduced with permission. All rights reserved) 
(a)

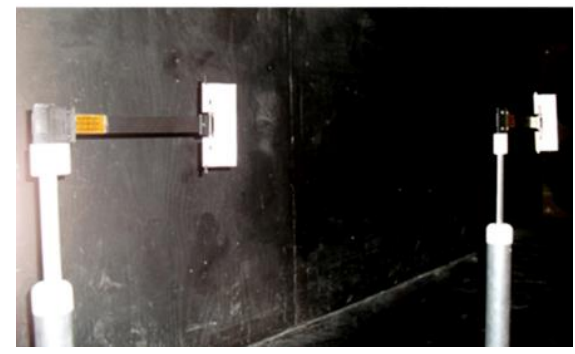

(b)

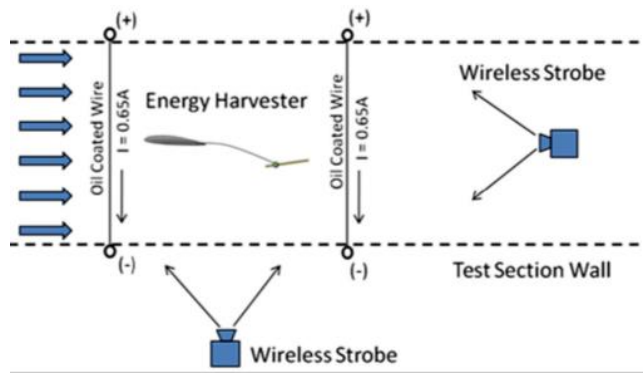

Fig. 27 In (a), two aeroelastic energy harvesters mounted in a wind tunnel test section in tandem configuration; in (b), overhead schematic view of the smoke wire flow visualization setup used to image the wake of the energy harvesters [91]. 
(a)
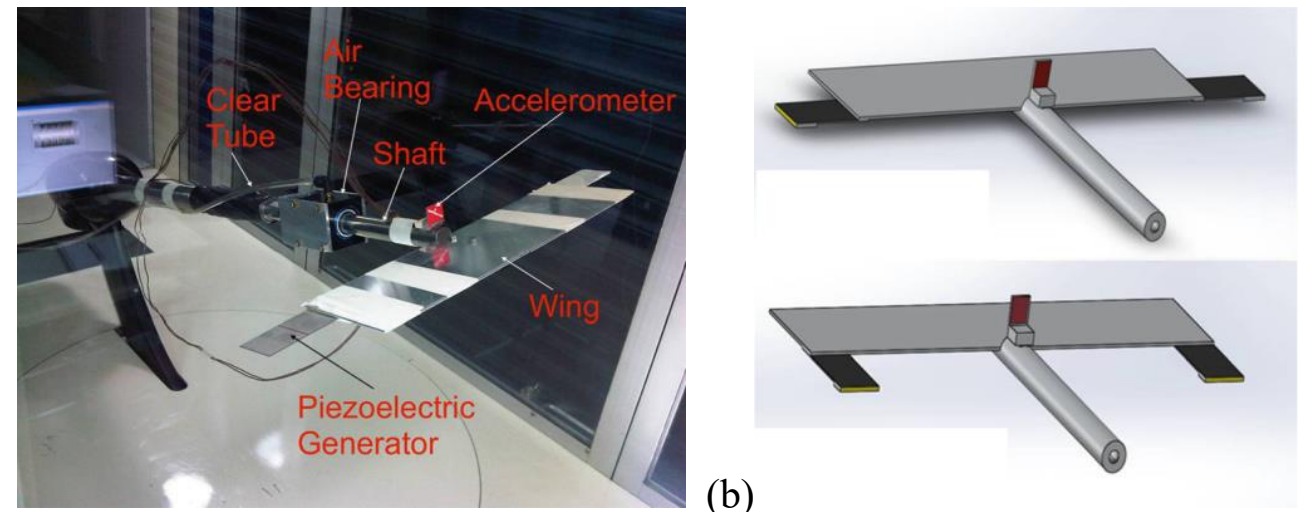

Fig. 28 In (a), experimental setup of an energy harvesting platform in a wind tunnel; and in (b), two configurations of the piezoelectric generators [92]. 
(a)

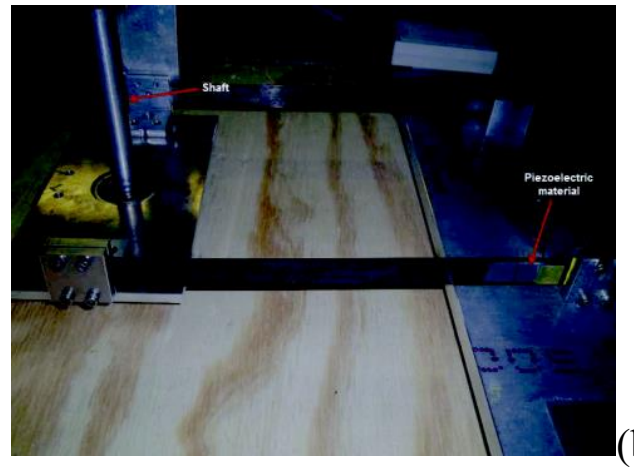

(b)

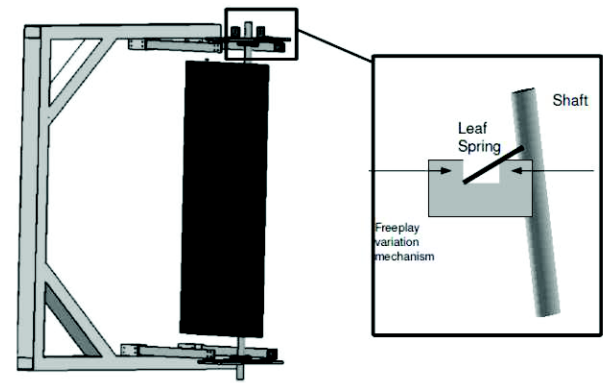

Fig. 29 Schematic of (a) the experimental setup and (b) the pitch free-play mechanism [93]. Reprinted with permission as per http://creativecommons.org/licenses/by-nc-nd/4.0/ 


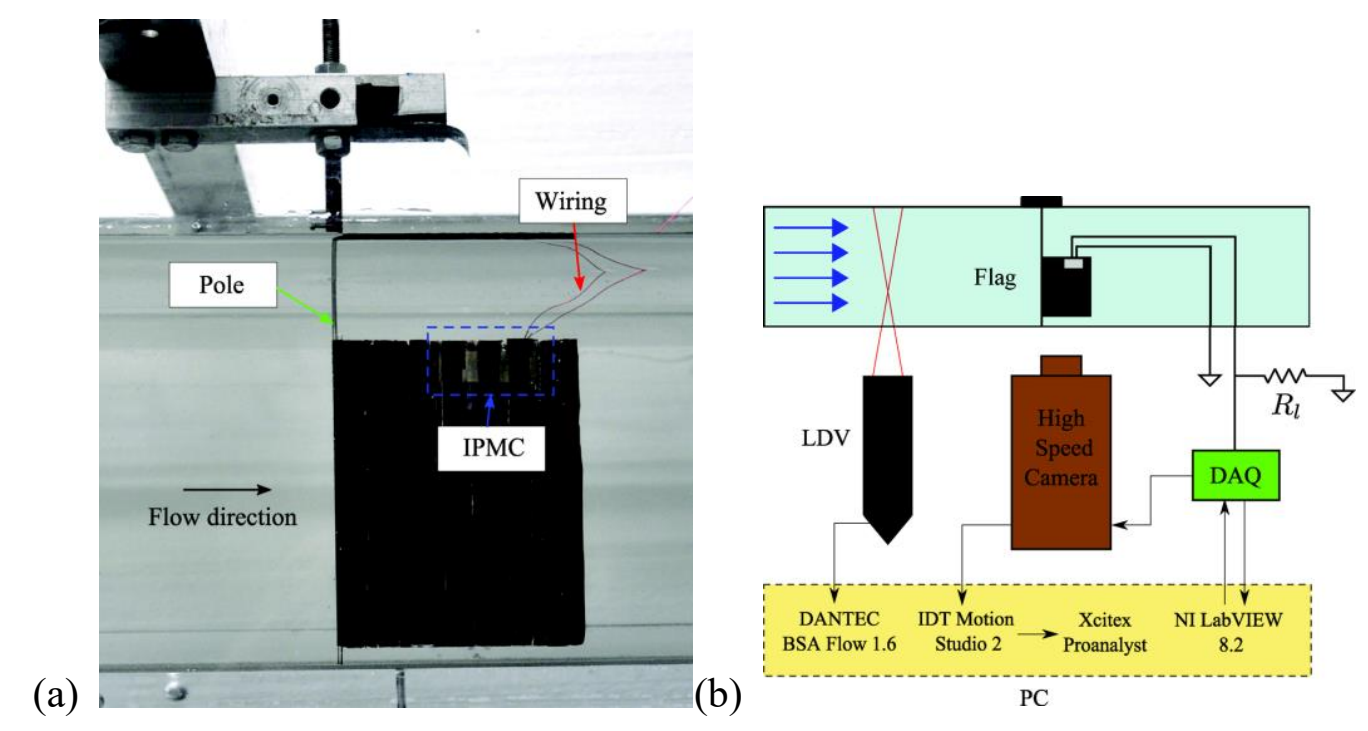

Fig. 30 Schematic of the experimental setup used to measure the harvesting capabilities of an IPMC

mounted on a heavy host flag [94]. IPMC denotes ionic polymer metal composites. 


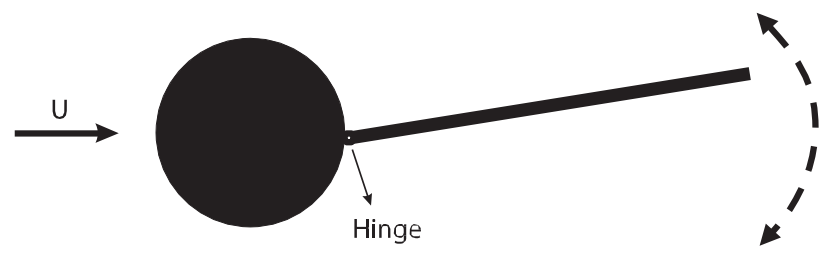

Fig. 31 Schematic of a cylinder with a hinged-rigid splitter plate. The rigid splitter plate was free to rotate about the hinge at the base of the cylinder [95]. 


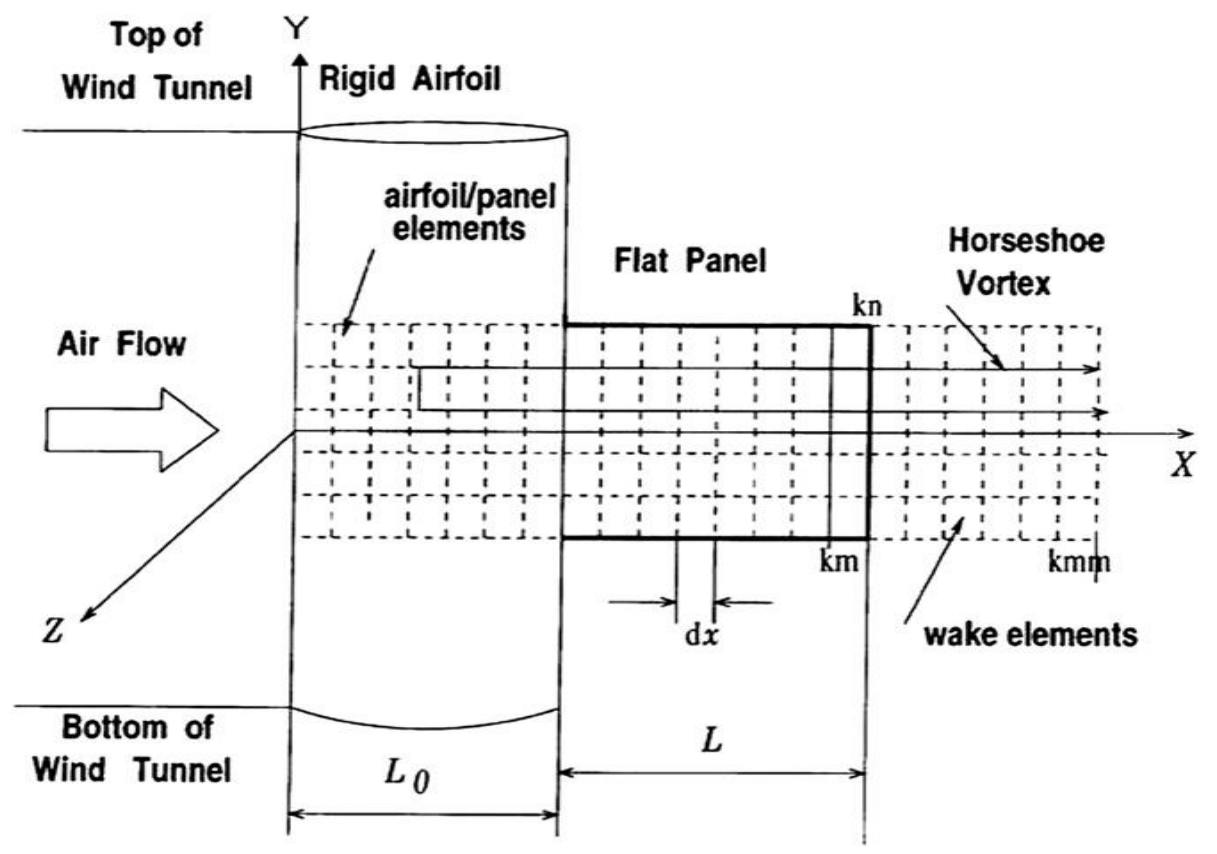

Fig. 32 Experimental setup of plate-based EH in axial flow [57]. 


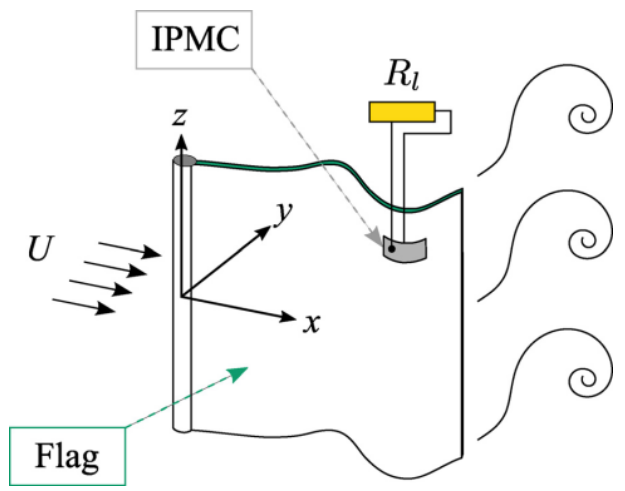

Fig. 33 Schematic of the flexible heavy flag flapping in a uniform water stream comprising the host structure and the ionic polymer metal composites strip [94]. 


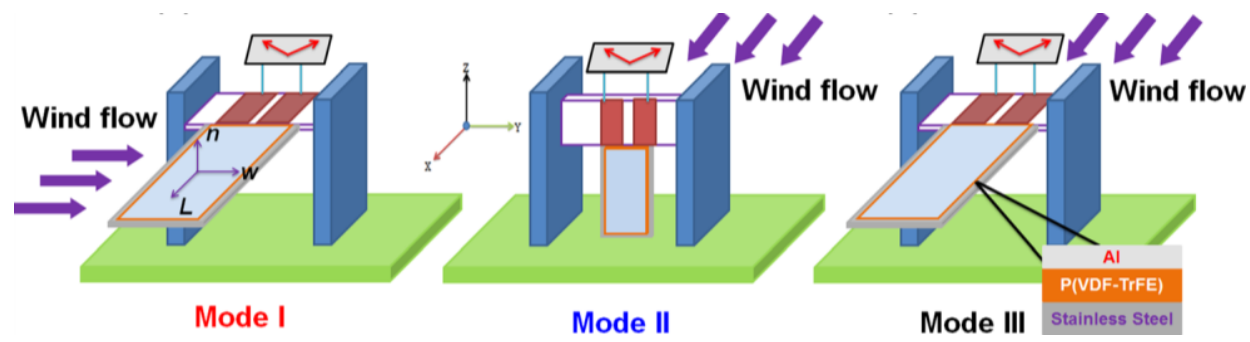

Fig. 34 Schematic of different flapping leaf configurations [30]. 


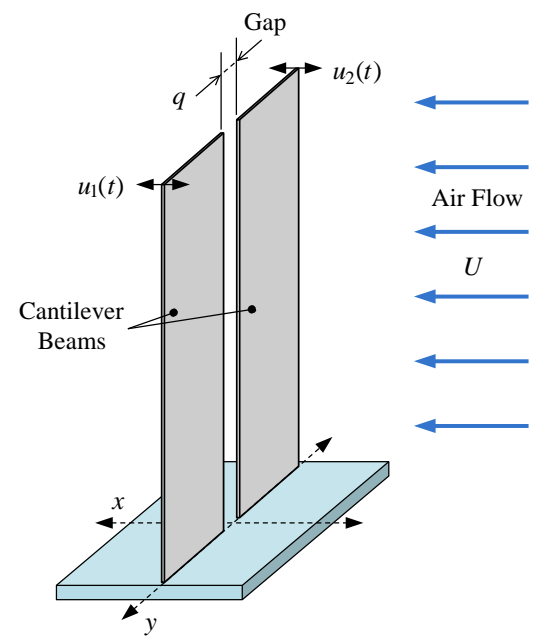

Fig. 35 A schematic of the dual cantilever EH system [100]. 


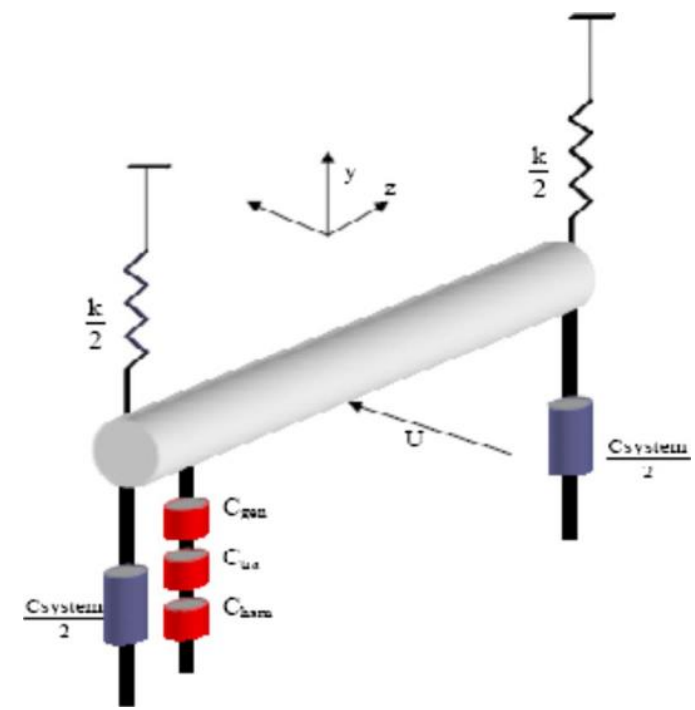

Fig. 36 Schematic of EH from galloping oscillations; from Ref. [101], @ ASME, with permission.

36 


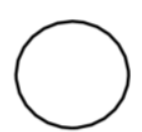

(a) cylinder

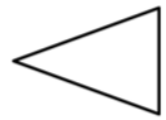

(b) triangle

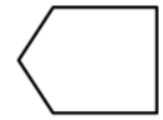

(c) pentagon

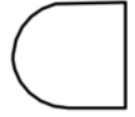

(d) D-shaped

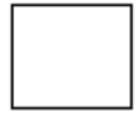

(e) square

Fig. 37 Different cross sections of bluff bodies. 
(a)

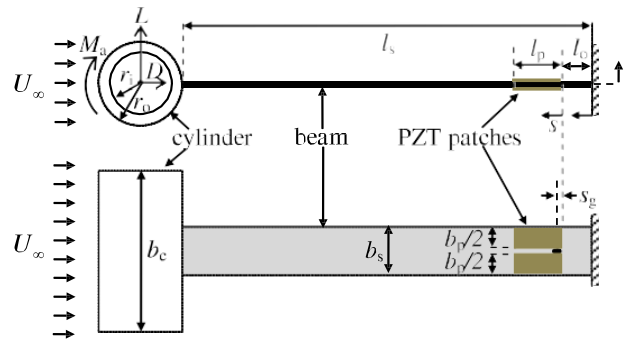

(b)

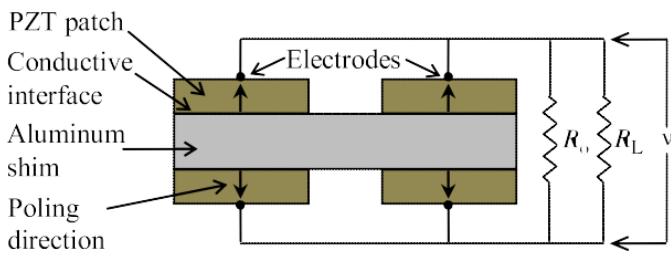

Fig. 38 In (a) top and side views of the self-excited harvester, and in (b) electrode configuration for patches [96]. (@ IOP Publishing. Reproduced with permission. All rights reserved) 
(a)

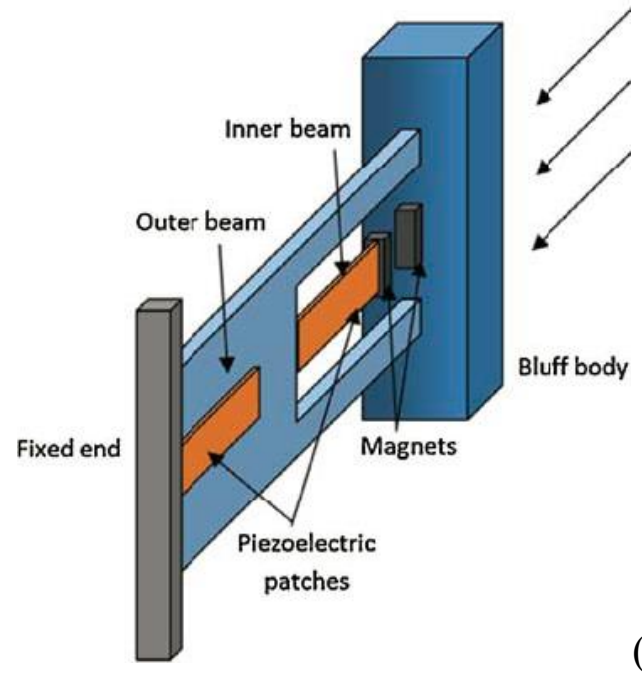

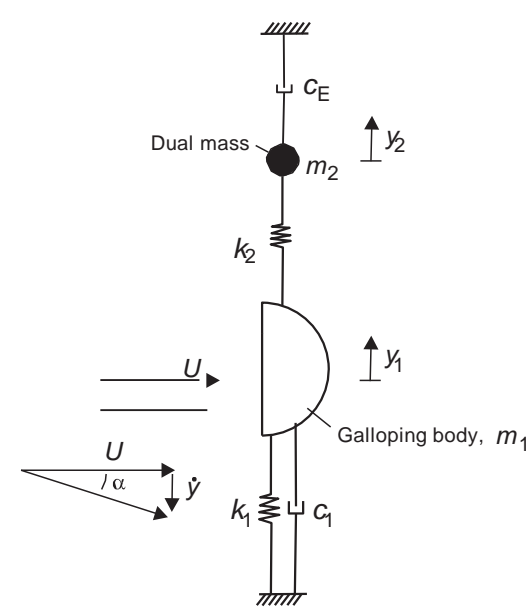

(b)

Fig. 39 Schematics of 2DOF EH from galloping oscillations by (a) Zhao et al. [111] (@ 2014 by The

Japan Society of Applied Physics, reprinted with permission) and (b) Vicente-Ludlam et al. [112]. 


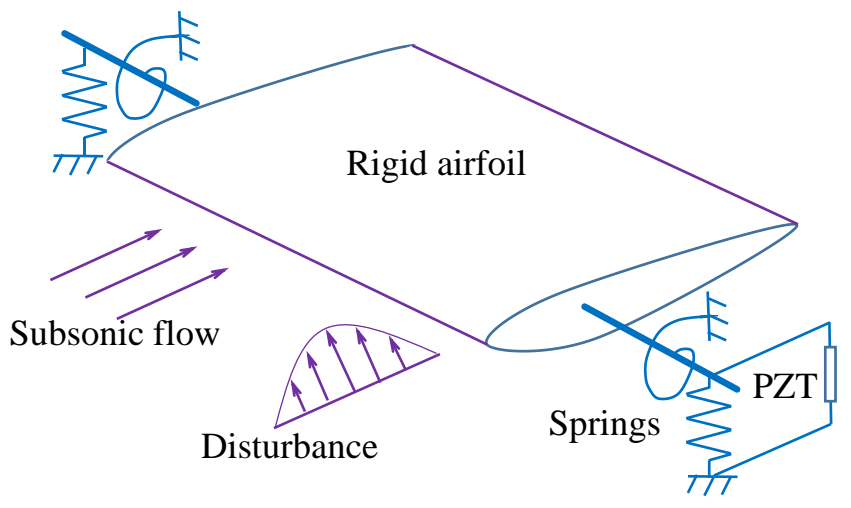

Fig. 40 Schematic of an aerofoil-based EH system [115]. 


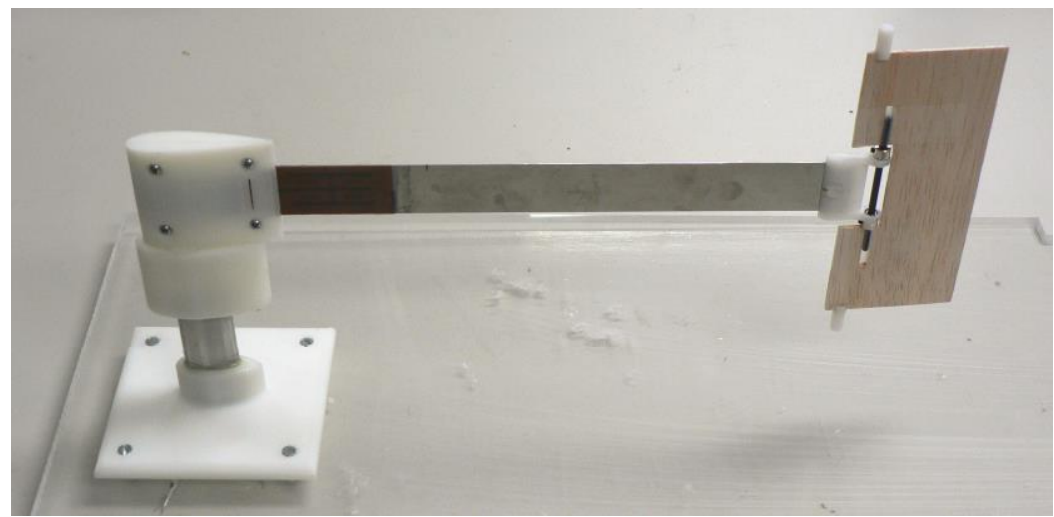

Fig. 41 Aerofoil-based EH; from Ref. [116], @ SPIE, with permission. 


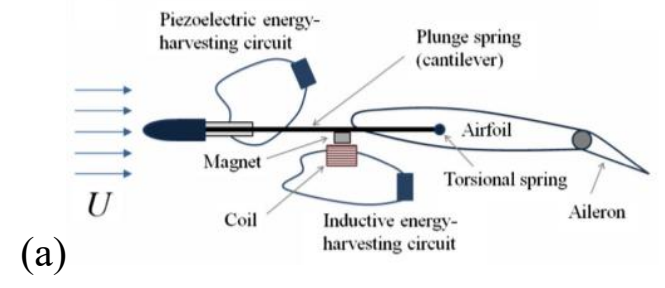

(b)

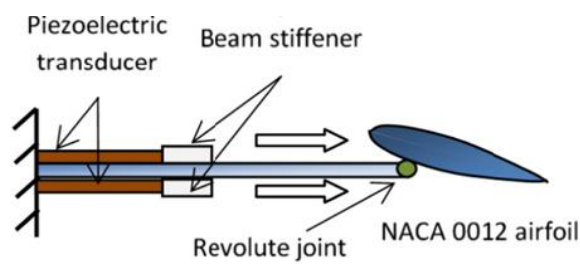

Fig. 42 Schematic of modified aerofoil-based EH devices; in (a) from [87], and in (b) from [130]. (C IOP Publishing. Reproduced with permission. All rights reserved) 


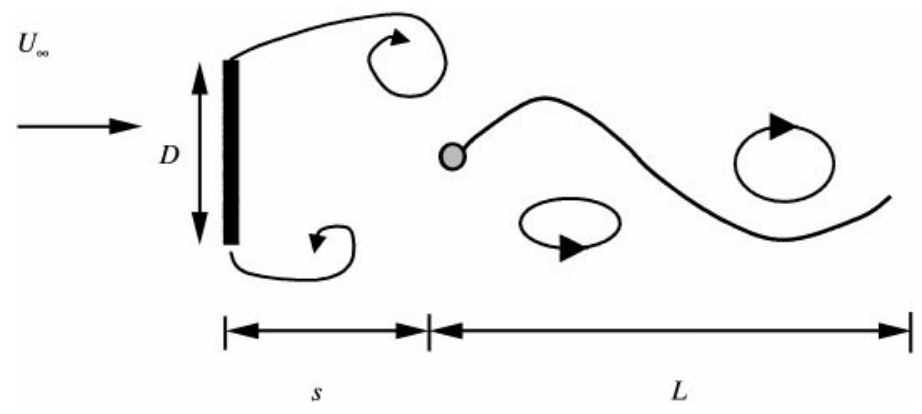

Fig. 43 Schematic of an EH ell [131]. 


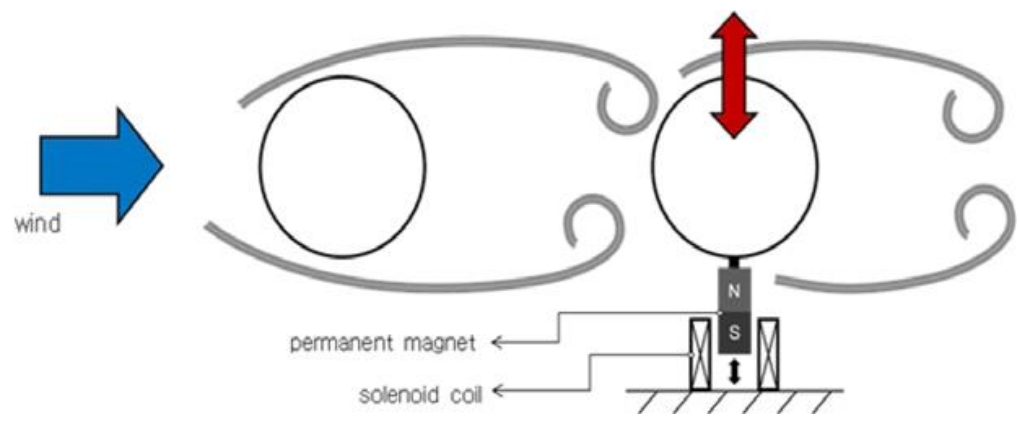

Fig. 44 Schematic of EH from wake galloping phenomena [41]. (C IOP Publishing. Reproduced with permission. All rights reserved) 


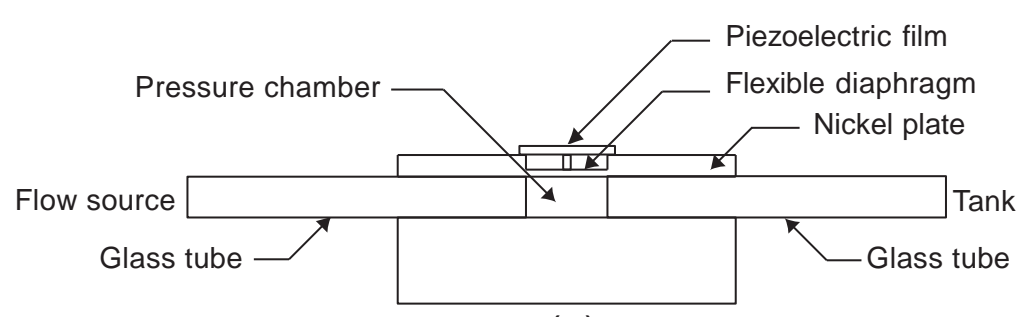

Fig. 45 Schematic of EH based on variable flow via a membrane [142]. 


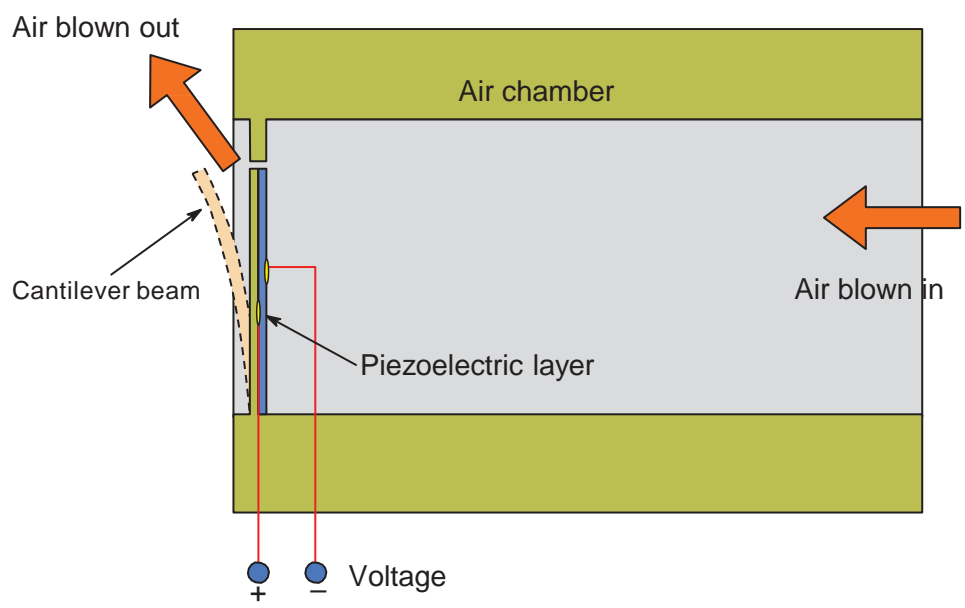

Fig. 46 Schematic of EH based on blown airflow within a cavity [146]. 


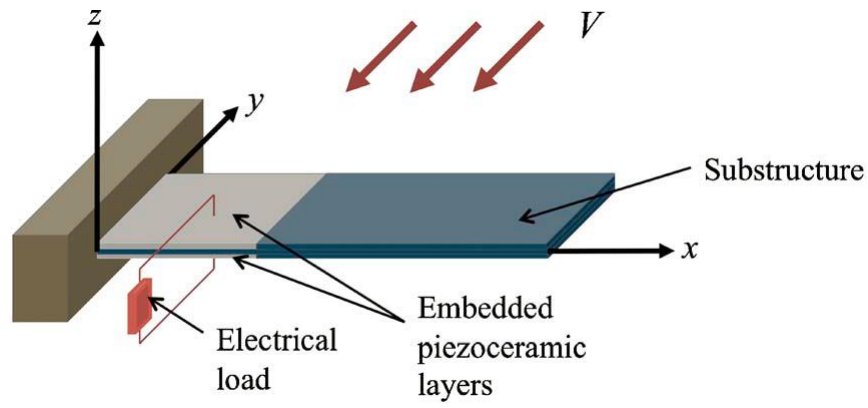

Fig. 47 Schematic of a wing-like plate; from Ref. [152], @ ASME, with permission. 


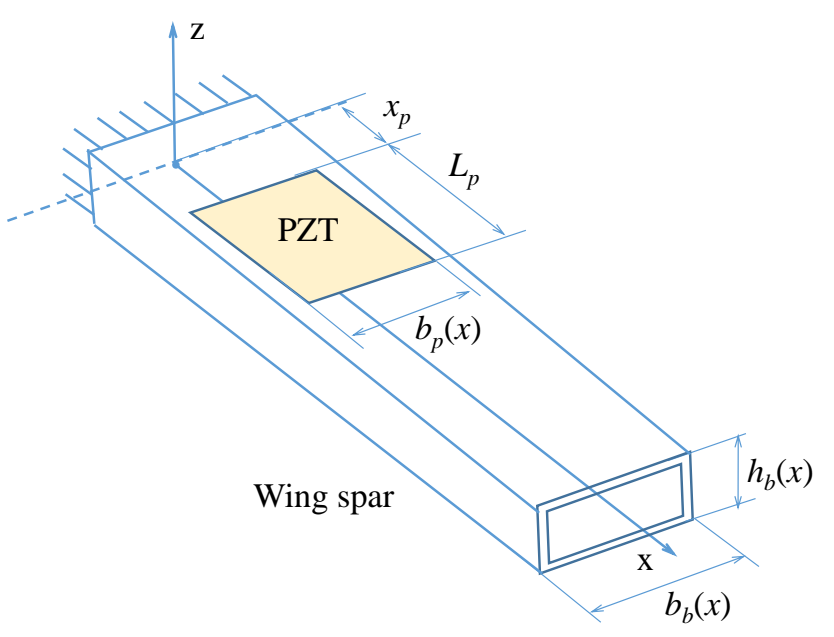

Fig. 48 Schematic of the piezoelectric wing spar [53]. 


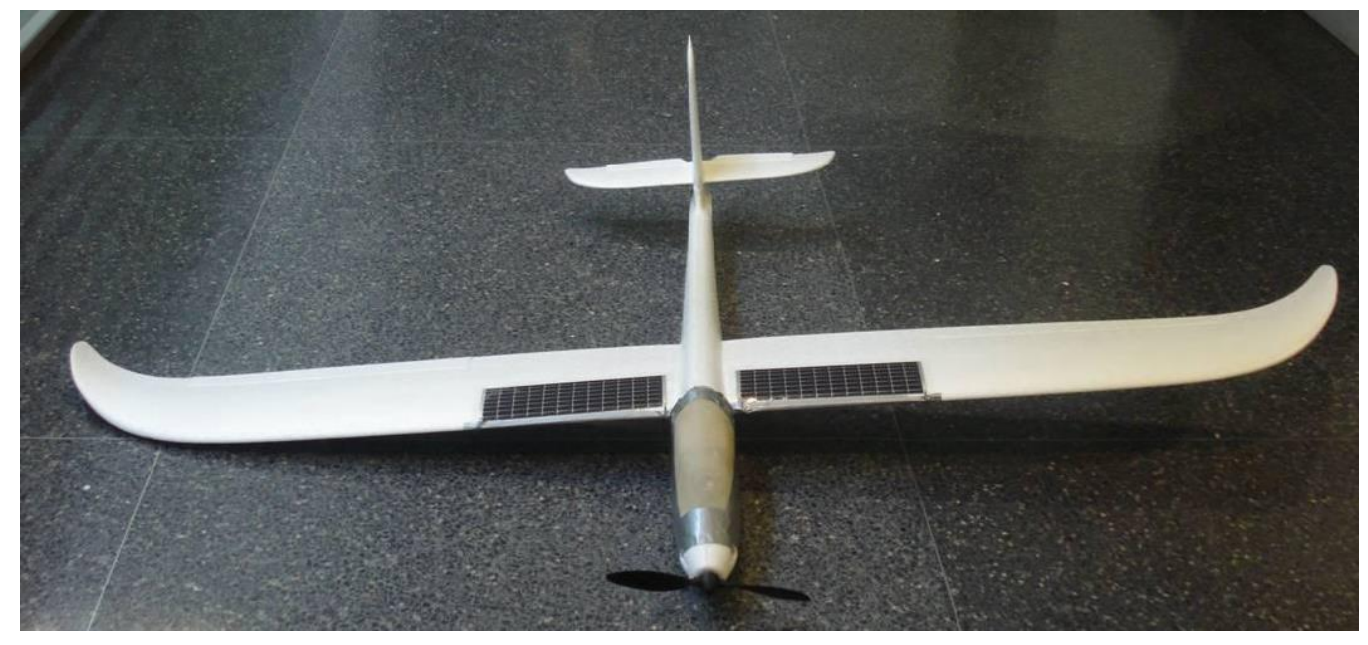

Fig. 49 Remote control aircraft with modifications; from [159], () SPIE, with permission. 


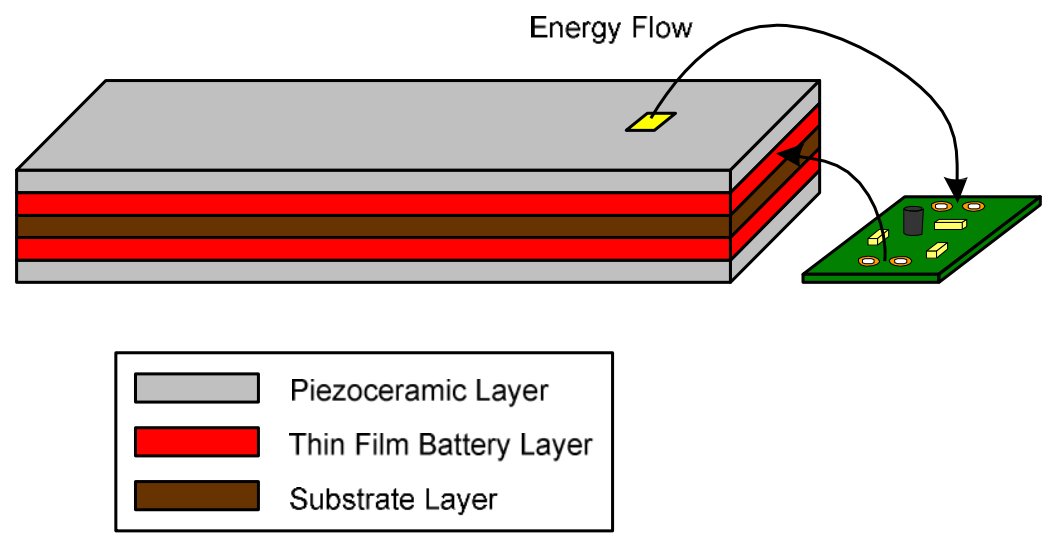

Fig. 50 Schematic of the multifunctional wing spar; from Ref. [161], @ ASME, with permission. 


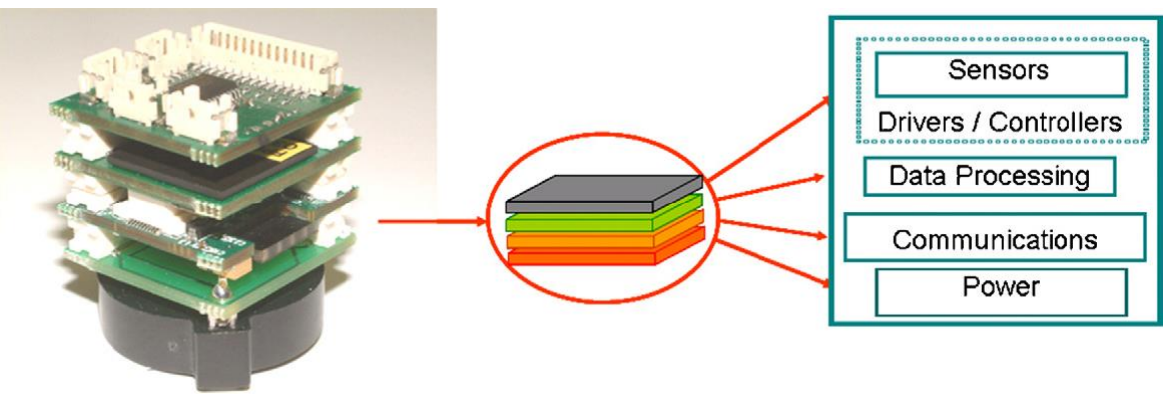

Fig. 51 Photograph and schematic of Tyndall $25 \mathrm{~mm}$ wireless sensor module [168]. 


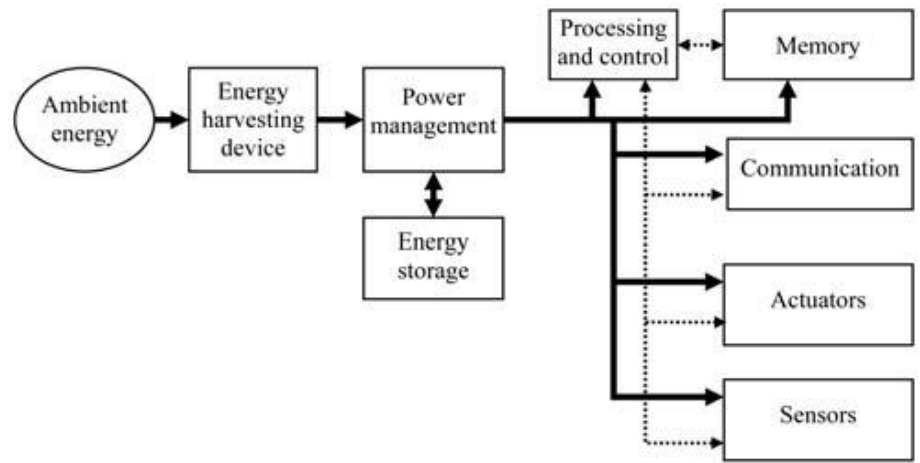

Fig. 52 Generic WSN node with EH devices; from Ref. [167], (c) Springer, with permission. 


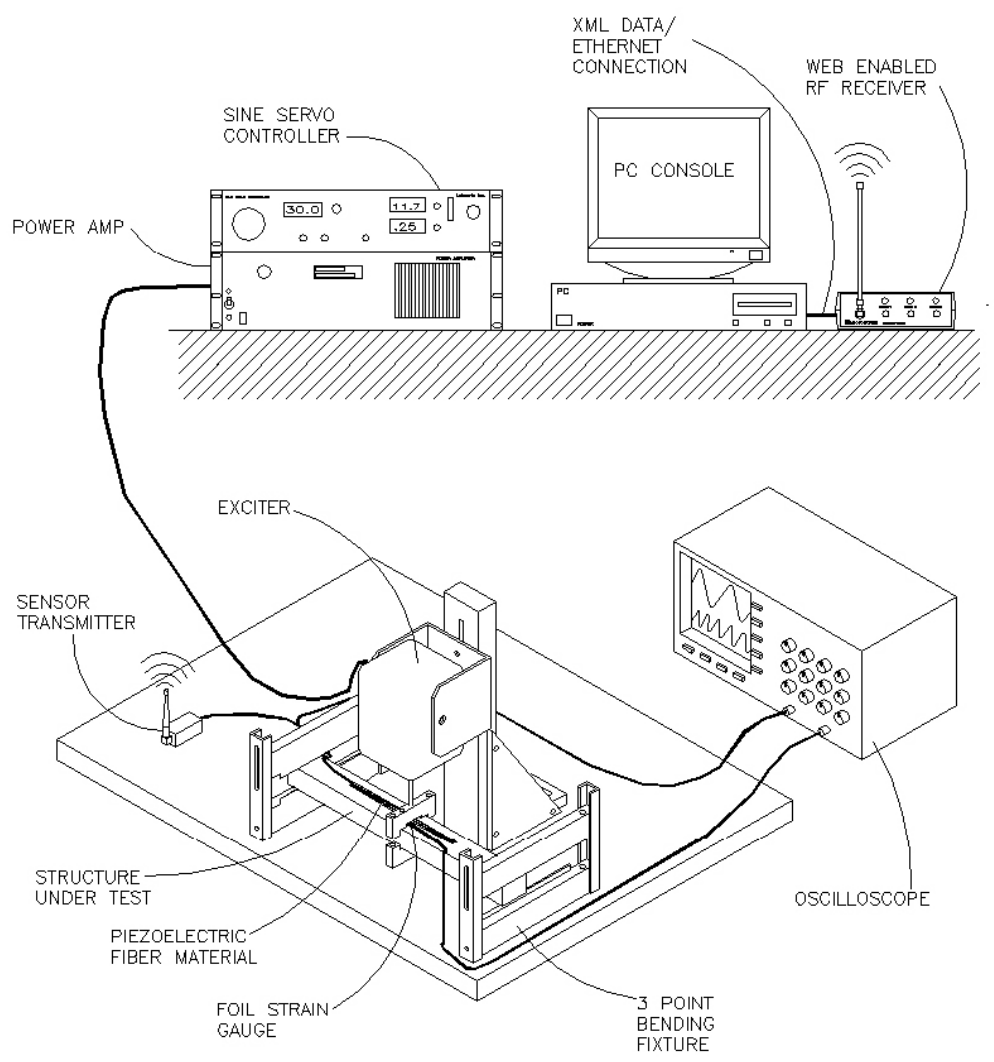

Fig. 53 Schematic of strain EH system; from Ref. [173], @ SPIE, with permission. 


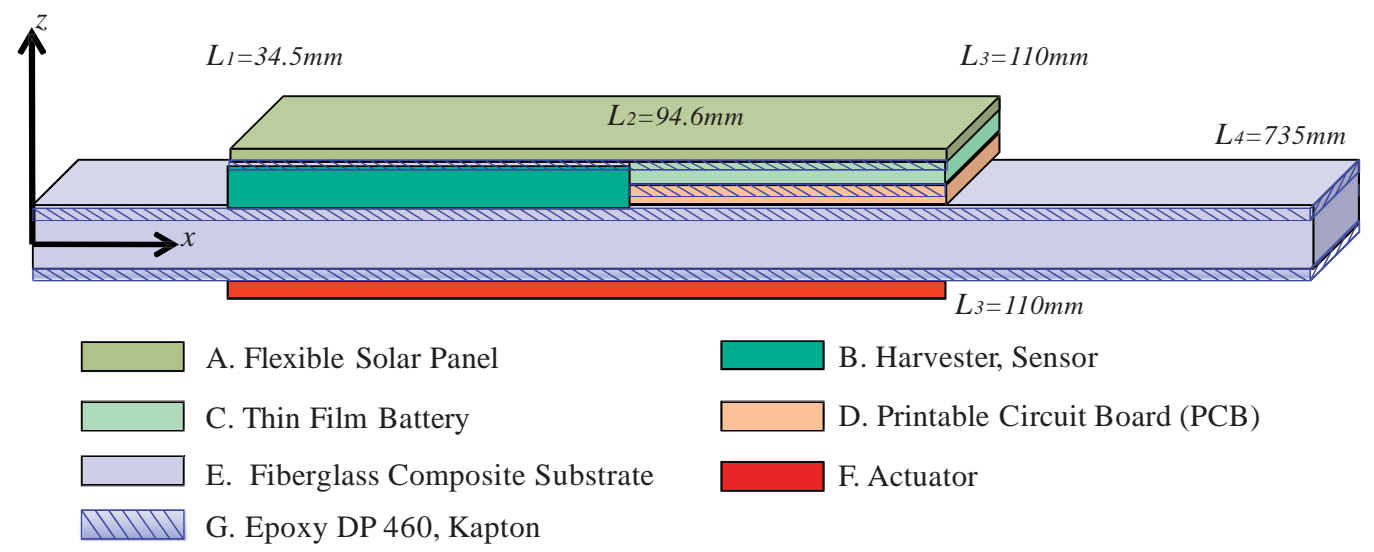

Fig. 54 Concept of multifunctional composite wing spar [183]. 


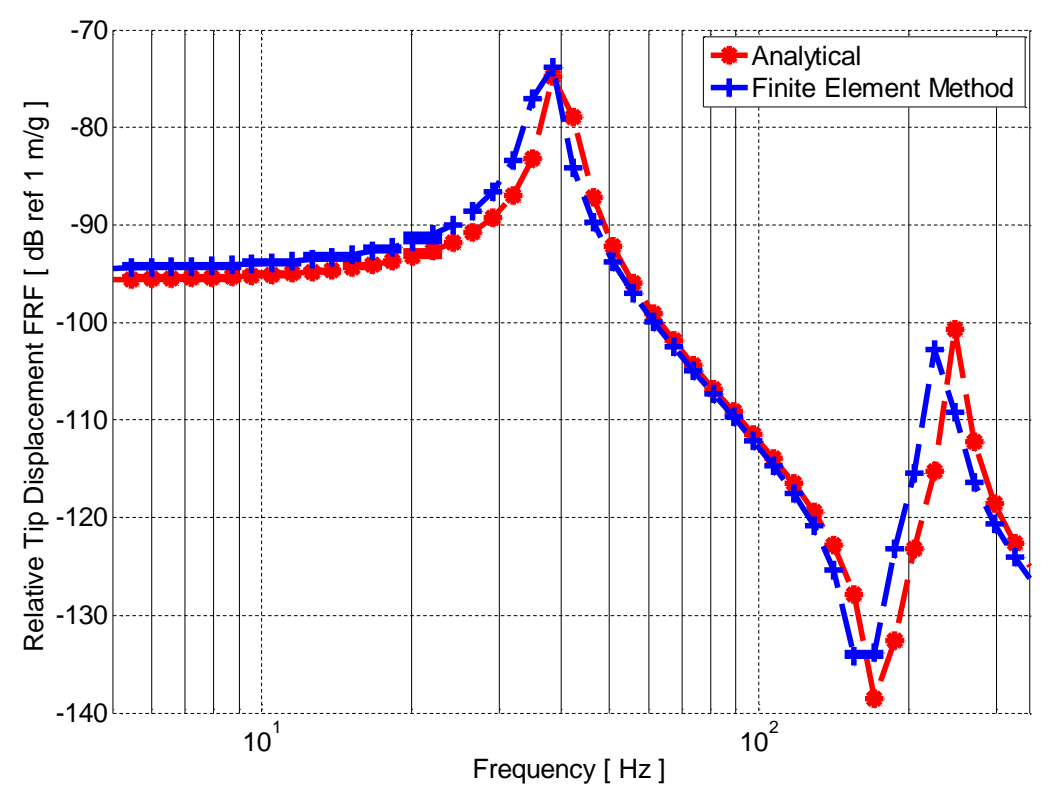

(a)

(b)

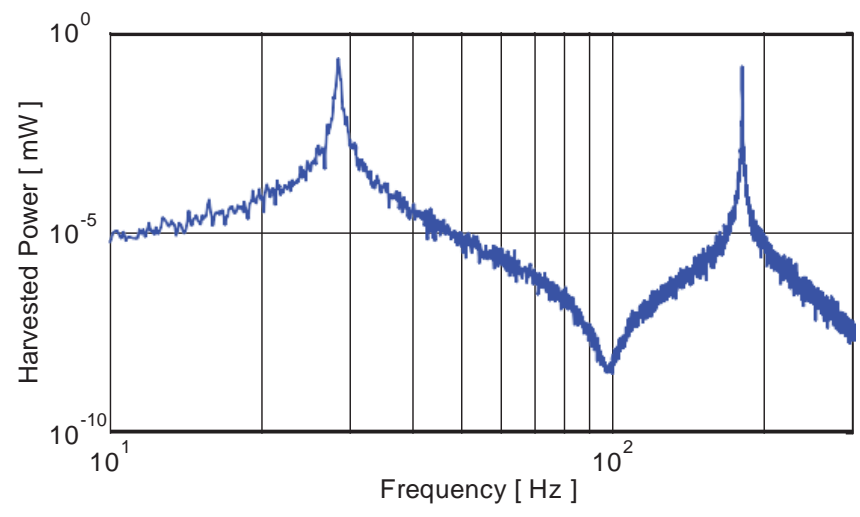

Fig. 55 In (a), dimensionless tip displacement frequency response function of multifunctional composite wing spar, and in (b) harvested power spectrum at one optimal load resistance identified in [183]. 


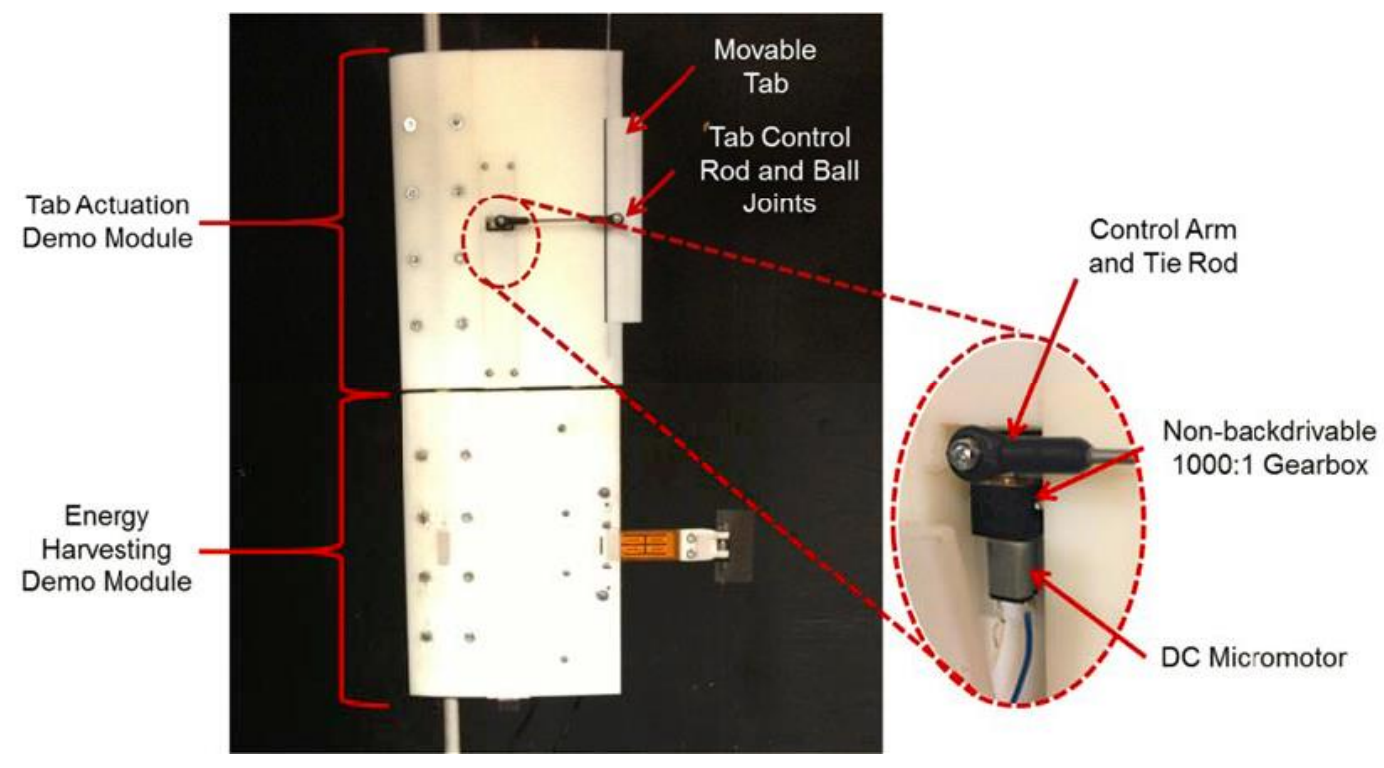

Fig. 56 Wind tunnel apparatus for demonstrating tab actuation powered by aerofoil-based harvester; from

Ref. [184], ( ) SPIE, with permission. 


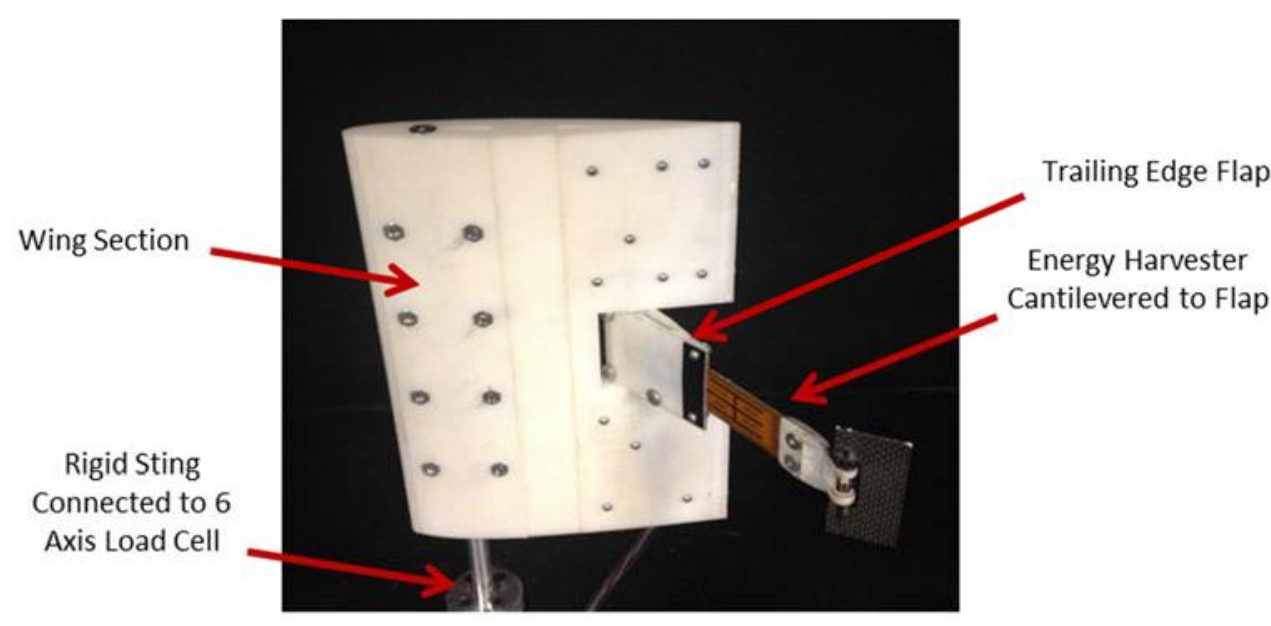

Fig. 57 Apparatus for placing the aeroelastic energy harvester at an angle of attack in the flow; from Ref.

[184], () SPIE, with permission. 